\title{
Combining Conventional Tests and Terminal Restriction Fragment Analysis to Evaluate Microbial Quality of Raw Milk
}

\author{
A thesis \\ Presented to the faculty of \\ California Polytechnic State University \\ San Luis Obispo
}

In Partial Fulfillment

of the requirements for the degree of

Master of Science in Agriculture

\author{
by \\ Haibin Guo \\ January 2011
}


Copyright $\odot 2011$

Haibin Guo

ALL RIGHTS RESERVED 


\section{Approval page}

TITLE: Combining conventional tests and terminal restriction fragment analysis to evaluate microbial quality of raw milk

AUTHOR: $\quad$ Haibin Guo

DATE SUBMITTED: January, 2011

COMMITTEE CHAIR: $\quad$ Dr. Rafael Jimenez-Flores

COMMITTEE MEMBER: $\quad$ Dr. Marie Yeung

COMMITTEE MEMBER: Dr. Chris Kitts 


\section{Abstract \\ Combining Conventional Tests and Terminal Restriction Fragment Analysis to Evaluate Microbial Quality of Raw Milk}

\section{Haibin Guo}

The dairy industry is an important part in the domestic economy in the U.S. and the quality of dairy products hinges on raw milk quality. Microorganisms play a critical role in raw milk quality and they are currently tested and monitored by conventional microbiological tests. Some of the most common conventional tests include somatic cell count (SCC), standard plate count (SPC), coliform count (CC), lab pasteurized count (LPC) and proteolytic strain count (PSC). However, these methods do not correlate with each other or with the quality of milk and milk products. One factor that contributes to this lack of correlation is the insufficient knowledge of microbial communities in raw milk. In this work we aimed to evaluate modern molecular methods to complement traditional quality procedures that may eventually complement conventional tests and improve milk quality evaluation. Therefore, a molecular method, Terminal Restriction Fragment (TRF) analysis was introduced. TRF analysis has been widely used as a tool to investigate the microbial communities in environmental samples. In this study, it was applied to investigate the microbial communities in raw milk and evaluate raw milk quality.

Milk samples were collected for over six months in the Cal Poly dairy farm and evaluated by conventional tests and TRF analysis. Samples were defined as "high quality" milk and "low quality" milk according to each conventional test first. The cutoffs applied were: 50,000 cfu/ml for SPC, 70,000 cells $/ \mathrm{ml}$ for SCC, $100 \mathrm{cfu} / \mathrm{ml}$ for CC and $250 \mathrm{cfu} / \mathrm{ml}$ for LPC. TRF analysis was 
conducted on raw milk samples subsequently. DNA extraction was optimized. Non-Parametric Multivariate Analysis of Variance (NPMANOVA) was applied to TRF profiles from low and high quality milk. The analysis of Similarity of Percentage (SIMPER) was used to determine each TRF peak's contribution to the dissimilarity between the profiles of high and low quality milk. The genus/species represented by TRF peaks were estimated via database matching. In addition, conventional tests and TRF analysis were also used to analyze the factors causing low quality milk. Rain event and cow's apparent health were the two factors investigated since raw milk samples were collected from cows in different apparent health status on wet days and dry days.

Conventional tests revealed strong correlations between the results of SPC and PSC, and SPC and $\mathrm{CC}$ (coefficients of correlation > 0.8). It implied that the results of conventional tests might not be independent, so the statistics based on the assumption of independence of variables were not suitable to analyze the data. SCC showed no strong correlation with any other conventional tests. Raw milk samples were grouped as high quality and low quality according to SPC, $\mathrm{CC}$, SCC and LPC. Using TRF analysis, it was found that there was a significant difference between TRF profiles from low and high quality milk when the quality was defined by SPC or LPC. A TRF peak at 268 bp generated by DpnII was predominant in the TRF profiles and had high abundance in the profiles of low quality milk. Hence, Pseudomonas spp. represented by TRF peak at $268 \mathrm{bp}$ was likely the predominant bacteria in the microbial community associated with raw milk. TRF peaks at 61 bp, 81 bp, 104 bp, 104 bp, 201 bp, 242 bp, 268 bp, and 270 bp contributed the most to the dissimilarity between TRF profiles from different groups of samples. In addition, the presence of DNA derived from viable but non-culturable species that were associated with raw milk quality was detected. 
Rain event was the most important factor affecting the microbial quality of raw milk in this study. Both the conventional tests and TRF analysis showed that there was a significant difference between raw milk samples collected on wet days versus dry days. Samples collected on wet days harbored high bacterial counts and had high abundance of the predominant TRF peaks. In addition, the same TRF peaks contributing the most to the dissimilarity between groups separated by rain event were found to be among those contributing the most to the dissimilarity between groups of high and low quality milk defined by conventional tests. During wet days, the low quality milk was likely caused by the increased dirtiness of cow's teats. Soil microbes are often associated with microorganisms in raw milk such as psychrotrophic bacteria, coliform groups and spore-formers. Cow's apparent health status showed no significant influence on the microbial quality of raw milk.

Overall, the combination of conventional tests and TRF analysis can yield a comprehensive understanding of microbial community in raw milk and improve the evaluation of raw milk quality. TRF analysis was demonstrated as a useful tool and a complement to conventional tests for milk quality evaluation by providing more information on the microbial community associated with raw milk. Findings in this study can offer a basis for further study and may help the dairy industry improve raw milk quality evaluation system. 


\section{Acknowledgments}

I would like to express my gratitude to my advisors, Dr. Rafael Jimenez-Flores, Dr. Marie Yeung, and Dr. Chris Kitts for professional guidance. Without any of you, I could not finish my project and my thesis. Also I would acknowledge the faculty, staff and students in both the Dairy Product Technology Center (DPTC) and Environmental Biotechnology Institute (EBI). I must thank Dr. Phillip Tong, Dr. Joseph Jen and Dr. Yan Yu for providing me with the dual master degree program so I have the opportunity to study at Cal Poly, San Luis Obispo. I would like to thank my parents and my family. They always give me lots of support. Although they are thousands of miles away, their love is still with me every day. 


\section{Table of Contents}

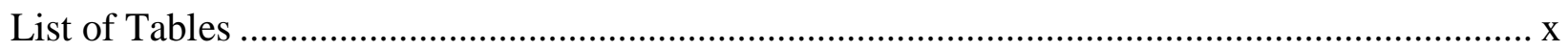

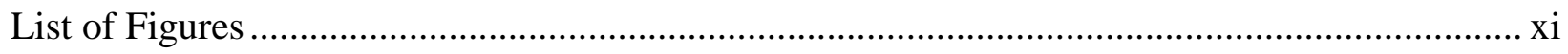

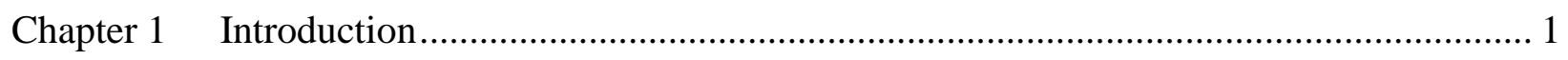

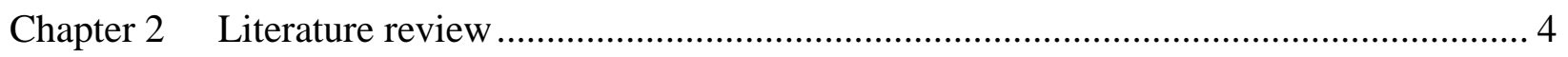

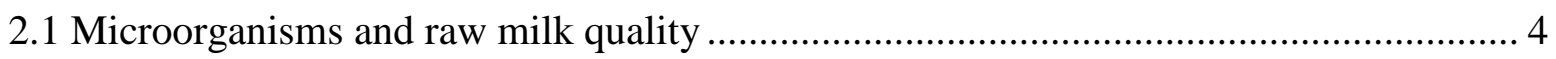

2.1.1 Microflora in raw milk and their effects on raw milk quality.............................. 4

2.1.2 Sources of bacterial contamination in raw milk ................................................. 8

2.1.3 Factors influencing microbial community of raw milk .................................... 9

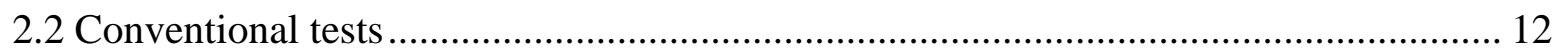

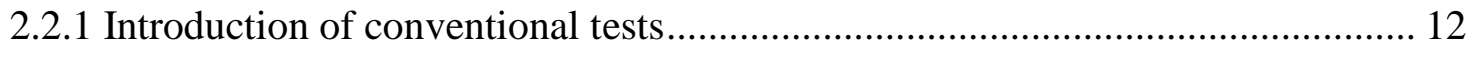

2.2.2 Limitations of conventional microbial tests........................................................ 14

2.3 Terminal restriction fragment (TRF) analysis ......................................................... 15

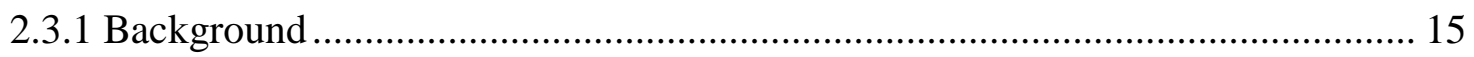

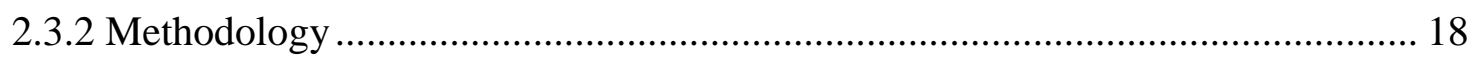

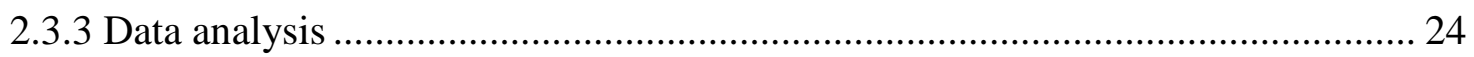

2.3.4 Statistical tools utilized to analyze TRF data.................................................... 27

2.3.5 Potential pitfalls of TRF analysis.................................................................... 30

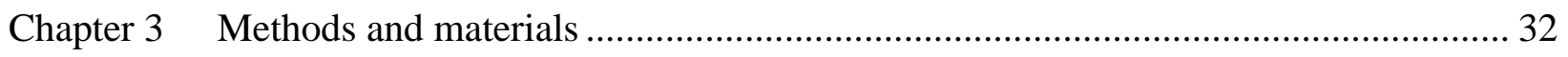

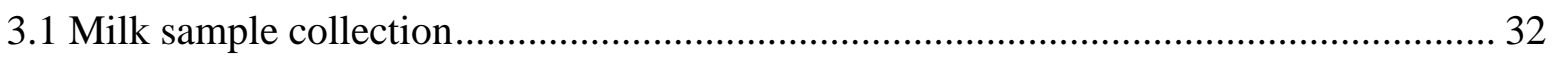

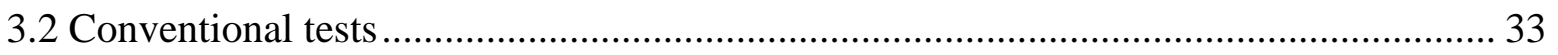

3.3 Milk quality evaluation according to conventional tests ............................................... 35

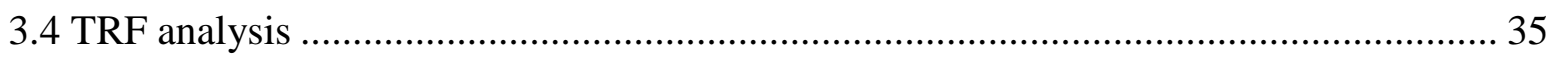




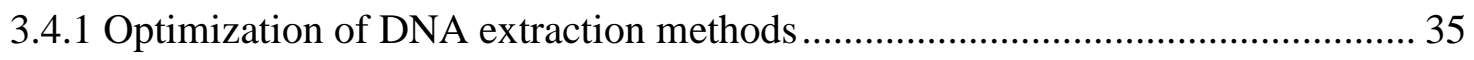

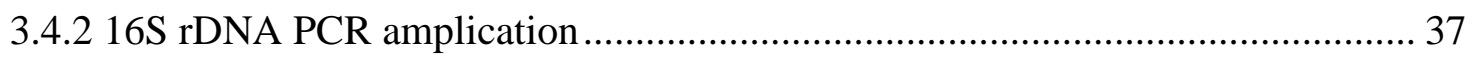

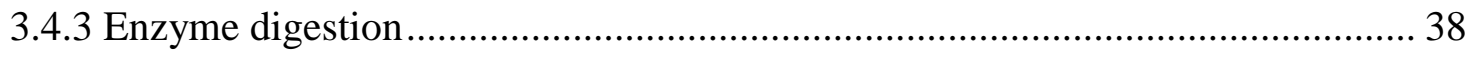

3.4.4 Excess salts removal ............................................................................. 38

3.4.5 Labeled digested fragments. ...................................................................... 38

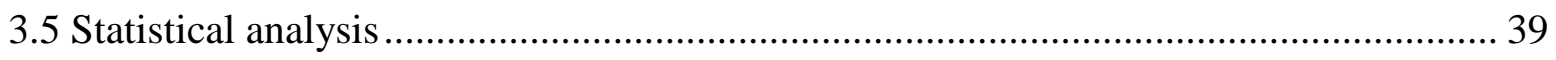

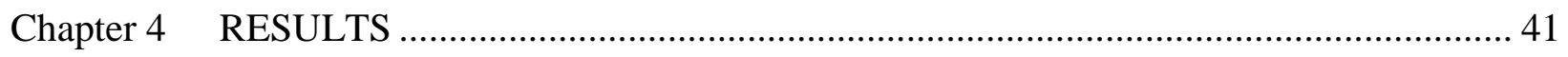

4.1 Optimization of DNA extraction ............................................................................. 41

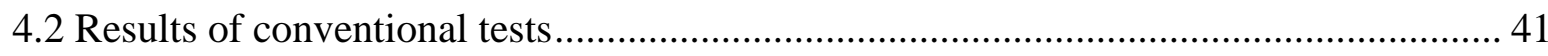

4.3 Raw milk quality evaluation by conventional tests and TRF analysis ........................... 43

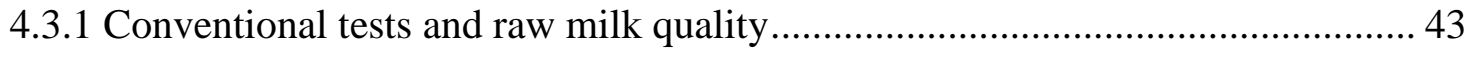

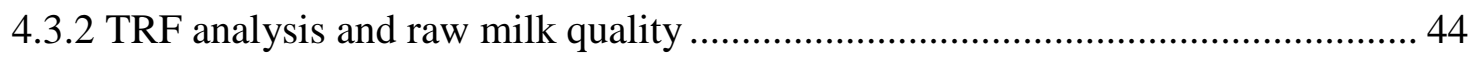

4.4 Factors influencing milk quality …………………................................................... 47

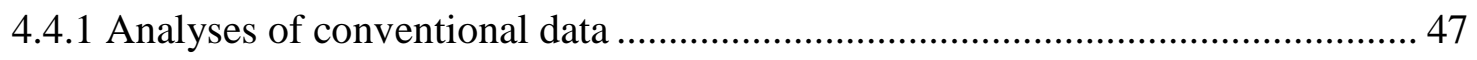

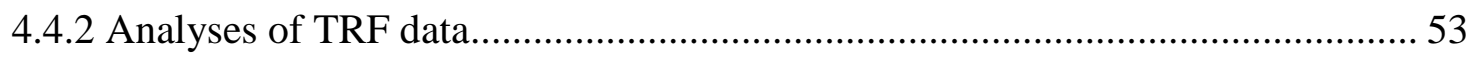

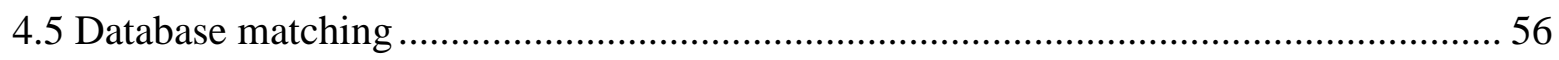

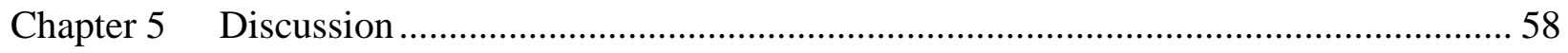

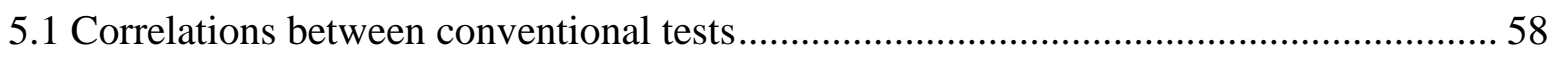

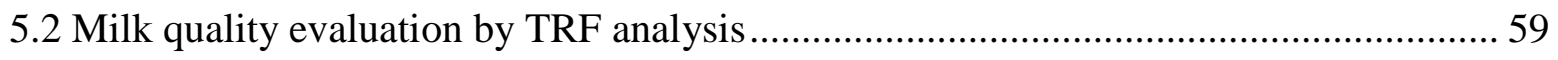

5.3 Complementation between TRF analysis and conventional tests................................... 62

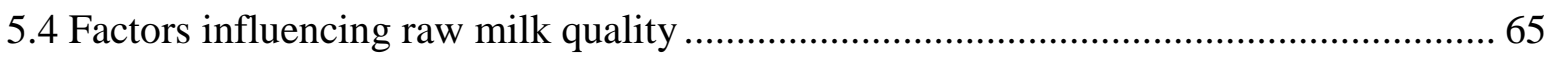

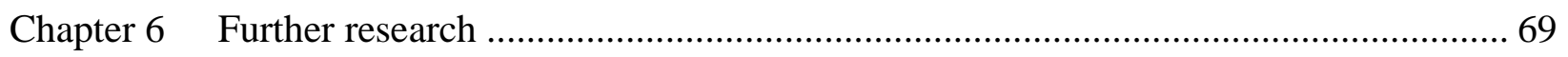

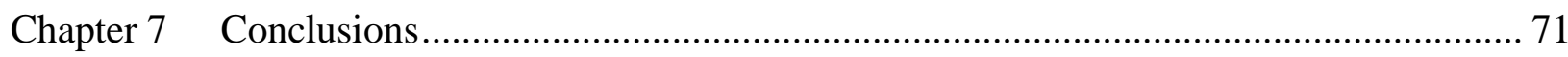

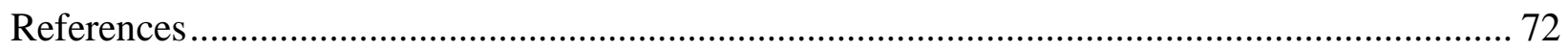

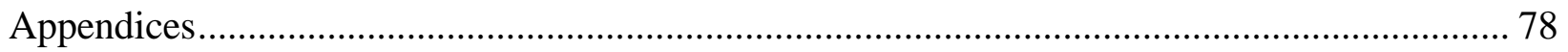




\section{List of Tables}

TABLE 1. SPORE-FORMERS CAUSING SPOILAGE IN FOODS (SCHELDEMAN ET AL., 2006) ............................................

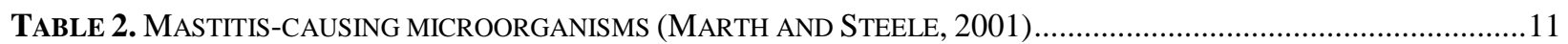

TABLE 3. CHEMICAL, ENZYMATIC AND MECHANICAL APPROACHES FOR CELL LYSIS ……........................................20

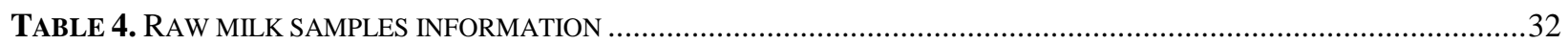

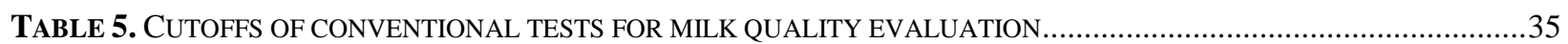

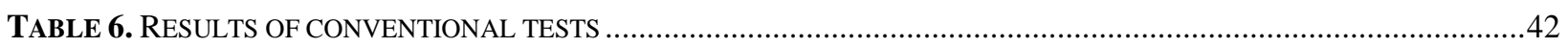

TABLE 7. CORRELATION COEFFICIENTS BETWEEN RESULTS OF DIFFERENT CONVENTIONAL TESTS............................43

TABLE 8. SUMMARY OF MILK QUALITY EVALUATION BY SPC, SCC, CC AND LPC ................................................43

TABLE 9. ANOVA RESULTS OF NUMBERS OF TRF PEAKS (DPNII) .....................................................................4

TABLE 10. FIFTEEN TRF PEAKS CONTRIBUTING THE MOST TO THE DISSIMILARITY BETWEEN HIGH AND LOW

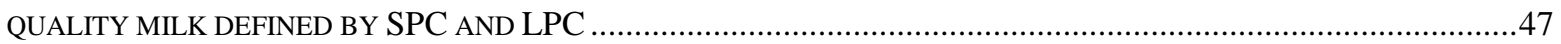

TABLE 11. MANOVA OF CONVENTIONAL TESTS, RAIN EVENT, COW'S APPARENT HEALTH AND INDIVIDUAL COWS. ..52

TABLE 12. P-VALUES FROM ANOVA OF PH, SPC, SCC, CC, LPC AND PSC VERSUS RAIN EVENT, COW'S APPARENT HEALTH STATUS AND INDIVIDUAL COWS (“*” IS LABELED WHERE P-VALUE < 0.01) ...........................52

TABLE 13. OUTPUT OF NPMANOVA FOR TRF DATA BY DPNII VERSUS RAIN EVENT, COW'S APPARENT HEALTH

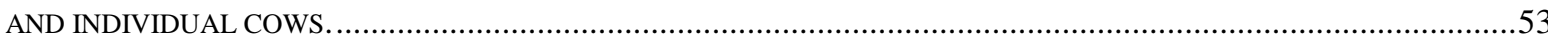

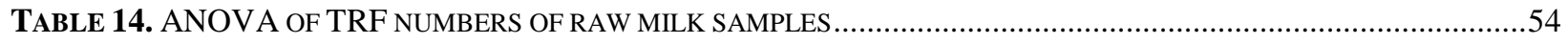

TABLE 15. TRF PEAKS (DPNII) CONTRIBUTING THE MOST TO THE DISSIMILARITY BETWEEN BACTERIAL COMMUNITY ASSOCIATED WITH SAMPLES COLLECTED IN WET AND DRY DAYS ..................................................55

TABLE 16. DATABASE MATCHING OF TRF PEAKS ASSOCIATED WITH RAW MILK QUALITY (BP = BASE PAIR) ...............56 


\section{List of Figures}

FigURE 1. TRF ANALYSIS PROCEDURES (GRUNTZIG ET AL., 2002). 1. DNA EXTRACTION. 2. DNA AMPLIFICATION AND FLUORESCENT LABELING. 3. RESTRICT ENZYME DIGESTION 4. GEL ELECTROPHORESIS. 5. TRF PROFILES...19

FigURE 2. THE DIFFERENCES OF AVERAGE ABUNDANCE OF TRF PEAKS (DPNII) BETWEEN LOW AND HIGH QUALITY MILK DEFINED BY SPC. FOR EACH PEAK, THE DIFFERENCE OF AVERAGE ABUNDANCE $=$ THE AVERAGE ABUNDANCE FROM LOW QUALITY MILK - THE AVERAGE ABUNDANCE FROM HIGH QUALITY MILK.

FigURE 3. THE DIFFERENCES OF AVERAGE ABUNDANCE OF TRF PEAKS (DPNII) BETWEEN LOW AND HIGH QUALITY MILK DEFINED BY LPC. FOR EACH PEAK, THE DIFFERENCE OF AVERAGE ABUNDANCE $=$ THE AVERAGE ABUNDANCE FROM LOW QUALITY MILK - THE AVERAGE ABUNDANCE FROM HIGH QUALITY MILK.

FIGURE 4. SCORE PLOT OF THE 1ST AND 2ND PRINCIPAL COMPONENTS FROM PCA OF CONVENTIONAL TESTS-SAMPLES GROUPED BY RAIN EVENT.

FIGURE 5. SCORE PLOT OF THE 1ST AND 2ND PRINCIPAL COMPONENTS FROM PCA OF CONVENTIONAL TESTS-SAMPLES GROUPED BY COW'S APPARENT HEALTH

FIGURE 6. BIPLOT OF THE $1^{\text {ST }}$ AND $2^{\text {ND }}$ PRINCIPAL COMPONENT FROM PCA OF CONVENTIONAL TESTS ..........................49

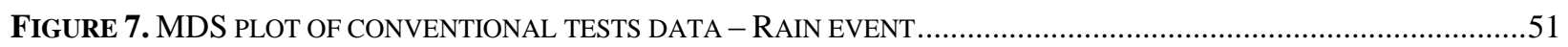

FIGURE 8. MDS PLOT OF CONVENTIONAL TESTS DATA - COW'S APPARENT HEALTH ..................................................51

FIGURE 9. THE DIFFERENCES OF AVERAGE ABUNDANCE OF TRF PEAKS (DPNII) BETWEEN MILK SAMPLES COLLECTED ON WET AND DRY DAYS. FOR EACH PEAK, THE DIFFERENCE OF AVERAGE ABUNDANCE $=$ THE AVERAGE ABUNDANCE FROM MILK SAMPLES COLLECTED ON WET DAYS - THE AVERAGE ABUNDANCE FROM MILK SAMPLES COLLECTED FROM DRY DAYS. 


\section{Chapter 1 Introduction}

The U.S. dairy industry, which has a hundred-billion dollar market, is an important part of the domestic economy. Although each kind of dairy product in the market has its own standard of identity in terms of chemical, physical and microbial criteria, the overall quality often hinges on the quality of raw milk, the common source of all products. The presence of microorganisms and their metabolite products play a major role in raw milk quality, and can extend to pasteurized products. For example, bacteria in raw milk can produce proteases and lipases to cause spoilage by hydrolyzing milk proteins and lipids respectively. Moreover, due to the heat stability of some enzymes and heat resistance of psychrotrophic gram-positive bacteria such as Bacillus, a high number of microorganisms often leads to poor quality of pasteurized milk and other final products, despite the fact that pasteurization destroys a significant amount of these cells and inactivates the enzymes (Barbano et al., 2006).

Total bacteria count and somatic cell count are standard assays used in the pasteurized milk ordinance (PMO) to evaluate raw milk quality. Other tests such as coliform count (CC), lab pasteurized count (LPC), etc. are also adopted by dairy farmers to detect microorganisms in raw milk. Although the criteria are widely accepted in the dairy industry, the conventional tests have limitations. First, there is no clear understanding of the composition of microbial communities in raw milk in terms of the genus/species and their abundance (Lafarge et al., 2004). Second, conventional methods cannot detect the microorganisms that are viable but non-culturable on the culture media for conventional tests (Wegmuller et al., 1993). 
Consequently, no consensus has been reached on what combination of tests can produce results with the most relevance in assessing raw milk quality under current milk production and processing conditions. Due to the limitations of conventional microbial tests, it is essential to introduce more advanced technologies to understand the microbial communities of raw milk with the ultimate goal of enhancing milk quality.

To this end, molecular methods appear to be a good option due to properties to enhance the study of complex microbial communities. These methods are usually culture-independent, and produce a pattern or profile of DNA amplified from a sample reflecting the microbial community structure. TRF analysis is one of these methods that has been widely applied in the microbial ecology study of soil, feces, water, etc (Kitts, 2001). Compared to DNA sequencing, TRF analysis has the advantage of producing results more rapidly. In addition, TRF data is automatically digitalized and therefore more conveniently analyzed with a variety of multivariate statistical tools (Tanaka et al., 2010).

Multivariate analyses are useful and essential tools for analysis of TRF data. Initially, principal component analysis (PCA) was widely used. However, TRF data is not normally distributed, and PCA techniques may fail to produce a meaningful analysis. In addition, it is still questionable whether a transformation of data (such as logarithmic) can overcome this problem. Based on these limitations, more robust non-parametric methods, such as multidimensional scaling plot (MDS) and analysis of similarity (ANOSIM), have been proposed. These two methods overcome the problem of non-normally distributed data, and they are also capable of showing the existence of statistically significant differences between samples (Clarke, 1993). Nevertheless, the main drawback of these two methods is the limitation of analyzing data with more than one factor, since the variation cannot be 
partitioned. In this study, rain event and cow's apparent health status were involved in TRF analysis. Thus, an improved non-parametric multivariate analysis method from Anderson (2001) was employed because it was capable of partitioning the variation. Using this method, the influence of each factor and their interaction were revealed.

The overall goal of this thesis is to study and evaluate combinations of the conventional methods and TRF analysis in order to improve raw milk quality evaluation. Besides the information from conventional methods, TRF analysis is expected to provide more information on the structure of the microbial community and reveal how the microbial community in raw milk is influenced by rain event and cow's apparent health status. 


\section{Chapter 2 Literature review}

\subsection{Microorganisms and raw milk quality}

Milk is a good source of nutrients for human beings (Boor et al., 1998). It provides protein, fat, lactose, vitamin and mineral combined with neutral $\mathrm{pH}$ and in the mean time, it also serves as a good medium for the growth of various microorganisms. However, a large number of microorganisms in raw milk can hydrolyze milk components (e.g. fat and protein), change milk $\mathrm{pH}$, create negative-sensory compounds and adversely affect the quality of pasteurized milk and other milk products such as yoghurt and cheeses. This section will discuss various types of microorganisms in raw milk and their effects on raw milk quality, the sources of microbial contamination and factors that can influence the microbial community in raw milk.

\subsubsection{Microflora in raw milk and their effects on raw milk quality}

Numerous microorganisms can be found in raw milk and have been reported to play an important role in raw milk quality (Boor et al., 1998). Microorganisms consume nutritional components of milk to propagate and maintain their viability. Milk components, including protein, fat and lactose are degraded by the microorganisms to yield products that contribute to undesirable texture, flavor and taste. Finally, microorganisms can cause spoilage and shorten the shelf life of milk and milk products (Marth and Steele, 2001).

Generally, raw milk is refrigerated at $4{ }^{\circ} \mathrm{C}$ immediately following milking, during storage on the farm and transportation to processing plants. Since raw milk is kept cold, the growth of psychrophilic and psychrotrophic microorganisms is selected while the growth of mesophilic and 
thermophilic microorganisms is suppressed. Therefore, the former group is often regarded as the dominant microorganisms in refrigerated raw milk (Martins et al., 2006). Numerous genera of psychrotrophic bacteria have been isolated from raw milk consisting of both gram-negative bacteria (e.g. Pseudomonas, Achromobacter, Aeromonas, Serratia, Alcaligenes, Chromobacterium and Flavobacterium spp.) and gram-positive bacteria (e.g. Bacillus, Clostridum, Corynebacterium, Streptococcus, Lactobacillus and Microbacterium spp.). Between the two groups, gram-negative bacteria were isolated at a high frequency (Martins et al., 2006, Nornberg et al., 2010). Nornberg et al. (2010) reported that $90 \%$ of the psychrotrophic bacteria isolated from refrigerated raw milk from a processing plant in South Brazil was gram-negative based on morphological and biochemical tests. Using the random amplified polymorphic DNA (RAPD) fingerprinting technique, Martins et al. (2006) found that Pseudomonas spp. were predominant in raw milk in Brazil, too. In the U.S., Wiedmann et al. (2000) also reported Pseudomonas spp. were the predominant bacteria among the isolates from raw milk in New York State. Jayarao and Wang (1999) reported a significant presence of coliforms including Citrobacter spp., Enterobacter spp., Escherichia spp., and Klebsiella spp. in the bulk tank milk from South Dakota State.

Psychrotrophic bacteria are commonly predominant in refrigerated milk and produce heatresistant proteolytic and lipolytic enzymes, so they are the most important groups associated with milk quality (Marth and Steele, 2001). Microorganisms shown to produce heat-stable enzymes include Pseudomonas spp., Alcaligenes spp., Aerobacter spp., Achromobacter spp., and Serratia spp. (Griffiths et al., 1981). The degradation of protein by extracellular proteases can produce bitter-tasting peptides and cause curdling and clotting of milk; lactose can be fermented by psychrotrophic lactic acid bacteria resulting in sour flavor; the digestion of lipid by lipase and 
phospholipids creates short chain fatty acids that cause milk to be rancid (Sørhaug and Stepaniak, 1997).

In addition, spore-formers in raw milk should not be neglected. Spore-formers are widely present in food and cause different types of spoilage (Table 1). The spore-forming genera most relevant to milk and dairy products are Bacillus and Clostridium (Marth and Steele, 2001). Bacillus spp. account for $95 \%$ of the total spore-formers in milk while Clostridium species account for the remaining 5\% (Martin, 1974). Among all, B. licheniformis is the most predominant spore-former in raw milk; B. cereus is the most psychrotolerent spore-former despite seasonal, regional difference (Scheldeman et al., 2006). In addition, B. subtilis, B. pumilus, and B. amyloliqueciens etc. have been found in raw milk as well (De Jonghe et al., 2010). These spore-formers were capable of surviving High Temperature Short Time (HTST) processing, pasteurization and growth under refrigeration temperatures. Ultimately, they cause spoilage of processed products and shortened the shelf life (Huck et al., 2007). De Jonghe et al. (2010) investigated the effects on the quality of dairy products caused by spore-formers isolated from raw milk. Strains of Paenibacillus polymyxa, B. licheniformis, B. subtilis, B. amyloliquefaciens, B. pumilus, B. clausii and B. cereus are the main species of spore-formers that can impact milk quality. Among them, B. amyloliquefaciens, B. cereus, B. subtilis and P. polymyxa showed the greatest proteolytic effect; B. subtilis, B. pumilus and B. amyloliquefaciens showed significant lipolytic activity; $P$. polymyxa and B. cereus had lecithinase activity that hydrolyzes lecithin molecules present in the milk fat globule membrane, causing globule aggregation. Clostridium spp. have been implicated in the rancid spoilage and "late blowing" of numerous cheeses (Marth and Steele, 2001). 
Table 1. Spore-formers causing spoilage in foods (Scheldeman et al., 2006)

\begin{tabular}{lll}
\hline Species & Common food products affected & Product defect \\
\hline Alicyclobacillus acidoterrestris & Fruit juices, acidic beverages & Off-flavors \\
Bacillus cereus & Pasteurized milk, dairy products & $\begin{array}{l}\text { Bitty cream, } \\
\text { sweet curdling, } \\
\text { off-flavors }\end{array}$ \\
$\begin{array}{l}\text { Geobacillus stearothermophilus } \\
\text { Bacillus coagulans } \\
\text { Bacillus licheniformis }\end{array}$ & $\begin{array}{l}\text { Evaporated milk, low-acid } \\
\text { canned vegetable tomato juice }\end{array}$ & Flat sour \\
Bacillus subtilis & Bread & Ropy bread \\
\hline
\end{tabular}

Pathogens associating with mastitis are important microbial members in raw milk as well.

Several species have been described as mastitis-causing pathogens. These include

Staphylococcus aureus, Streptococcus uberis, Corynebacterium bovis, Escherichia coli,

Enterococcus and Arcanobacterium pyogenes (Park et al., 2007, Pitkala et al., 2004a, Taponen et

al., 2009). Mastitis is associated with microbial infection of a cow's udder. Milk components can

be dramatically changed because the pathogens use milk components as a substrate for growth.

For instance, in mastitic milk, fat content is reduced to below 3\%, chloride is increased by a

factor of 1.5, and lactose decreases substantially (Marth and Steele, 2001). Mastitis-causing

microorganisms can also have indirect effects on milk quality. Mastitis cows frequently shed $10^{6}$

somatic cells/ml to milk (Marth and Steele, 2001). Increased somatic cell count (SCC) is

correlated with increased amount of heat-stable protease (plasmin) and lipase (lipoprotein lipase)

in milk, which will cause protein and fat degradation resulting in off-flavors. Moreover, high

SCC can cause adverse effects on cheese quality including reducing curd firmness, decreasing 
cheese yield, increasing fat and casein loss in whey and compromising sensory quality (Barbano et al., 2006).

\subsubsection{Sources of bacterial contamination in raw milk}

For healthy cows, raw milk secreted into the alveoli of their udders is regarded free of microorganisms (Tolle, 1980). However, microorganisms are ubiquitous in the dairy environment and contaminate raw milk from three main sources: the dairy environment, within the udder and the milking equipment.

Dairy environmental sources, including soil, water, feed, feces and bedding material, vary in the numbers and types of microorganisms that can be transmitted into raw milk (Marth and Steele, 2001). The extent of contamination on the teat surface by environmental sources can determine the population of microorganisms in raw milk. For instance, milking heavily soiled cows could potentially result in bulk milk counts exceeding $10^{4} \mathrm{cfu} / \mathrm{ml}$ (Murphy and Boor, 2000). Various groups of microorganisms in raw milk have been found associated with the environmental sources. Contamination of coliform bacteria has been associated with manure and soil; sporeformers with bedding material and soil, and psychrotrophic microflora with soil, water, feeding and bedding material (Huck et al., 2008, Marth and Steele, 2001, Murphy and Boor, 2000).

Environmental sources contaminate raw milk through the exterior of the udder. Meanwhile, within the healthy udder, natural microflora of the healthy cow generally has little influence on the population of microorganisms. Raw milk harbors less than 1,000 total bacteria per ml as it leaves the udder of a healthy cow (Kurweil and Busse, 1973). However, a cow suffering mastitis caused by microorganisms can have high levels of microorganisms shed into raw milk. Cows 
infected with $S$. uberis can shed up to $10^{7} \mathrm{cfu} / \mathrm{ml}$ and cows infected with $E$. coli can shed up to $10^{8} \mathrm{cfu} / \mathrm{ml}$ (Marth and Steele, 2001).

Milking equipment is another common source of bacterial contamination for raw milk. Milk residue left on equipment contact surfaces provides a good environment for the growth of a variety of microorganisms. If cleaning procedures are ineffective, bacteria deposited in the equipment can multiply and becomes a major source of contamination (Marth and Steele, 2001).

\subsubsection{Factors influencing microbial community of raw milk}

Since the microorganisms originate from the three main sources, environment, mastitis and equipment, factors associated with them are able to affect microbial communities in raw milk potentially. Season is one of the main factors driving the microbial community structure. It has been reported that the predominant groups of microorganisms in raw milk varied with the season in Israel: Gammaproteobacteria were predominant in spring and winter, Bacilli in summer, and Actinobacteria in autumn (Hantsis-Zacharov and Halpern, 2007). In the U.K., according to Sutherland and Murdoch's study (1994), mesophilic spore counts in raw milk samples were higher in winter months (November to March) than in summer/autumn months (June to October) while psychrotrophic spore counts were conversely lower in the winter but higher in the late summer/autumn. In another study, Phillips and Griffiths (1986) also found that psychrotrophic spore-formers predominated in the summer-autumn months and resulted in the spoilage of pasteurized milk products (Phillips and Griffiths, 1986).

Seasonal fluctuation of microorganism populations in raw milk is expected. First, different genera have their own optimal temperature requirement. Bacillus species have relatively high optimal temperature growth while Gamaproteobacteria species have relatively lower optimal 
temperature growth. Hence, Bacillus were predominant in the summer and Gamaproteobacteria in winter and spring (Hantsis-Zacharov and Halpern, 2007). Second, the seasonal change brings about a change of weather patterns including humidity, temperature, wind, rain, etc. It can have direct impact on the predominant species in the dairy environment that will be transmitted to raw milk and cause the fluctuation of microbial communities in raw milk. For example, the increase in mesophilic spore counts from farm raw milk in winter corresponded to the increased mesophilic spores on udder surfaces due to contact with contaminated winter bedding (Sutherland and Murdoch, 1994). For San Luis Obispo County, precipitation is one of the weather patterns that changes substantially between seasons of autumn/winter (from November to March) and spring/summer (April to October) (Anonymous, 2010b). Thus in this study, we were interested in how the precipitation impacts the microbial community in raw milk. Meanwhile, weather is one of the uncontrollable factors in the dairy farm. With the understanding of its impact on the microbial community in raw milk, dairy farmers may take effective measures to safeguard raw milk quality.

Another factor capable of impacting the microbial community in raw milk is the cow's apparent health status. Mastitis, often caused by bacterial infection, is probably the most recognized disease that has a major impact on milk quality. Normally, although the microorganisms from healthy udder can be shed into the raw milk, the total bacteria count of raw milk is very low (Boor et al., 1998, Murphy and Boor, 2000) . In contrast, when cow's udders are infected by mastitis-causing pathogens, the population of microorganisms in raw milk can increase significantly (Marth and Steele, 2001). Many species of bacteria are reported as associated with mastitis (Table 2) and these species were different in different geographic areas. In the U.S., the clinical mastitis-causing organisms in New York State from 2003 to 2008 included gram-positive 
bacteria (Streptococcus spp., Staphylococcus spp., Staphylococcus spp.) and gram-negative bacteria (E. coli, Klebsiella spp., Citrobater spp.) (Hertl et al., 2010). In 2001, coagulasenegative staphylococci were still the most common bacterial group while the prevalence of Corynebacterium bovis increased compared to 1995 in Finland (Pitkala et al., 2004b). In Asia, Park et al. (2007) isolated coagulase-positive and negative staphylococci, Streptococcus spp., Enterococcus spp., E. coli and Pseudomonas spp. in raw milk in South Korea.

Table 2. Mastitis-causing microorganisms (Marth and Steele, 2001)

\section{Common agents:}

Staphylococcus aureus

Streptococcus spp. (especially S. agalacitiae, S. dysgalactiae, S. uberis)

Coliform bacteria (especially Escherichia coli, Citrobacter freundii, Enterobactor spp. and Klebsiella spp.)

Actinomycespyogenes

\section{Less common agents:}

Listeria monocytogenes

Pseudomonas aeruginosa

Mycoplasma bovis

Corynebacterium bovis and C. diphtheria

Nocardia spp. (especially N. asteroides)

Coagulase-negative Staphylococcus spp.

Bacillus cereus

Brucella abortus

Clostridium perfringens

Coxiella burnetii

Leptospira spp.

Mycobacterium bovis

Serratia marcesens

Prototheca zopfii (alga)

A cow's health status plays an important role in microbial community in raw milk and has the potential to impact the population of microorganisms in the milk. Consequently, health status 
may affect raw milk quality. Therefore, the health condition of cow's udders is another important factor that can affect raw milk quality and it was investigated in this study as well.

\subsection{Conventional tests}

\subsubsection{Introduction of conventional tests}

Since microorganisms in raw milk have the potential to impact the quality of dairy products including pasteurized milk and other final products, it is critical to evaluate the microbial quality of raw milk to ensure that only high quality raw milk is accepted so that final products will be of high quality. Various tests have been established for this purpose. In this study, these tests are referred to as "conventional tests" due to a long period of application in dairy industry. Nowadays, widely used conventional tests include standard plate count (SPC), coliform count (CC), lab pasteurized count (LPC), proteolytic strain count (PSC) and somatic cell count (SCC). They are desired to enumerate different microorganism groups such as total bacteria from SPC, coliform groups from CC, thermoduric bacteria from LPC and proteolytic bacteria from PSC (Pantoja et al., 2009).

SPC is the classic method to estimate the microbial population of most types of dairy products from raw milk to final products and to determine quality and source of microbial contamination at successive stages of processing (Wehr and Frank, 2004). It is commonly used to determine population of total bacteria, which is regarded as the most important parameter for raw milk quality evaluation. LPC is applied to estimate the population of thermoduric bacteria including Micrococcus, Microbacterium, Lactobacillus, Bacillus, Clostridium and Streptococcus.

Thermoduric bacteria can survive pasteurization, so they are usually associated with spoilage of 
pasteurized milk (Boor et al., 1998). CC is to estimate the population of coliform groups including several genera such as Escherichia, Enterobactor and Klebsiella, which are commonly associated with manure, soil and environmental contamination. $\mathrm{CC}$ is able to indicate both the effectiveness of cow preparation procedures during milking and cleanliness of the dairy environment because an important source of coliform bacteria in raw milk is the transferral of soil from teats and udders to the milking machine (Reinemann et al., 2003). Besides the bacterial counts, SCC is also used as an indicator of the milk quality given that the somatic cells release enzymes that are hydrolytic and may be heat-stable (Nielsen, 2002). As part of this study, a series of conventional tests consisting of SPC, CC, LPC, SCC and PSC were conducted for raw milk quality evaluation. The milk samples were classified into groups of high quality and low quality according to the results of conventional tests in our analyses.

The careful definition of thresholds is essential for a useful evaluation of milk quality. According to the PMO 2009 (Anonymous, 2009), in order to be qualified as "Grade A milk", total bacteria counts and somatic cell counts from individual farm must be $<100,000 \mathrm{cfu} / \mathrm{ml}$ and $<750,000$ cells/ml, respectively. There is no threshold for LPC and CC in PMO 2009. Raw milk designated as "Grade A" is produced under sufficiently sanitary conditions and is qualified for fluid consumption (Anonymous, 2009). These thresholds for maximum allowable bacteria and SCC are designed to ensure public health. Farmers are encouraged to reduce the bacterial count and SCC by milk payment system where the reduction of bacteria and somatic cells results in increased pay (Barbano et al., 2006). Thus, most milk in the U.S. in fact has much lower counts than the thresholds for Grade A milk. Research in North America has shown that uninfected cows have a mean SCC of approximately 70,000 cells/ml (Schukken et al., 2003); Boor et al. (1998) observed that the average SPC from 855 farms in New York state was approximately 
$11,000 \mathrm{cfu} / \mathrm{ml}$. Since it is possible to produce raw milk with relatively low levels of bacteria and somatic cells, more stringent criteria are set forth in some areas. For example, the maximum allowable total bacteria count and SCC for Grade A raw milk in California is specified to be $50,000 \mathrm{cfu} / \mathrm{ml}$ and 600,000 cells/ml, respectively (Anonymous, 2010a). In addition to SPC and SCC, LPC and CC are specified in California, both of which must be $<750 \mathrm{cfu} / \mathrm{ml}$ in Grade A raw milk. Nevertheless, in order to enhance raw milk quality, it is suggested that a value of 100 $\mathrm{cfu} / \mathrm{ml}$ for CC be used as an initial screening tool for raw milk (Marth and Steele, 2001). Regarding LPC, bulk tank milk with an LPC count $<200 \mathrm{cfu} / \mathrm{ml}$ is considered normal, while a count of $<10 \mathrm{cfu} / \mathrm{mL}$ indicates excellent equipment hygiene (Jayarao and Wolfgang, 2003). The usual LPC cutoff for raw milk quality is $200 \sim 300 \mathrm{cfu} / \mathrm{ml}$.

\subsubsection{Limitations of conventional microbial tests}

Conventional microbial tests have played a critical role in evaluating the quality of raw milk and milk products in past decades. However, those methods have some limitations.

First, most of the conventional tests are culture-dependent, which means that the viable but nonculturable (VBNC) microorganisms may not grow on the normally used culture media during the tests, thus they cannot be counted. The term VBNC is often used to describe cells that are metabolically active but cannot be cultured. The VBNC state can be a survival mechanism adopted by many bacteria when exposed to adverse environmental conditions. Alternatively, VBNC organisms may be injured bacteria that have lost the ability to grow on laboratory media (Gunasekera et al., 2003). However, these VBNC bacteria have the potential to cause quality defects of raw milk and milk products (Rowan, 2004). 
Second, there is no consensus on what combination of tests can produce results with the most relevance in assessing the quality of raw milk and milk products. For example, different combinations of tests are used in standards for Grade A raw milk. Standards of Grade A raw milk from the PMO 2009 are defined by the combination of SPC and SCC whereas the standards are defined by LPC and CC in addition to SPC and SCC in California (Anonymous, 2009, 2010a).

Third, conventional tests cannot provide sufficient information on the composition of microbial communities in raw milk. The bacterial counts can only detect the number of certain groups of microorganism such as coliform groups but they are not capable of revealing the general structure of microbial communities in raw milk at the level of genus/species.

Owing to these limitations, conventional tests are not able to provide sufficient reliability and efficiency for evaluating raw milk quality. It may be necessary to combine culture-dependent and -independent methods to generate a more accurate view of microbial ecology of raw milk (Delbes et al., 2007). To complement conventional methods, molecular methods were applied in this study.

\subsection{Terminal restriction fragment (TRF) analysis}

\subsubsection{Background}

Since microorganisms play a critical role in raw milk quality, it is essential to understand the microbial communities in milk samples in order to increase the accuracy and efficiency of milk quality evaluation. Aiming to obtain a comprehensive understanding of microbial communities, several PCR-based molecular typing methods have emerged (Hengstmann et al., 1999). These 
methods include Amplified Ribosomal DNA Restriction Analysis (ARDRA), Single Stranded Conformation Polymorphism analysis (SSCP), Thermal and Denaturing Gradient Gel Electrophoresis (TGGE and DGGE), Amplified Length Heterogeneity analysis (ALH) and Terminal Restriction Fragment (TRF) patterns (also known as T-RFLP analysis) (Kitts, 2001).

Denaturing Gradient Gel Electrophoresis (DGGE) of rDNA has become a useful tool for investigating microbial community in food after years of development (Ercolini, 2004). It is capable of separating the PCR amplicons of the same size but with different sequences. Separation is based on the decreased electrophoretic mobility of a partially melted doublestranded DNA molecule in polyacrylamide gels containing a linear gradient of DNA denaturants or a linear temperature gradient. DNA molecules with different sequences often have different melting temperatures and thus are immobilized at different positions in the gel (Muyzer and Smalla, 1998). With regards to dairy products, Coppola et al. (2001) used the PCR-DGGE with amplification of 16S V3 region to analyze the microbial diversity occurring in different types of Mozzarella Cheese in Italy. This study demonstrated that DGGE analysis is considered to be more discriminating than $16 \mathrm{~S}$ rDNA spacer polymorphism analysis and it is potentially suitable for analyzing the complex microbial community associated with dairy products (Coppola et al., 2001).

The SSCP method can also separate DNA fragments by sequence composition. After the DNA from samples is amplified by PCR, the amplicons are denatured into two single strand DNAs (ssDNA). The ssDNAs are subjected to nondenaturing polyacrylamide gel electrophoresis. The mobility of the ssDNA in the gel depends on its secondary structure which is determined by the nucleotide sequence (Widjojoatmodjo et al., 1994). The SSCP method has been employed to study dairy products. Duthoit et al. (2003) monitored the bacterial community dynamics of a raw 
milk cheese by the $16 \mathrm{~S}$ rRNA SSCP analysis and concluded that the SSCP was a powerful tool for monitoring the dynamics of global microbial population. Delbes et al. (2007) suggested that the SSPC could be used to produce complementary information to the culture-dependent approaches to milk microbial ecology (Delbes et al., 2007). With ARDRA, the total ribosomal DNA extracted from samples is amplified by PCR. Ideally, it yields a mixture of DNA fragments representing all species present in the samples. After PCR amplification, the amplicons are digested by restriction enzymes and analyzed by low resolution agarose gel electrophoresis (Smit et al., 1997). The TRF method is based on the principal of ARDRA with some modifications. As with ARDRA, TRF analysis entails DNA extraction, PCR amplification and restriction enzyme digestion. However, one of the primers used for TRF is labeled with a fluorescent dye so that the size of the terminal restriction fragment (TRF) can be detected and the amount can be quantified when the fragments are analyzed in an automated DNA sequencer equipped with a laser detector (Liu et al., 1997). TRF is now a common tool to study the microbial diversity of food samples. Rademaker et al. (2005) performed the TRF analysis to study the surface microflora composition and dynamics of bacterial smear-ripened Tilsit cheese. They demonstrated that TRF analysis had the specificity to follow the important members of the surface flora (Rademaker et al., 2005).

The PCR-based molecular technologies have been used to study the microbial community in food samples over the past two decades. They are capable of analyzing the microbial community with higher efficiency and accuracy compared to traditional culture-dependent method, but they have their own drawbacks. For the DGGE method, the major factor influencing its results was the inability to run a large number of samples on a single gel and the potential for variation between gels. As a consequence, the resolution was reduced and some information may be 
masked (Nunan et al., 2005). With regards to the SSCP method, its separation shown on the gels depends on the differences of secondary structure that are critically contingent on the conditions where the samples are analyzed, especially the temperature. Thus, the reproducibility of results may be questionable (Lockley and Bardsley, 2000). TRF has shown clear advantages over SSCP, DGGE and ARDRA (Marsh, 1999): First, the terminal fragment sizes generated from the digestion of amplicon pool can be compared with terminal fragments derived from a sequence database in order to obtain phylogenetic inference (Marsh, 1999). Second, TRF can produce results with higher resolution power than either SSCP or DGGE because the spreading gel used in TRF can differentiate fragments with a one bp size difference (Nunan et al., 2005). Third, TRF seems to be more suitable for routine analysis of large number of samples because it avoids gelto-gel variation, has high reproducibility and has been automated (Smalla et al., 2007). Considering the strengths of TRF method, it was chosen in this study.

\subsubsection{Methodology}

TRF analysis includes a series of procedures consisting of DNA extraction, PCR, restriction enzyme digestion and gel electrophoresis (Figure 1). Technologies associated with TRF have been improved in the past years since the method was developed in 1997 (Liu et al., 1997). 


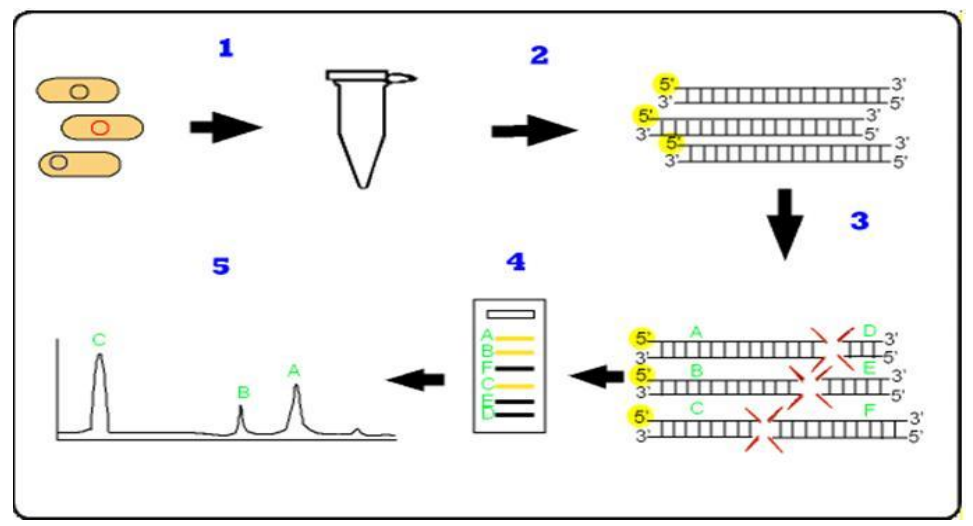

Figure 1. TRF analysis procedures (Gruntzig et al., 2002). 1. DNA extraction. 2. DNA amplification and fluorescent labeling. 3. Restrict enzyme digestion 4. Gel electrophoresis. 5. TRF profiles.

a. DNA extraction

DNA extraction is the initial step for the TRF method. Some milk components have negative effects on DNA extraction. Fat in raw milk foils efforts to obtain a consistent homogenate and extract adequate DNA from bacterial cells. It can be removed via centrifugation or organic extraction by diethyl ether (Ramesh et al., 2002). Casein micelles in raw milk can be digested by Proteinase $\mathrm{K}$ and release calcium ions that are able to result in severe DNA degradation due by activating DNase naturally present in raw milk. To improve the quality of genomic DNA, EDTA is commonly used prior to the utilization of Proteinase $\mathrm{K}$ to chelates calcium ions, dissolve casein micelles and eventually minimize the effect of DNase (Murphy et al., 2002). In order to obtain an accurate picture of the microbial diversity in a sample, DNA from all microbial species should be extracted effectively. An extraction protocol generally consists of three steps: cell lysis, removal of cell fragments and debris, and nucleic acid precipitation and purification. Among the microorganism in raw milk, gram-positive bacteria and spores are relatively resistant to lysis treatments. Therefore, cell lysis is a particularly critical step to ensure an effective DNA 
extraction. Methods to lyse cell wall and membrane include chemical, enzymatic and mechanical means (Table 3) (Roose-Amsaleg et al., 2001).

Table 3. Chemical, enzymatic and mechanical approaches for cell lysis

\begin{tabular}{ll}
\hline Categories & Common treatment \\
\hline Chemical & Detergent (SDS, Sarkosyl) \\
& Guanidiumisothiocyanate \\
& PVPP \\
CTAB \\
Sodium ascorbate \\
Enzymatic & Lysozyme \\
& Proteinase K \\
& Achromopeptidase \\
& Pronase \\
& Freeze-thaw cycles \\
Mechanical & Freeze-boil cycles \\
& Mortar mill grinding \\
& Bead-mill homogenization \\
& Bead-beating \\
& Microwave heating \\
& Sonication \\
\hline
\end{tabular}

Since spore-former microorganisms account for a considerable proportion of the microbial community in raw milk, spore DNA is important to the study of microbial community structure. Owing to their thick spore coat and layers of structure, spores are more resistant to lysis. Rueckert et al. (2005) modified procedures from Sambrook's protocol (Sambrook et al., 1989) and combined all the three lysis approaches to effectively release DNA from bacterial spores in milk power. In Rueckert's method, samples were treated by ultrasonic treatment followed by treatment with lysozyme, Proteinase K, 10\% SDS and then phenol/chloroform and chloroform/isoamyl alcohol extraction (Rueckert et al., 2005). Ultrasonic treatment has been proved to be an effective approach for spore lysis. Belgrader et al. (1999) found that the 
utilization of minisonicator could improve the PCR analysis of DNA from Bacillus spores by lowering the limit of detection, reducing the time and increasing the signal amplitude (Belgrader et al., 1999).

Commercial kits for DNA extraction are widely available nowadays, which are time-saving and less labor intensive. However, they may not work effectively on DNA extraction of raw milk samples since there is no commercial kit designed for milk samples particularly and their ability to extract spore DNA are questionable. Therefore, their effect on raw milk DNA extraction needs to be tested before applied.

With an improper DNA extraction method, the relative amount of DNA present in an extract can be biased by the presence of DNA unequally contributed by different types of microorganisms (Kitts, 2001). On the other side, overly vigorous cell lysis conditions needed to extract DNA from gram-positive bacteria and spores is not recommended because it may result in highly fragmented nucleic acids, subsequently causing artifacts during PCR (Wintzingerode et al., 1997). Hence, the optimization of the quality and quantity of DNA is a necessary and critical step to minimize the bias for the final results (Osborn et al., 2000).

\section{b. PCR}

After isolation and purification, DNA is subjected to PCR to amplify the target sequence, the conserved region of $16 \mathrm{~S}$ rDNA. In any PCR process, primers play an important role. Ideally, the primers chosen should be specific to the target taxonomic group yet sufficiently general to amplify all bacterial populations of interest (Schutte et al., 2008). However, there is no perfect primer satisfying the criteria. The analysis done using the Probe Match tool of the Ribosomal Database Project (RDP) showed that a single primer cannot amplify 60-99\% of the bacterial $16 \mathrm{~S}$ 
rRNA gene sequences and the analysis did not take into account that sequence databases only contain a fraction of the extant bacterial diversity. This suggests the "universal primers" commonly used now are far from universal (Marsh et al., 2000). Therefore, the option of primers is critical to enhance the accuracy of this method. Liu et al. (1997) found the primer pair $8 \mathrm{f}\left(5^{\prime}\right.$ AGAGTTTGATCCTGGCTCAG-3') and 926r (5'-CCGTCAATTCCTTTRAGTTT-3') followed by digestion with HhaI and MspI was able to classify the largest number of gene sequences into the largest number of unique 5' TRFs (Liu et al., 1997). Sakai et al. (2004) designed a new PCR primer set "783r". This primer set was an equimolar mixture of 783r-a (5'-

CTACCAGGGTATCTAATCCTG-3'), 783r-b (5' - CTACCGGGGTATCTAATCCC G-3'), and 783r-c (5'-CTACCCGGGTATCTAATCCGG-3') and able to eliminate the influence of DNA extraction in TRF analysis (Sakai et al., 2004).

Another important aspect is that one primer must be labeled fluorescently for laser detection. A commonly used tag in TRF analysis is 6-FAM (6-carboxyflourescein amino hexy fluorescent dye), but the specific tag depends on the manufacture's detection laser (Murillo, 2003). Since different bacterial population can share the same TRF pattern for a particular primer-enzyme combination, one labeled primer may result in underestimation of the microbial diversity in a sample. Given that, two or more labeled primers are currently used to enhance the resolution of TRF method (Schutte et al., 2008). Kitts (2001) suggested that an optimal amplicon length is between 400 to $700 \mathrm{bp}$ since this allows for the best possible estimation of microbial diversity while avoiding the loss of data associated with long amplicons (Kitts, 2001). 
c. Restriction enzyme digestion and gel electrophoresis

DNA amplicons are digested to produce a pattern of different length fragments after PCR.

Several points need to be taken into account when choosing the enzymes for digestion. Webbased tools are provided to select the appropriate enzymes for experiments such as T-RFLP analysis program (TAP) web site (http://rdp8.cme.msu.edu/html/TAP-trflp.html), MiCA (http://mica.ibest.uidaho.edu/) for 16S rRNA genes and the ARB implemented tool TRF-CUT for functional genes (http://www.mpi-marburg.mpg.de/downloads/) (Schutte et al., 2008). Since the organisms that produce identical length TRFs with one restriction enzyme may produce a different length when digested with another restriction enzyme, a more accurate community representation may be achieved by combing multiple TRFs from digestion with different enzymes (Clement et al., 1998). Osborne et al. (2006) concluded that the use of multiple restriction enzymes employed individually overcomes possible effects of restriction enzyme choice on generating useful TRF information for complex bacterial communities (Osborne et al., 2006). Therefore, two or more enzyme digestions are suggested to use simultaneously (Kitts, 2001).

Furthermore, the primer-enzyme combination can critically affect the number of TRF peaks. Liu et al. (1997) demonstrated that the primer pair 8f (5'-AGAGTTTGATCCTGGCTCAG-3') -926r (5' - CCGTCAATTCCTTTRAGTTT -3') followed by digestion with HhaI or MspI was able to obtain the largest number of TRF peaks (Liu et al., 1997). Nagashima et al. (2003) optimized this method and applied new primer-enzyme combination for two reasons: First, the primers were not suitable for amplification of 16S rDNA genes from some species of Bifidobacterium. Second, it is difficult to assign bacterial species identity to the TRF generated by $8 \mathrm{f}$ primer (Nagashima et al., 2003). In order to enhance the resolution, double enzyme digestion was tested. However, 
Dubar et al. (2001) demonstrated that even fewer phylogenetically informative TRFs were generated from double digestion than by combining results from separate single enzyme digests using enzyme MspI and RsaI. Thus the combination of single enzyme digests will appear to be the best strategy for the TRF approach (Dunbar et al., 2001). Capillary electrophoresis is widely used for the separation of digested DNA. The widely used automated DNA systems include the ABI Genetic Analyzer series, ABI Prism automated sequencer series, ALFexpress ${ }^{\mathrm{TM}}$ automatic sequence analyzer, and Beckman-Coulter CEQ series units (Danovaro et al., 2006, Lord et al., 2002, Rademaker et al., 2005, Wagner et al., 2009).

\subsubsection{Data analysis}

\section{a. Standardization of TRF profiles}

The first step to analyze TRF profile is to standardize the data and distinguish the signal from electronic noise. Either peak height or area has been used to distinguish signal from noise, and both have advantages and disadvantages (Schutte et al., 2008). Kitts (2001) suggested that peak area is the most accurate measure of DNA abundance in an electropherogram instead of peak height, because peak width increases as a function of retention time which means a standard amount of DNA fragments in different lengths will generate peaks with the same area but different heights.

A threshold is required to define which fluorescence data collected by the DNA sequencer will be retained for user analysis and which fluorescence data will be removed as noise. Sait et al. (2003) proposed a method using a constant percentage threshold. In this method, a matrix of all TRF peaks and their peak areas that are present in sample profiles from a given study is generated. If a TRF peak is not present in a particular profile, a value of zero is assigned. The 
dataset is divided by the total area across all peaks and the proportion is assigned to each peak in the profile. A percentage threshold is selected to determine the baseline in such a way so that the correlation between total peak area and number of peaks is minimized (Sait et al., 2003, Schutte et al., 2008). This standardization helps control the difference caused by the varying amount of injected DNA among different profiles. Since the exact amount of injected DNA is not known, this procedure is critical to ensure an objective analysis, especially for a comparison of different profiles (Kitts, 2001). Rees et al. (2004) suggest a 1\% threshold as a good starting point for analysis and an inappropriate threshold may cause the loss and/or bias of ecologically imperfect information (Rees et al., 2004).

\section{b. Peak Alignment of profiles}

The rounding method is the simplest and most common method for peak alignment. The estimated fragment size is rounded to the nearest integer and each integer is considered as a "bin" (Schutte et al., 2008). Dunbar et al. (2001) reported that this method gathers peaks within 0.5 bp and places only one peak from each sample into one bin (Dunbar et al., 2001). A potential drawback of this method is that identical fragments may be placed into different bins due to errors from electrophoresis despite visual inspection suggests that those peaks represent the same organism. To eliminate this drawback, manual binning could be a complement. It allows an experienced analyst to make intelligent choices in ambiguous cases. However, manual binning inevitably introduces subjective bias and the throughput is much lower than that of a computer (Schutte et al., 2008). To solve this problem, programs with more integration algorithms might be employed to analyze raw data. Computer-based programs are widely used since they can automate and facilitate binning procedures, for instance, $\mathrm{CEQ}^{\mathrm{TM}} 8000$ Genetic Analysis System uses the program "ALFP analysis" to perform this function. 


\section{c. Database matching}

Database matching can be used to tentatively identify specific populations in a microbial community. The identification can be conducted by comparing the length of sample TRFs to TRFs from a database of known bacteria that is created using the same primers and enzymes. Usually two or more enzyme digests are required for database matching to increase the resolution since one single TRF can represent more than one genus (Kitts, 2001).

The difficulty with using a database to identify a TRF peak is that the observed length does not always match with the predicted length. An approach to resolve this problem is to create a window, or a range, for matching. For example, a window of 5bp (from -1 bp to 4 bp) was utilized to identify Lactobacillus johnsonii in the rat fecal samples to compensate for the discrepancy between observed TRF lengths and those predicted by sequence analysis (Kaplan et al., 2001).

It is common that a large number of TRF peaks are generated from a set of samples, which leads to a large amount of computing for database matching. Therefore, web-based tools are a good choice for this work. Kent et al. (2003) developed a phylogenetic assignment tool (PAT) that rapidly generates phylogenetic assignments from submitted community TRF profiles based on a database of fragments produced by known $16 \mathrm{~S}$ rRNA gene sequences. Users are allowed to submit a customized database generated from unpublished sequences or from a gene other than the $16 \mathrm{~S}$ rRNAgene. This tool is helpful to employ TRF to analyze microbial community diversity and species composition rapidly because it can analyze the TRF profiles with multiple primer-enzyme combinations simultaneously (Kent et al., 2003). 


\subsubsection{Statistical tools utilized to analyze TRF data}

Statistical tools are indispensable to the microbial diversity analysis. To detect the dissimilarity among different microbial communities, a variety of the multivariate analyses have been used for data analysis. In this study, these statistical tools are used to analyze TRF data which represent the microbial communities in raw milk.

a. Non-parametric multivariate analysis of variance (NPMANOVA)

Statistical methods commonly applied in ecological studies were used to analyze our TRF data. In the standardized TRF data, abundance values are discrete rather than continuous. Moreover, abundance of small values is truncated at $1 \%$ during normalization and contributes a lot of zeros to the data set. Thus the parametric MANOVA methods are not applicable. NPMANOVA is a powerful and preferable tool in this regard. It is also used in most ecological multivariate data sets as their assumptions are less stringent than the parametric counterparts. NPMAOVA methods are based on measuring the distance or dissimilarity between pairs of individual multivariate observations or ranks. In addition, permutations of the observation are used to obtain a probability associated with the null hypothesis of no difference among groups.

Generally there are two categories of non-parametric methods. The first one is based on any chosen distance measure. For instance, the semimetric Bray-Curtis measure of ecological distance or Kulczyniki's semimetric measure are the most preferable methods (Anderson, 2001). But these methods are not capable of testing the variation across many factors. For complex designs, the data have to be analyzed as one-way analyses in multiple subsets within particular levels of factors. Given that, some non-parametric methods allowing partitioning for a complex 
design were applied, but the drawback of using these methods is that they are restricted to use with metric distance measures, which are not ideal for ecological applications.

Rees et al. (2004) introduced the Analysis of Similarity (ANOSIM) method to analyze TRF data to test the differences between microbial communities. ANOSIM was able to demonstrate the significance of differences and sensitive to differences in the dispersions of points. However, ANOSIM is not the most proper method for this study since it can analyze one factor only and more than one factor was involved in this study (e.g. rain event and cow's health status). The lack of generalized statistic for partitioning variation and appropriate permutation methods limits the application of ANOSIM here.

Anderson (2001) proposed an improved method for NPMANOVA to make up for the shortcoming of current methods. The assumption of this method is that the observations are exchangeable under a true null hypothesis, and this is generally satisfied in most ecological studies. This improved method can partition variation according to any ANOVA design; furthermore, it can be based on any symmetric dissimilarity or distance measure and provides a p-value using appropriate permutation methods.

In this study, multiple factors that may influence microbial community structure should be taken into account such as rain, cow's apparent health status. Anderson's method is currently the most suitable one to analyze the multifactor's effect on the microbial community and thus applied for TRF data analysis.

b. Similarity percentage (SIMPER)

In order to understand the dissimilarity between microbial communities, when raw milk samples were defined as two groups, SIMPER analysis was used to determine the contribution of each 
species to the average Bray-Curtis dissimilarity. In this study, SIMPER was used to analyze the dissimilarity between TRF data associated with raw milk samples in different groups.

The Bray-Curtis dissimilarity between any two groups of samples $j$ and $k$ was defined as below:

$$
\delta_{j k}=\sum_{i=1}^{p} \delta_{j k}(i)
$$

Where

$\delta_{\mathrm{jk}}(\mathrm{i})=100\left|\mathrm{y}_{\mathrm{ij}}-\mathrm{y}_{\mathrm{ik}}\right| / \sum_{\mathrm{i}=1}^{\mathrm{p}}\left(\mathrm{y}_{\mathrm{ij}}+\mathrm{y}_{\mathrm{ik}}\right)$,

$\mathrm{y}_{\mathrm{ij}}=$ The abundance of the $i$ th peak in the $j$ th sample,

$\mathrm{p}=$ The number of TRF peaks.

$\delta_{\mathrm{jk}}(\mathrm{i})$ is taken as the "contribution of the $i$ th peak" to $\delta_{\mathrm{jk}}$. Averaging $\delta_{\mathrm{jk}}$ over all sample pairs (j,k), with $j$ in the first group and $k$ in the second, gave the overall average dissimilarity, denoted as $\bar{\delta}$, between groups 1 and 2 . The same averaging taken over each $\delta_{\mathrm{jk}}(\mathrm{i})$ gave the average contribution $\bar{\delta}_{\mathrm{i}}$, from the $i$ th peak to the overall dissimilarity $\bar{\delta}$. Hence, a useful measure of the consistent contribution to $\bar{\delta}_{\mathrm{i}}$ from one peak was the standard deviation, $\operatorname{SD}\left(\bar{\delta}_{\mathrm{i}}\right)$ of the $\delta_{\mathrm{jk}}(\mathrm{i})$. If $\bar{\delta}_{\mathrm{i}}$ is large and $\operatorname{SD}\left(\bar{\delta}_{\mathrm{i}}\right)$ is small, then the $i$ th peak not only contributes much to the dissimilarity but also does so consistently, thus it is a good discriminating peak. For example, if some peaks could be found abundant in one group but largely absent from the other, these peaks may not be very useful discriminating ones due to their inconsistent contribution (Clarke, 1993). Herein, SIMPER was selected to analyze the dissimilarity between raw milk samples of different quality groups and to detect the contribution of each TRF peak to the dissimilarity. 


\subsubsection{Potential pitfalls of TRF analysis}

As a DNA fingerprinting method, the TRF method is a feasible option for analyzing the microbial diversity of samples. Besides, technology development improves the efficiency of this method. The application of automated capillary gel electrophoresis and web-based tools also facilitate the application of TRF analysis in a variety of research.

However, the potential pitfalls of TRF analysis should be taken into account when applied to raw milk quality analysis.

a. TRF analysis is not able to discriminate whether the bacteria are viable or not. DNA extracted from raw milk may originate from non-viable bacteria cells. Nevertheless, the nonviable bacteria consists of only a very small proportion of the total bacteria population in raw milk (Knaysi and Ford, 1938). Raw milk samples were collected in regular conditions that allow most of the bacteria to survive. Hence, although DNA from non-viable bacteria might be involved, TRF analysis is still acceptable and useful.

b. Artifacts and bias can be introduced into TRF patterns during DNA extration, PCR and electrophoresis. However, this holds true for almost all the microbial community analysis methods and therfore cannot be considered a disadvantage specific to TRF analysis (Kitts, 2001).

c. DNA used for TRF analysis cannot be reclaimed. To overcome this drawback, more sample amount should be collected to make sure sufficient or excess DNA is extracted for all the tests. For our industry, fortunately, raw milk is generally adequate and inexpensive. 
In conclusion, owing to the relatively high resolution and high efficiency among the PCR-based molecular typing methods, TRF analysis was employed to investigate the microbial community structure in our raw milk samples and to complement conventional tests in evaluating raw milk quality. 


\section{Chapter 3 Methods and materials}

\subsection{Milk sample collection}

Thirty-six (36) raw milk samples (Table 4) were collected from cows in the dairy unit of the California Polytechnic State University, San Luis Obispo. Containers and milking equipment were sterilized before sampling. One liter of each raw milk sample was obtained from individual cows from 3:00 pm to 5:00 pm and was transported immediately to Dairy Products Technology Center (DPTC) in an ice-packed cooler. In each sampling, samples were collected from 6 to 8 cows. Samples in December and January were collected on wet days (rainy days) while others were collected on dry days (sunny days). Meanwhile, approximately half of the cows sampled were apparent healthy and the others were apparent unhealthy. "Unhealthy cows" referred to those manifested disease symptoms including mastitis and other apparent udder inflammation observed by dairy farmers. After collection, all samples were divided in half: one portion was used for the conventional tests within $24 \mathrm{~h}$ and the other frozen was at $-20{ }^{\circ} \mathrm{C}$ for later TRF analysis.

Table 4. Raw milk samples information

\begin{tabular}{ccccc}
\hline Sample No. & Cow No. & $\begin{array}{c}\text { Date of sample } \\
\text { collection }\end{array}$ & Rain event & Apparent health condition \\
\hline $12-154$ & 154 & December, 2009 & Wet & Healthy \\
$12-157$ & 157 & December, 2009 & Wet & Healthy \\
$12-158$ & 158 & December, 2009 & Wet & Healthy \\
$12-213$ & 213 & December, 2009 & Wet & Unhealthy \\
$12-278$ & 278 & December, 2009 & Wet & Unhealthy \\
$12-703$ & 703 & December, 2009 & Wet & Unhealthy \\
$1-167$ & 167 & January, 2010 & Wet & Unhealthy \\
$1-201$ & 201 & January, 2010 & Wet & Unhealthy \\
\hline
\end{tabular}


Continued Table 4

\begin{tabular}{lllll}
\hline $1-240$ & 240 & January, 2010 & Wet & Healthy \\
$1-243$ & 243 & January, 2010 & Wet & Healthy \\
$1-268$ & 268 & January, 2010 & Wet & Healthy \\
$1-807$ & 807 & January, 2010 & Wet & Unhealthy \\
$1-844$ & 844 & January, 2010 & Wet & Unhealthy \\
$3-183$ & 183 & March, 2010 & Dry & Healthy \\
$3-193$ & 193 & March, 2010 & Dry & Healthy \\
$3-201$ & 201 & March, 2010 & Dry & Unhealthy \\
$3-225$ & 225 & March, 2010 & Dry & Unhealthy \\
$3-240$ & 240 & March, 2010 & Dry & Unhealthy \\
$3-243$ & 243 & March, 2010 & Dry & Healthy \\
$3-807$ & 807 & March, 2010 & Dry & Unhealthy \\
$3-844$ & 844 & March, 2010 & Dry & Unhealthy \\
$5-111$ & 111 & May, 2010 & Dry & Unhealthy \\
$5-179$ & 179 & May, 2010 & Dry & Unhealthy \\
$5-190$ & 190 & May, 2010 & Dry & Healthy \\
$5-205$ & 205 & May, 2010 & Dry & Healthy \\
$5-209$ & 209 & May, 2010 & Dry & Healthy \\
$5-259$ & 259 & May, 2010 & Dry & Unhealthy \\
$5-703$ & 703 & May, 2010 & Dry & Unhealthy \\
$7-111$ & 111 & July, 2010 & Dry & Unhealthy \\
$7-179$ & 179 & July, 2010 & Dry & Unhealthy \\
$7-190$ & 190 & July, 2010 & Dry & Healthy \\
$7-205$ & 205 & July, 2010 & Dry & Healthy \\
$7-209$ & 209 & July, 2010 & Dry & Healthy \\
$7-212$ & 212 & July, 2010 & Dry & Healthy \\
$7-255$ & 255 & July, 2010 & Dry & Unhealthy \\
$7-844$ & 844 & July, 2010 & Dry & Unhealthy \\
\hline & & & &
\end{tabular}

\subsection{Conventional tests}

SPC was conducted following "Standard methods for the examination of dairy products" (Wehr and Frank, 2004). All milk samples were diluted in phosphate buffer. One hundred microliters of each diluted sample was plated on Difco ${ }^{\mathrm{TM}}$ standard method agar (BD, Franklin Lakes, NJ) and spread evenly with a sterile rod. All plating was made in duplicate. Plates were incubated at $32{ }^{\circ} \mathrm{C}$ for $48 \mathrm{~h}$. 
All plates with $25-250$ colonies were counted and $\mathrm{cfu} / \mathrm{ml}$ was enumerated using the following equation: $\mathrm{N}=\mathrm{C} /([(1 \times \mathrm{n} 1)+(0.1 \times \mathrm{n} 2)] \times \mathrm{d})$

Where:

$\mathrm{N}=\mathrm{cfu} / \mathrm{ml}$

$\mathrm{C}=$ number of colonies from both plates with 25-250 colonies

$\mathrm{n} 1=$ number of plates in lower dilution counted

$\mathrm{n} 2=$ number of plates in next higher dilution counted

$\mathrm{d}=$ dilution from which the first counts were obtained.

For LPC, thirty milliliter of each raw milk sample was heated up to $63^{\circ} \mathrm{C}$ in water bath and held for $30 \mathrm{~min}$ at the same temperature, followed by the Standard Plate Count procedures. CC was conducted by using $3 \mathrm{M}^{\mathrm{TM}}$ Petrifilm ${ }^{\mathrm{TM}}$ Coliform (3M, St. Paul, MN) following the manufacture's instruction. Petrifilms were incubated at $32{ }^{\circ} \mathrm{C}$ for $24 \mathrm{~h}$ and colonies were counted. SCC was conducted by using Delaval somatic cell counter (Delaval, Kansas City, MO). The results were reported as cells/ $\mu 1$, which was converted to cells/ml. For PSC, standard method caseinate ager was prepared using standard method agar as a base. One liter medium contained $23.5 \mathrm{~g}$ Standard Method Agar and $4.41 \mathrm{~g}$ trisodium citrate. Before plating, $20 \mathrm{ml}$ of sterile $1 \mathrm{M}$ calcium chloride was added. Standard plate counting procedures were followed with incubation of $72 \mathrm{~h}$. Colonies surrounded by a white or off-white zone of paracasein precipitation are proteolytic. Highly proteloytic bacteria also produced a clear inner zone. Besides, $\mathrm{pH}$ of each sample was tested at room temperature $\mathrm{pH}$ meter was calibrated before tests. All of the conventional tests were conducted within $24 \mathrm{~h}$ after sampling. 


\subsection{Milk quality evaluation according to conventional tests}

Raw milk quality was evaluated according to each conventional test respectively. A set of cutoffs was applied as shown in Table 5. In our study, the results of conventional tests were mostly lower than the criteria of Grade A raw milk, thus lower cutoffs were used according to the section 2.2.1 in order to avoid a highly skewed distribution of the number of high and low quality milk samples. Milk quality was evaluated by each test respectively. Raw milk samples with bacterial/somatic cell count less than the cutoff were defined as "high quality milk" while the ones with bacterial/somatic cell count greater than the cutoff were defined as "low quality milk".

Table 5. Cutoffs of conventional tests for milk quality evaluation

\begin{tabular}{|l|l|l|l|l|}
\hline Conventional tests & SPC & SCC & CC & LPC \\
\hline Cutoff & $50,000 \mathrm{cfu} / \mathrm{ml}$ & $70,000 \mathrm{cell} / \mathrm{s} / \mathrm{ml}$ & $100 \mathrm{cfu} / \mathrm{ml}$ & $250 \mathrm{cfu} / \mathrm{ml}$ \\
\hline
\end{tabular}

\subsection{TRF analysis}

\subsubsection{Optimization of DNA extraction methods}

Raw milk samples were autoclaved at $121{ }^{\circ} \mathrm{C}$ for 15 min to prevent microorganisms originally present in raw milk from proliferating and contributing more DNA. Spores of Bacillus stearothermophilus (ATCC 12980), Bacillus licheniformis (ATCC 14680) and Bacillus megaterium (ATCC 14581) were added in autoclaved milk in equal amount to reach the concentration of $10^{6}$ spores $/ \mathrm{ml}$ milk. Ten milliliters milk samples containing $10^{5}, 10^{4}, 10^{3}$ or $10^{2}$ spores were centrifuged at $4000 \mathrm{RPM}$ at $4^{\circ} \mathrm{C}$ for $20 \mathrm{~min}$. The pellet was resuspended in $0.5 \mathrm{ml}$ of $50 \mathrm{mM}$ TrisHCl (pH8.0), $100 \mathrm{mM} \mathrm{NaCl}$ and $100 \mathrm{mM}$ EDTA. 
Following the pretreatment, the traditional DNA extraction method and a commercial DNA extraction kit were applied for optimization as below.

\section{a. Method 1}

Suspension from last step was used for DNA extraction by MoBio PowerSoil DNA isolation Kit (MoBio Laboratories, Inc. CA) following the manufacture's instruction. DNA was quantified and stored at $-20^{\circ} \mathrm{C}$ subsequently for further use.

\section{b. Method 2}

The suspension was sonicated at level 5 for 20 shoots by the sonicator. Lysozyme (3.75mg) was added and samples incubated at $37^{\circ} \mathrm{C}$ for $30 \mathrm{~min}$. SDS and proteinase $\mathrm{K}$ were added at $1 \%(\mathrm{w} / \mathrm{v})$ and $200 \mu \mathrm{g} / \mathrm{ml}$ respectively. After incubation at $55^{\circ} \mathrm{C}$ for $1 \mathrm{~h}$, samples were treated for the first time with equal volumes of phenol/chloroform (1:1) followed by chloroform/isoamyl (24:1). DNA was then precipitated by addition of $1: 10(\mathrm{v} / \mathrm{v})$ of $3 \mathrm{M}$ sodium acetate and $0.6(\mathrm{v} / \mathrm{v})$ of isopropanol and incubated at $-20{ }^{\circ} \mathrm{C}$ for $20 \mathrm{~min}$. After $20 \mathrm{~min}$ centrifugation at $4{ }^{\circ} \mathrm{C}$, the DNA pellets were washed twice with chilled $80 \%$ ethanol, air-dried and resupended in $200 \mu 1$ of $1 \times \mathrm{TE}$ (10 mM Tris-HCl, $1 \mathrm{mM}$ EDTA, pH 8.0). Two microliters RNase was added and DNA samples were incubated at $37^{\circ} \mathrm{C}$ for $30 \mathrm{~min}$. Thereafter, DNA was purified and recovered using phenol/chloroform, chloroform and chloroform/isoamyl alcohol extraction and precipitation. Finally, DNA pellets were resuspended in $100 \mu 11 \times \mathrm{TE}$ for quantification and subsequently stored at $-20{ }^{\circ} \mathrm{C}$ for further use. 
For both methods, the Invitrogen Qubit ${ }^{\mathrm{TM}}$ Quantitation Platform (Invitrogen, Carlsbad, CA) was used to quantify DNA following manufacturer's instruction. DNA concentration was reported as $\mathrm{ng} / \mu \mathrm{l}$.

Method 2 was chose for all further experiments due to a higher yield of DNA extracted that Method 1 (results shown in Section 4.1). Genomic DNA was extracted from $200 \mathrm{ml}$ of each milk sample using Method 2, quantified and then stored frozen at $-20{ }^{\circ} \mathrm{C}$ until PCR processing.

\subsubsection{S rDNA PCR amplication}

The products of three PCR replicates were combined and each contained: 10 ng DNA, $1 \mu$ of 10 $\mu \mathrm{M}$ forward and $1 \mu \mathrm{l}$ of $10 \mu \mathrm{M}$ reverse universal primers, 2 Illustra PuReTaq Ready-To-Go ${ }^{\mathrm{TM}}$ RT-PCR Beads (GE health care, Piscataway, NJ) together released 2.5 units of PuReTaq DNA polymerase, $10 \mathrm{mM}$ Tris- $\mathrm{HCl}, 50 \mathrm{mM} \mathrm{KCl}, 1.5 \mathrm{mM} \mathrm{MgCl} 2,200 \mu \mathrm{M}$ of each dNTP, stabilizers, and BSA in the final volume of $50 \mu \mathrm{l}$ brought by PCR-grade water. The reverse primer was K2R (5'-GTATTACCGCTGCTG G-3') and forward primer was 8dF (5'-

AGAGTTTGTTCMTGGCTCAG-3'). The forward primer was labeled with a Cy5 fluorescent tag for the detection in capillary gel electrophoresis. Thermocycling was performed on a PCR system following the steps below:

$94^{\circ} \mathrm{C}$ for $10 \mathrm{~min}, 30$ cycles of $94^{\circ} \mathrm{C}$ for $1 \mathrm{~min}, 46.5^{\circ} \mathrm{C}$ for $1 \mathrm{~min}$, and $72^{\circ} \mathrm{C}$ for $2 \mathrm{~min}$, and finally a 10 min extension at $72^{\circ} \mathrm{C}$.

All products were cleaned with the MoBio ${ }^{\circledR}$ Ultra Clean PCR Clean-Up Kit and quantified using the BioTek Fluorometer (BioTek, Winooski, VT). 


\subsubsection{Enzyme digestion}

One hundred nanograms aliquot of $16 \mathrm{~S}$ rDNA amplicon was digested with restriction enzymes (New England Biolabs, Beverly, MA). For DpnII, $1 \mu$ l enzyme and $4 \mu$ l buffer was used per reaction while $0.5 \mu 1$ enzyme and $4 \mu \mathrm{l}$ buffer was used for HaeIII. PCR-grade water was added to bring the volume to $40 \mu \mathrm{l}$. Reactions were maintained at $37^{\circ} \mathrm{C}$ for $4 \mathrm{~h}$ then $80^{\circ} \mathrm{C}$ for $20 \mathrm{~min}$ to deactivate the enzyme. Samples collected in December 2009 and January 2010 were digested by DpnII only while the other samples were digested by both DpnII and HaeIII. Digests were stored in a $-20^{\circ} \mathrm{C}$ freezer until ready for ethanol precipitation.

\subsubsection{Excess salts removal}

One hundred microliters $(100 \mu \mathrm{l})$ of cold $95 \%$ ethanol, $2 \mu \mathrm{l}$ of $3 \mathrm{M}$ sodium acetate and $1 \mu \mathrm{g}$ lycogen $(20 \mathrm{mg} / \mathrm{ml})$ was added to each digest. Samples were placed in the $-20^{\circ} \mathrm{C}$ freezer for 30 min and then centrifuged at 5300 RPM for $15 \mathrm{~min}$. Ethanol was removed and $100 \mu$ cold $70 \%$ cold ethanol was added per digest. Samples were centrifuged again at 5300 RPM for 5 min and decanted. Then the DNA pellet was dried by centrifugation at 700 RPM for 1 min. DNA samples were stored in the $-20^{\circ} \mathrm{C}$ until the next step.

\subsubsection{Labeled digested fragments.}

Master mix ( $20 \mu \mathrm{l}$ formamide and $0.25 \mu \mathrm{l} 600$ base pair standard) was added to each reaction. One drop of mineral oil was added to the top of each well to prevent sample evaporation. Then samples were run on the $\mathrm{CEQ}^{\mathrm{TM}} 8000$ Genetic Analysis System (Beckman Coulter Inc, Fullerton, CA). Data were generated from the program “AFLP ${ }^{\circledR}$ analysis" following the manufacture’s instruction (Beckman Coulter Inc, Fullerton, CA). 


\subsection{Statistical analysis}

For the conventional tests, correlation analyses between the different microbiological parameters were performed on log-transformed data using Microsoft Excel (2007). Variables including SPC, CC, LPC, PSC, SCC and pH were subject to analyses. PCA and MDS were used to visualize the difference between samples grouped by rain and cow's apparent health. MANOVA was applied to detect significance of the difference between groups. In order to satisfy the assumption of normality for MANOVA, the transformation of logarithm with the base of 10 was conducted to the SPC, SCC, CC, LPC and PSC data (Appendix 3). Following MANOVA, ANOVA was conducted to detect the influence of each variable.

For TRF analysis, TRF peak alignment was conducted by the software "AFLP ${ }^{\circledR}$ analysis" which permitted binning of TRF peaks with respect to base pair size. Since the total area of TRF peaks varied among samples, the total area of all TRF peaks from each sample was normalized to parts per million before analysis. All the peaks comprising less than $1 \%$ of total area $(10,000 \mathrm{ppm})$ were abandoned. Next, the TRF data generated by $D p n$ II was exported to statistics software for further analysis. NPMANOVA by Anderson (2001) was applied to detect significant differences in TRF data between milk samples grouped by quality (as defined by conventional tests) and by the factors of rain event and cow's apparent health. SIMPER analysis was used to investigate each TRF peak's contribution to the total dissimilarity.

Database matching was conducted with TRF data from samples digested by both DpnII and HaeIII. The results were used as references for the samples digested by DpnII only. These TRF data were assigned to the closest matches of corresponding genus or species according to Genbank (Benson et al., 1998) using the TRF phylogenetic assignment tool (PAT: 
http://trflp.limnology.wisc.edu). TRF peaks of less than 400 base pairs were assigned to the database-recorded length within \pm 1.0 base pair. For longer TRFs ( $>400$ base pairs), the assignment was made to \pm 2.0 base pairs (Tanaka et al., 2010).

PCA and ANOVA were conducted by using Minitab 16 (McKenzie, 1999); MDS, SIMER by using PRIMER V5 (PRIMER-E ltd, UK), and MANOVA, NPMANOVA by using R (R Development CoreR et al., 2010). 


\section{Chapter 4 RESULTS}

\subsection{Optimization of DNA extraction}

The DNA yield of Method 1 and Method 2 were compared. The detection limit was $1.0 \times 10^{4}$ spores $/ \mathrm{ml}$ for Method 1 and $1.0 \times 10^{2}$ spores $/ \mathrm{ml}$ for Method 2. Even at the level of $1.0 \times 10^{4}$ spores/ml, Method 2 showed a much higher DNA yield $(0.729 \mathrm{ng} / \mu \mathrm{l})$ than Method $1(0.0234$ $\mathrm{ng} / \mu \mathrm{l})$. Method 2 was more sensitive and effective on spore DNA extraction. Therefore, it was applied in this study.

\subsection{Results of conventional tests}

The results of conventional tests were shown in Table 6. Correlation coefficients between pairs for different conventional tests were calculated (Table 7). The correlation between SPC and CC, and SPC and PSC were found higher than 0.8. The highest correlation was between SPC and PSC. The correlation between SCC and other tests were negative or positive depending on the tests, yet the absolute values were only approximately 0.1 . 
Table 6. Results of conventional tests

\begin{tabular}{|c|c|c|c|c|c|c|}
\hline Cow No. & $\mathrm{pH}$ & $\mathrm{SPC}(\mathrm{cfu} / \mathrm{ml}$ & $\mathrm{CC}($ cells $/ \mathrm{ml})$ & $\mathrm{CC}(\mathrm{cfu} / \mathrm{ml})$ & LPC (cfu/ml) & PSC (cfu/ml) \\
\hline $12-154$ & 6.78 & $2.5 \mathrm{E}+05$ & $8.4 \mathrm{E}+04$ & $3.2 \mathrm{E}+03$ & $5.8 \mathrm{E}+02$ & $1.0 \mathrm{E}+05$ \\
\hline $12-157$ & 6.7 & $4.9 \mathrm{E}+05$ & $5.5 \mathrm{E}+04$ & $1.1 \mathrm{E}+04$ & $5.4 \mathrm{E}+02$ & $1.4 \mathrm{E}+05$ \\
\hline $12-158$ & 6.85 & $5.5 \mathrm{E}+05$ & $8.8 \mathrm{E}+04$ & $1.7 \mathrm{E}+04$ & $3.0 \mathrm{E}+03$ & $1.1 \mathrm{E}+05$ \\
\hline $12-213$ & 6.73 & $1.0 \mathrm{E}+06$ & $1.2 \mathrm{E}+04$ & $6.8 \mathrm{E}+04$ & $2.6 \mathrm{E}+02$ & $1.7 \mathrm{E}+05$ \\
\hline $12-278$ & 6.75 & $2.4 \mathrm{E}+05$ & $6.5 \mathrm{E}+04$ & $1.3 \mathrm{E}+03$ & $3.0 \mathrm{E}+02$ & $8.5 \mathrm{E}+04$ \\
\hline $12-703$ & 6.76 & $2.2 \mathrm{E}+05$ & $2.3 \mathrm{E}+06$ & $3.6 \mathrm{E}+03$ & $3.6 \mathrm{E}+02$ & $7.5 \mathrm{E}+04$ \\
\hline $1-167$ & 6.7 & $2.3 \mathrm{E}+05$ & $9.0 \mathrm{E}+03$ & $1.2 \mathrm{E}+03$ & $6.5 \mathrm{E}+01$ & $3.1 \mathrm{E}+04$ \\
\hline $1-201$ & 6.64 & $1.3 \mathrm{E}+05$ & $1.8 \mathrm{E}+04$ & $1.7 \mathrm{E}+03$ & $6.8 \mathrm{E}+01$ & $2.0 \mathrm{E}+04$ \\
\hline $1-240$ & 6.69 & $2.0 \mathrm{E}+05$ & $1.5 \mathrm{E}+04$ & $9.5 \mathrm{E}+02$ & $5.7 \mathrm{E}+01$ & $1.6 \mathrm{E}+04$ \\
\hline $1-243$ & 6.71 & $1.3 \mathrm{E}+04$ & $7.5 \mathrm{E}+03$ & $3.2 \mathrm{E}+02$ & $5.3 \mathrm{E}+01$ & $7.5 \mathrm{E}+03$ \\
\hline $1-268$ & 6.73 & $1.2 \mathrm{E}+04$ & $1.7 \mathrm{E}+04$ & $3.0 \mathrm{E}+01$ & $1.6 \mathrm{E}+01$ & $7.5 \mathrm{E}+03$ \\
\hline $1-807$ & 6.76 & $7.4 \mathrm{E}+04$ & $1.0 \mathrm{E}+06$ & $8.0 \mathrm{E}+03$ & $2.0 \mathrm{E}+01$ & $1.8 \mathrm{E}+04$ \\
\hline $1-844$ & 6.81 & $1.0 \mathrm{E}+05$ & $4.1 \mathrm{E}+05$ & $1.2 \mathrm{E}+03$ & $2.5 \mathrm{E}+01$ & $3.8 \mathrm{E}+04$ \\
\hline $3-183$ & 6.92 & $1.6 \mathrm{E}+04$ & $8.3 \mathrm{E}+04$ & $1.0 \mathrm{E}+01$ & $7.4 \mathrm{E}+02$ & $2.1 \mathrm{E}+03$ \\
\hline $3-193$ & 7.12 & $1.1 \mathrm{E}+04$ & $2.9 \mathrm{E}+05$ & $2.3 \mathrm{E}+01$ & $3.8 \mathrm{E}+02$ & $2.0 \mathrm{E}+03$ \\
\hline 3-201 & 6.79 & $1.1 \mathrm{E}+04$ & $1.8 \mathrm{E}+04$ & $5.0 \mathrm{E}+00$ & $6.3 \mathrm{E}+02$ & $1.8 \mathrm{E}+03$ \\
\hline $3-225$ & 6.93 & $1.1 \mathrm{E}+04$ & $2.5 \mathrm{E}+04$ & $6.6 \mathrm{E}+02$ & $6.1 \mathrm{E}+02$ & $2.1 \mathrm{E}+03$ \\
\hline $3-240$ & 6.91 & $5.0 \mathrm{E}+04$ & $7.7 \mathrm{E}+03$ & $1.5 \mathrm{E}+03$ & $7.5 \mathrm{E}+02$ & $5.5 \mathrm{E}+03$ \\
\hline $3-243$ & 6.82 & $1.2 \mathrm{E}+04$ & $8.3 \mathrm{E}+03$ & $1.4 \mathrm{E}+01$ & $5.0 \mathrm{E}+02$ & $1.8 \mathrm{E}+03$ \\
\hline $3-807$ & 6.88 & $9.2 \mathrm{E}+03$ & $1.3 \mathrm{E}+05$ & $1.0 \mathrm{E}+01$ & $5.2 \mathrm{E}+02$ & $5.0 \mathrm{E}+03$ \\
\hline $3-844$ & 6.98 & $1.1 \mathrm{E}+05$ & $1.3 \mathrm{E}+05$ & $3.0 \mathrm{E}+01$ & $4.2 \mathrm{E}+03$ & $2.2 \mathrm{E}+04$ \\
\hline $5-111$ & 7.11 & $1.2 \mathrm{E}+04$ & $1.1 \mathrm{E}+06$ & $1.4 \mathrm{E}+01$ & $3.0 \mathrm{E}+02$ & $8.0 \mathrm{E}+03$ \\
\hline $5-179$ & 6.77 & $1.9 \mathrm{E}+03$ & $1.8 \mathrm{E}+05$ & $2.7 \mathrm{E}+01$ & $9.0 \mathrm{E}+01$ & $1.9 \mathrm{E}+03$ \\
\hline $5-190$ & 6.78 & $5.3 \mathrm{E}+03$ & $3.8 \mathrm{E}+04$ & $3.0 \mathrm{E}+00$ & $1.6 \mathrm{E}+02$ & $3.0 \mathrm{E}+03$ \\
\hline $5-205$ & 6.73 & $7.8 \mathrm{E}+03$ & $9.0 \mathrm{E}+03$ & $3.1 \mathrm{E}+01$ & $5.0 \mathrm{E}+01$ & $4.0 \mathrm{E}+04$ \\
\hline $5-209$ & 6.83 & $3.6 \mathrm{E}+03$ & $1.6 \mathrm{E}+04$ & $8.0 \mathrm{E}+00$ & $8.0 \mathrm{E}+01$ & $3.3 \mathrm{E}+03$ \\
\hline $5-259$ & 6.81 & $7.2 \mathrm{E}+03$ & $4.8 \mathrm{E}+05$ & $1.3 \mathrm{E}+02$ & $1.3 \mathrm{E}+02$ & $1.8 \mathrm{E}+03$ \\
\hline $5-703$ & 6.91 & $1.4 \mathrm{E}+04$ & $4.0 \mathrm{E}+06$ & $5.8 \mathrm{E}+02$ & $1.1 \mathrm{E}+02$ & $3.0 \mathrm{E}+03$ \\
\hline $7-111$ & 7.25 & $1.8 \mathrm{E}+04$ & $6.9 \mathrm{E}+05$ & $2.0 \mathrm{E}+00$ & $7.9 \mathrm{E}+02$ & $1.6 \mathrm{E}+04$ \\
\hline $7-179$ & 6.69 & $3.0 \mathrm{E}+04$ & $6.3 \mathrm{E}+04$ & $1.3 \mathrm{E}+02$ & $3.3 \mathrm{E}+02$ & $5.9 \mathrm{E}+03$ \\
\hline $7-190$ & 6.72 & $2.5 \mathrm{E}+03$ & $6.7 \mathrm{E}+04$ & $1.8 \mathrm{E}+01$ & $1.9 \mathrm{E}+02$ & $1.0 \mathrm{E}+04$ \\
\hline $7-205$ & 6.66 & $6.5 \mathrm{E}+03$ & $8.5 \mathrm{E}+03$ & $7.0 \mathrm{E}+00$ & $1.2 \mathrm{E}+02$ & $1.1 \mathrm{E}+03$ \\
\hline $7-209$ & 6.71 & $1.3 \mathrm{E}+03$ & $4.5 \mathrm{E}+04$ & $3.0 \mathrm{E}+00$ & $9.0 \mathrm{E}+01$ & $4.0 \mathrm{E}+02$ \\
\hline $7-212$ & 6.7 & $3.6 \mathrm{E}+03$ & $7.6 \mathrm{E}+04$ & $3.0 \mathrm{E}+00$ & $1.9 \mathrm{E}+02$ & $4.5 \mathrm{E}+02$ \\
\hline $7-255$ & 7.03 & $6.8 \mathrm{E}+03$ & $1.3 \mathrm{E}+06$ & $4.0 \mathrm{E}+01$ & $2.8 \mathrm{E}+02$ & $2.7 \mathrm{E}+03$ \\
\hline $7-844$ & 6.83 & $1.3 \mathrm{E}+04$ & $7.6 \mathrm{E}+05$ & $4.0 \mathrm{E}+00$ & $2.1 \mathrm{E}+02$ & $5.9 \mathrm{E}+03$ \\
\hline
\end{tabular}


Table 7. Correlation coefficients between results of different conventional tests.

\begin{tabular}{llllll}
\hline & SPC & SCC & CC & LPC & PSC \\
\hline SPC & 1.0000 & -0.0439 & 0.8375 & 0.2177 & 0.8569 \\
SCC & & 1.0000 & -0.0207 & 0.0928 & 0.0143 \\
CC & & & 1.0000 & 0.0186 & 0.7368 \\
LPC & & & & 1.0000 & 0.1040 \\
PSC & & & & & 1.0000 \\
\hline
\end{tabular}

\subsection{Raw milk quality evaluation by conventional tests and TRF analysis}

\subsubsection{Conventional tests and raw milk quality}

Raw milk samples were evaluated as "high quality" or "low quality" (Appendix 2) by SPC, SCC, CC and LPC respectively according to the cutoffs in Table 5. The numbers and percentage of samples in high and low quality defined by each test were summarized in Table 8 . High quality milk samples defined by SPC were more common (64\%) than low quality milk. When evaluating with other tests, samples in high and low quality were more evenly distributed. The evaluation with combinations of different tests was not considered due to a very unbalanced distribution of milk samples in different quality (only $20 \%$ could be defined as "high quality").

Table 8. Summary of milk quality evaluation by SPC, SCC, CC and LPC.

\begin{tabular}{cccccccccc}
\hline & \multicolumn{2}{c}{ SPC } & \multicolumn{2}{c}{ SCC } & \multicolumn{2}{c}{ CC } & \multicolumn{2}{c}{ LPC } \\
\cline { 2 - 9 } & $\mathrm{n} *$ & Percent & $\mathrm{n}$ & Percent & $\mathrm{n}$ & Percent & $\mathrm{n}$ & Percent \\
\hline High quality & 23 & $64 \%$ & 19 & $53 \%$ & 19 & $53 \%$ & 18 & $50 \%$ \\
Low quality & 13 & $36 \%$ & 17 & $47 \%$ & 17 & $47 \%$ & 18 & $50 \%$ \\
Total & 36 & $100 \%$ & 36 & $100 \%$ & 36 & $100 \%$ & 36 & $100 \%$ \\
\hline
\end{tabular}

* "n" represents "the number of milk samples" 


\subsubsection{TRF analysis and raw milk quality}

There were 220 different TRF peaks generated by digestion of DpnII observed in total across all samples. The numbers of peaks obtained from each individual sample ranged from 11 (sample 12-158) to 39 (sample 5-179). When quality was determined by SPC or LPC, the numbers of TRF peaks from high quality milk were significantly higher than low quality milk at $\alpha=0.05$. Meanwhile, no significant difference of the numbers of TRF peaks was detected between high quality and low quality milk as determined by SCC or CC (Table 9).

Table 9. ANOVA results of numbers of TRF peaks (DpnII)

\begin{tabular}{lllc}
\hline & \multicolumn{2}{c}{ Mean of peaks } & \\
\cline { 2 - 3 } Tests & High quality & Low Quality & p-value \\
\hline SPC & $25.3 \pm 7.1$ & $20.1 \pm 5.8$ & 0.033 \\
SCC & $23.3 \pm 5.3$ & $23.3 \pm 8.8$ & 0.192 \\
CC & $24.7 \pm 7.9$ & $21.9 \pm 5.9$ & 0.232 \\
LPC & $25.8 \pm 6.7$ & $20.9 \pm 6.8$ & 0.036 \\
\hline
\end{tabular}

Comparing the abundance of different TRF peaks, when milk quality was determined by SPC, among all the TRF peaks, TRF peaks at $268 \mathrm{bp}, 270 \mathrm{bp}$ and $273 \mathrm{bp}$ had the greatest differences and higher abundance in low quality milk (Figure 2). When determined by LPC, TRF peaks at $168 \mathrm{bp}, 188 \mathrm{bp}$ and $273 \mathrm{bp}$ had the greatest differences. For $168 \mathrm{bp}$ and $273 \mathrm{bp}$, the average abundance was higher in low quality milk; however, for $188 \mathrm{bp}$, it appeared that the average abundance was higher in high quality milk (Figure 3). 


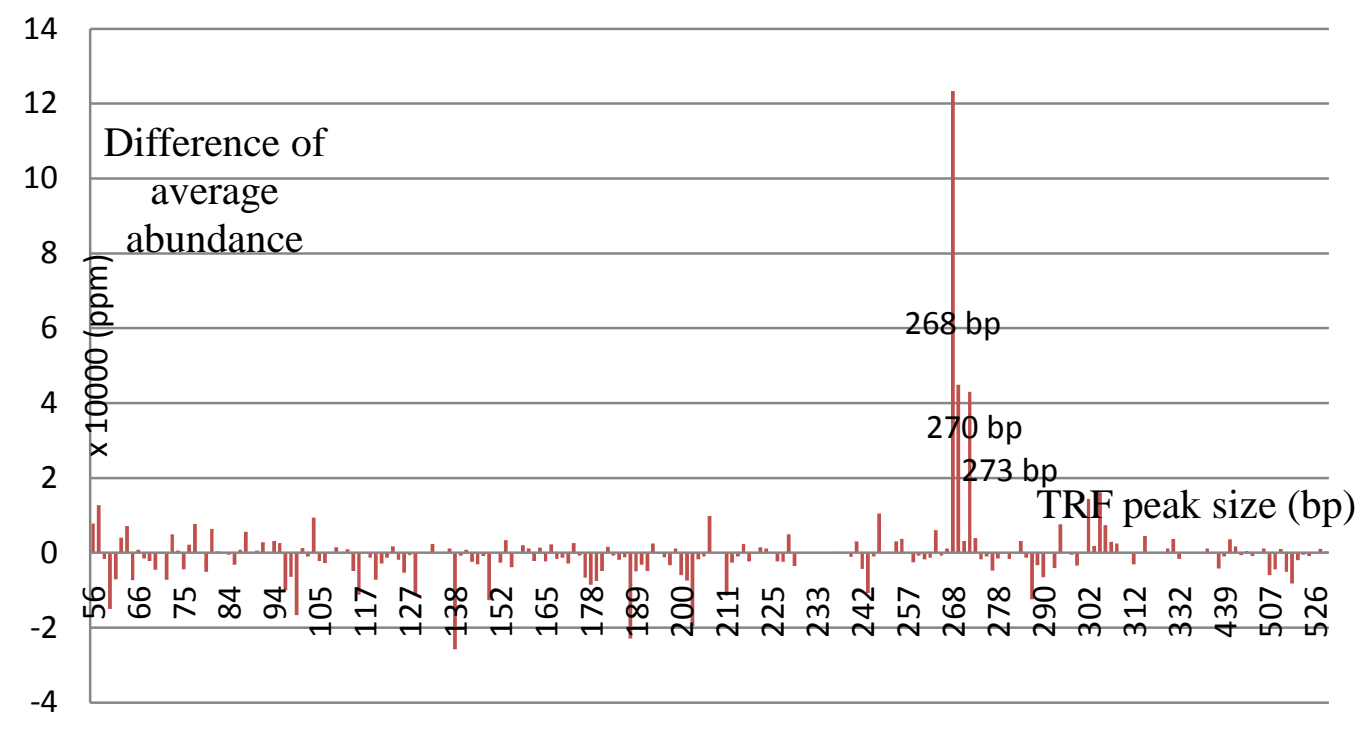

Figure 2. The differences of average abundance of TRF peaks ( $D p n I I)$ between low and high quality milk defined by SPC. For each peak, the difference of average abundance $=$ the average abundance from low quality milk - the average abundance from high quality milk.

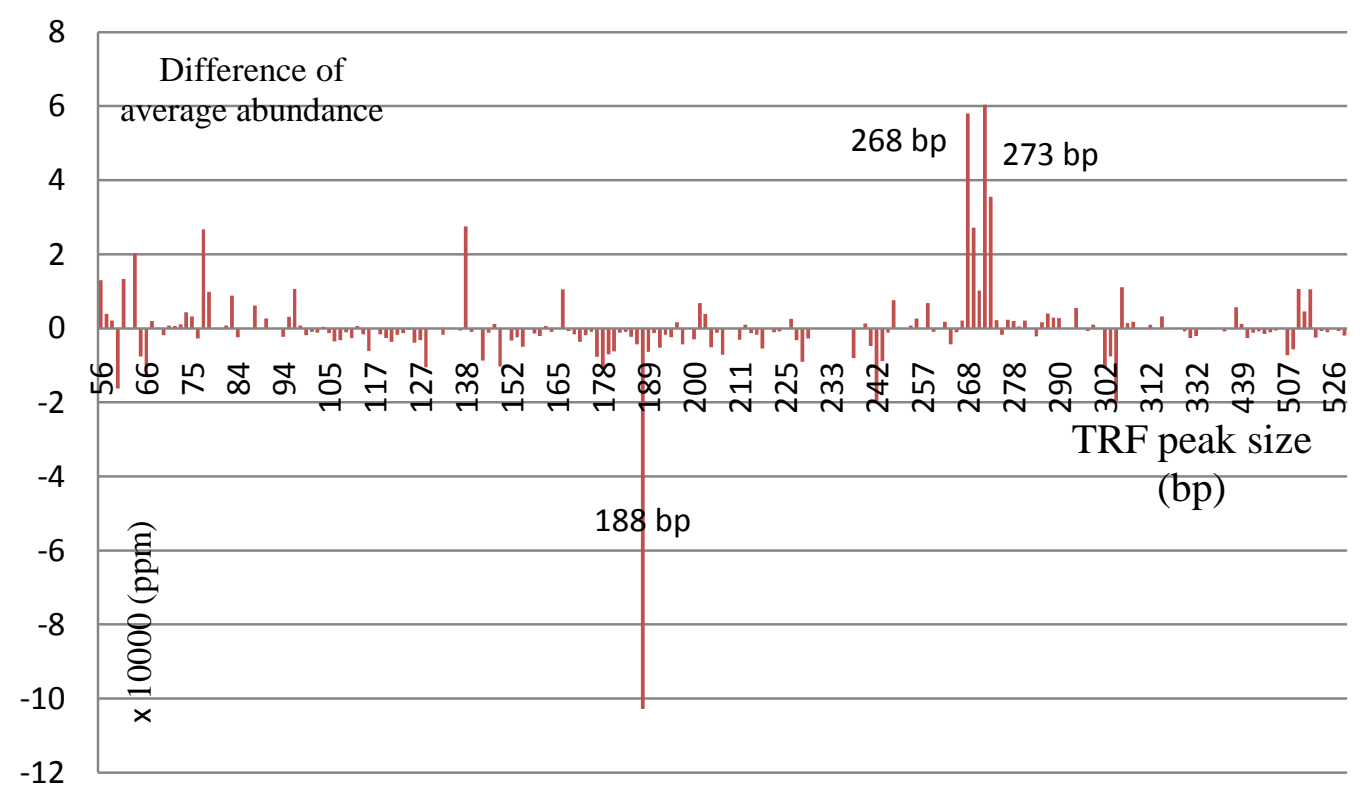

Figure 3. The differences of average abundance of TRF peaks (DpnII) between low and high quality milk defined by LPC. For each peak, the difference of average abundance $=$ the average abundance from low quality milk - the average abundance from high quality milk. 
Subsequently, NPMANOVA analyses were conducted on the normalized abundance of TRF peaks to detect the difference between TRF patterns derived from high and low quality milk samples. As determined by SPC or LPC, there was a significant difference between TRF patterns of high and low quality milk at $\alpha=0.05$ (p-value was 0.017 for SPC and 0.0186 for LPC). Meanwhile, no significant difference was found between TRF patterns of high and low quality milk as determined by $\mathrm{CC}(\mathrm{p}=0.1331)$ or $\mathrm{SCC}(\mathrm{p}=0.2056)$. SIMPER analysis revealed 15 TRF peaks contributing the most to the dissimilarity between samples in high and low quality determined by different tests (Table 10). Overall, the majority of peaks contributing the most to the dissimilarity were the same among milk samples evaluated by SPC or LPC. When milk quality was determined by SPC, TRF peaks at 268 bp contributed the most to the total dissimilarity. When determined by LPC, milk samples had 3 different TRF peaks (77 bp, 201 bp and $273 \mathrm{bp}$ ) and the contributor ranked in the first place was TRF peak at $188 \mathrm{bp}$ instead of 268 bp, which was ranked the second. 
Table 10. Fifteen TRF peaks contributing the most to the dissimilarity between high and low quality milk defined by SPC and LPC

\begin{tabular}{|c|c|c|c|c|c|c|c|}
\hline \multirow[t]{2}{*}{ Tests } & \multirow[t]{2}{*}{ Total Diss } & \multirow[t]{2}{*}{ TRF (bp) } & \multicolumn{2}{|c|}{ Average Abundance } & \multirow{2}{*}{ Av. Diss } & \multirow[t]{2}{*}{ Diss/SD } & \multirow{2}{*}{$\begin{array}{c}\text { Contribution } \\
\%\end{array}$} \\
\hline & & & Low quality & High quality & & & \\
\hline \multirow[t]{14}{*}{ SPC } & 80.1 & 268 & 151652.67 & 28327.08 & 2.16 & 1.25 & 2.7 \\
\hline & & 270 & 52333.42 & 7396.53 & 1.65 & 1.16 & 2.06 \\
\hline & & 274 & 40991.65 & 37120.63 & 1.63 & 1.04 & 2.03 \\
\hline & & 188 & 46311.31 & 69200.11 & 1.61 & 0.91 & 2.02 \\
\hline & & 242 & 21199.07 & 25510.41 & 1.42 & 1.27 & 1.78 \\
\hline & & 104 & 27588.46 & 18235.58 & 1.35 & 1.1 & 1.68 \\
\hline & & 63 & 30075.23 & 22932.34 & 1.32 & 0.95 & 1.65 \\
\hline & & 81 & 27475.25 & 21054.44 & 1.32 & 1.18 & 1.64 \\
\hline & & 61 & 8341.8 & 15432.08 & 1.2 & 1.03 & 1.5 \\
\hline & & 430 & 15903.17 & 20061.51 & 1.19 & 0.93 & 1.48 \\
\hline & & 202 & 36417.78 & 55973.22 & 1.19 & 1.01 & 1.48 \\
\hline & & 87 & 17134.13 & 11490.59 & 1.16 & 0.98 & 1.40 \\
\hline & & 75 & 10466.39 & 14841.55 & 1.12 & 0.98 & 1.40 \\
\hline & & 266 & 11054.12 & 9887.61 & 1.10 & 0.96 & 1.38 \\
\hline \multirow[t]{15}{*}{ LPC } & 80.41 & 188 & 9521.12 & 112348.3 & 2.03 & 1.07 & 2.52 \\
\hline & & 268 & 101862.1 & 43860.55 & 1.78 & 1.13 & 2.21 \\
\hline & & 274 & 56265.83 & 20771.17 & 1.67 & 1.03 & 2.07 \\
\hline & & 242 & 14015.84 & 33891.23 & 1.4 & 1.12 & 1.74 \\
\hline & & 270 & 37225.1 & 10022.38 & 1.33 & 1.02 & 1.66 \\
\hline & & 63 & 35613.21 & 15410.21 & 1.33 & 0.99 & 1.66 \\
\hline & & 104 & 21816.18 & 21409.83 & 1.31 & 1.12 & 1.63 \\
\hline & & 202 & 50851.73 & 46971.33 & 1.27 & 1.05 & 1.58 \\
\hline & & 81 & 23242.44 & 23503.69 & 1.24 & 1.15 & 1.54 \\
\hline & & 273 & 64121.54 & 3774.03 & 1.22 & 0.65 & 1.52 \\
\hline & & 61 & 19513.91 & 6229.49 & 1.18 & 1.08 & 1.47 \\
\hline & & 201 & 15677.75 & 8897.94 & 1.16 & 1.1 & 1.44 \\
\hline & & 430 & 21420.63 & 15699.14 & 1.15 & 0.92 & 1.43 \\
\hline & & 77 & 30189.69 & 3509.16 & 1.11 & 0.88 & 1.38 \\
\hline & & 75 & 14864.72 & 11658.54 & 1.11 & 1.01 & 1.38 \\
\hline
\end{tabular}

\subsection{Factors influencing milk quality}

\subsubsection{Analyses of conventional data}

The data set of conventional tests (Table 6) was analyzed by PCA first to visualize its internal structure. In Appendix 1, it was demonstrated that the first 2 principal components (PCs) 
consisted of $70 \%$ of the variation of the entire data set. Thus, they were selected for the following analysis. To visualize the dispersion of samples, a series of plots of the two PCs were conducted. The score plot (Figure 4) showed that the distribution of samples collected from wet days and dry days appeared to be separated by PC1 $(\mathrm{p}<0.05)$. Most of the samples collected on wet days had the PC1 $>0$ while those collected on dry days had PC1 $<0$. With regard to cow's apparent health, no clear separation was shown ( $p>0.05$ ) (Figure 5). The Biplot (Figure 6) provided more information about how the variables affected the sample distribution. The samples distributed in three main directions. The first one was composed of SCC and $\mathrm{pH}$, the second one was composed of SPC, CC and PSC and the last one was composed of LPC only.

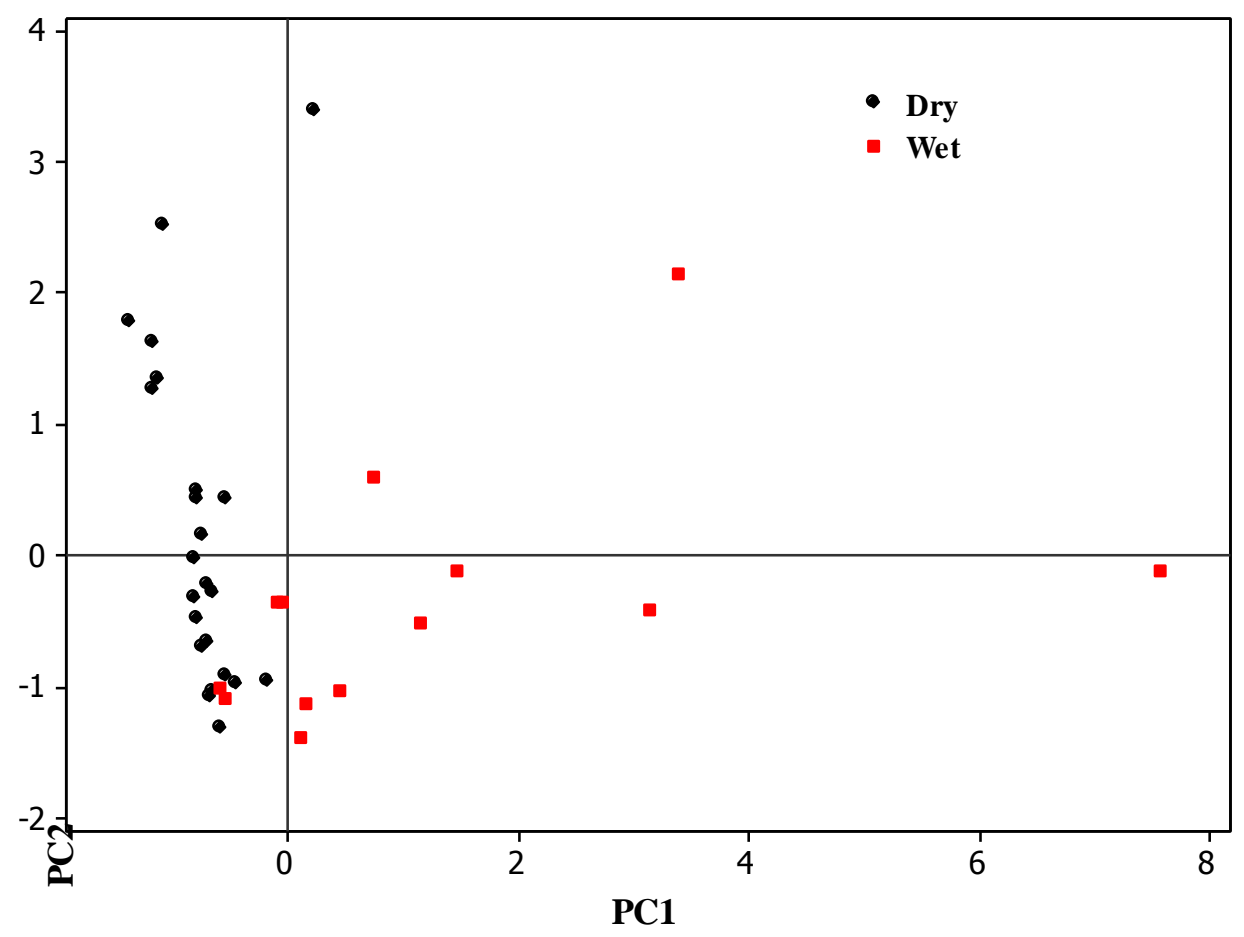

Figure 4. Score plot of the 1st and 2nd principal components from PCA of conventional testssamples grouped by rain event 


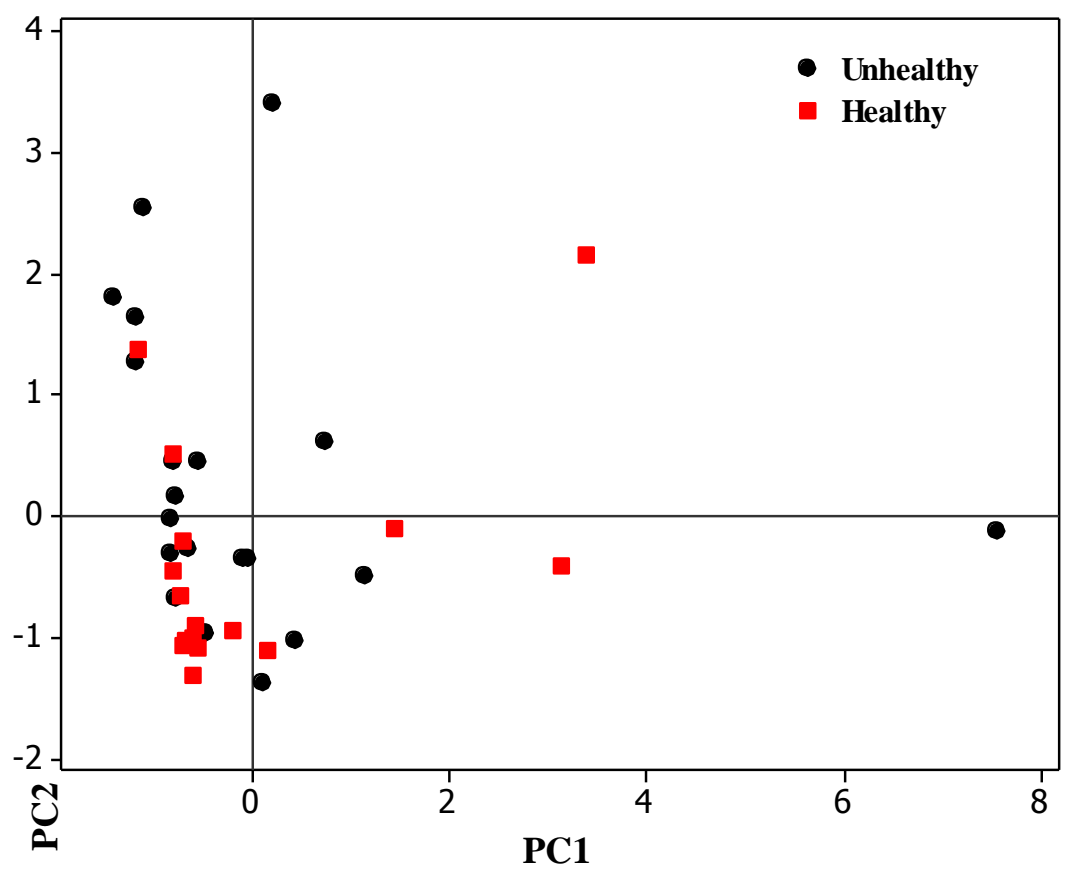

Figure 5. Score plot of the 1st and 2nd principal components from PCA of conventional testssamples grouped by cow's apparent health

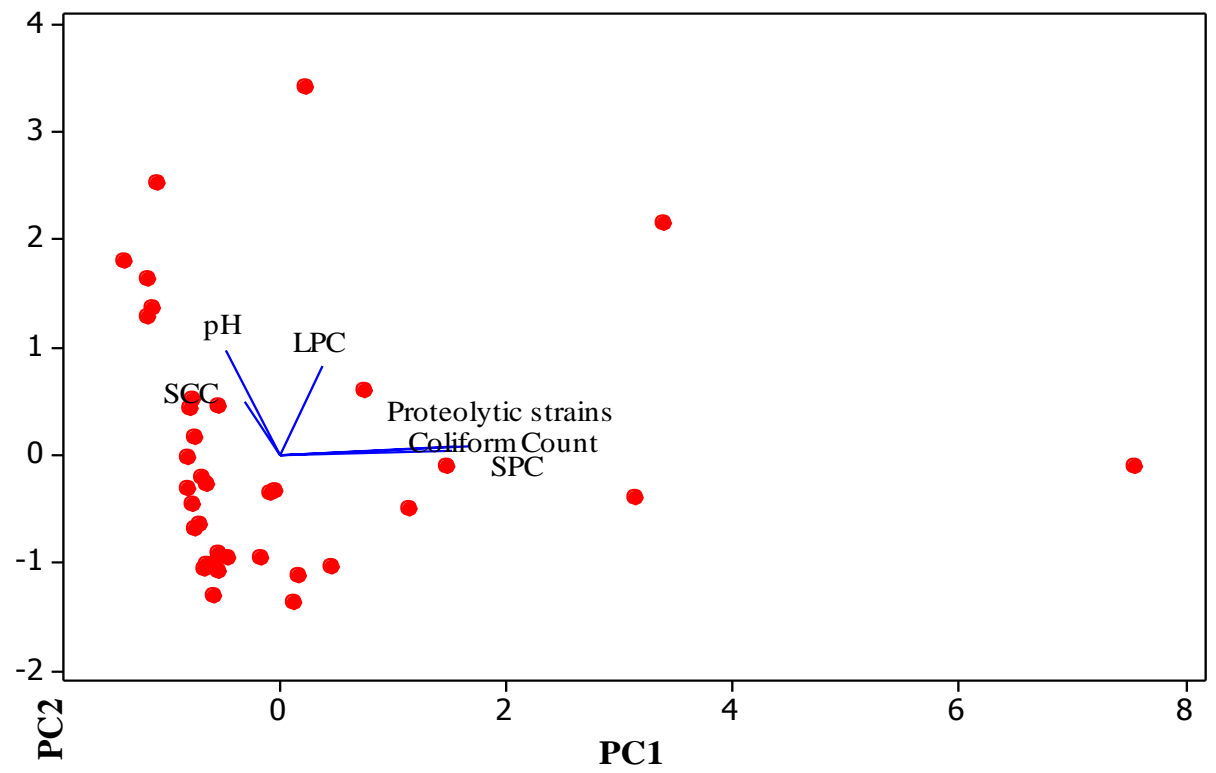

Figure 6. Biplot of the $1^{\text {st }}$ and $2^{\text {nd }}$ principal component from PCA of conventional tests 
MDS was used to complement the information provided by score plot. MDS can map the position of each sample by its distance from other points using an iterative algorithm that takes the multidimensional data of a similarity matrix and presents it in minimal dimensional space. MDS views high-dimensional data in a lower-dimensional plot. In the MDS plot, samples on the same level were circled to indicate groups. The more samples fall into the overlapped area, the more similarity the groups have. In the plot with grouping according to rain event, only a small number of samples were in the overlapped area. This implied a large dissimilarity between the two groups of samples (Figure 7). When grouped by cow's apparent health, most samples fell into the overlapped area and therefore there was no obvious difference between the apparent healthy and unhealthy groups (Figure 8).

PCA and MDS could reveal the influence of factors visually. However, neither of them was capable of providing a p-value to test whether the difference between groups was significant. 


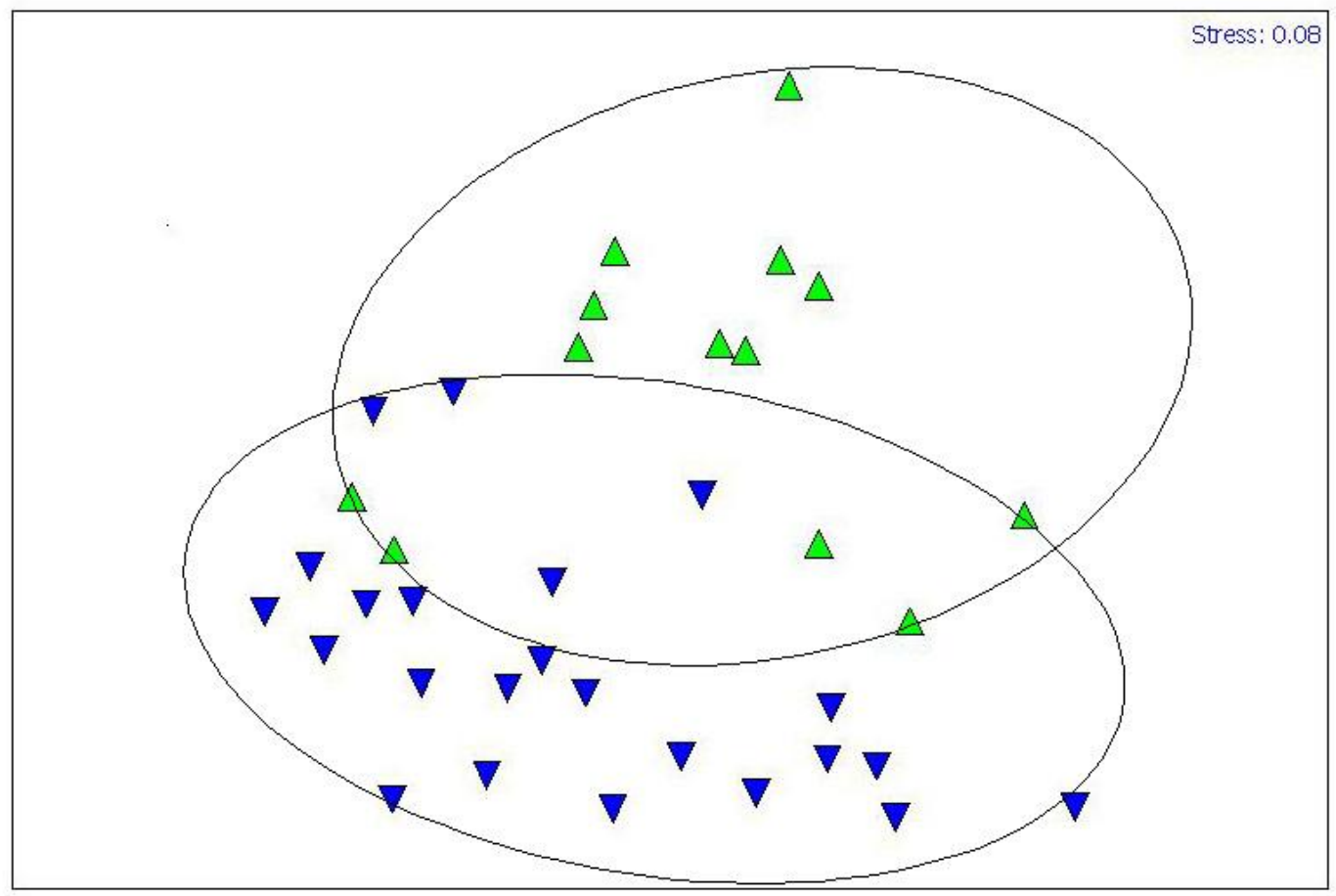

$\triangle$ Wet

₹ Dry

Figure 7. MDS plot of conventional tests data - Rain event

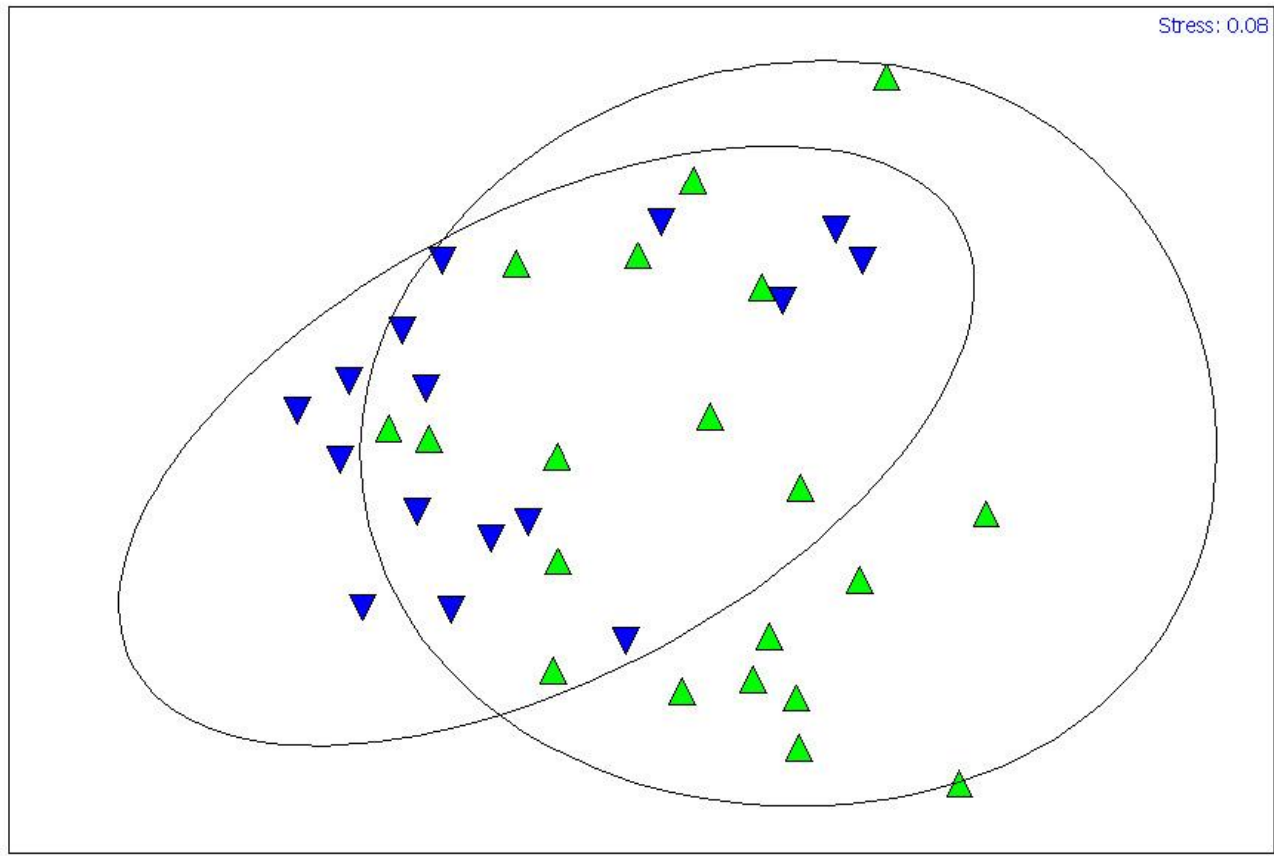

$\triangle$ Unhealthy

$\nabla$ Healthy

Figure 8. MDS plot of conventional tests data - cow's apparent health 
In order to obtain the p-values, MANOVA were conducted for the data of conventional tests.

The null hypothesis of MANOVA was that there was no difference among samples grouped by factors of rain event and cow's apparent health at $\alpha=0.01$ and each individual cow had no intrinsic effect on the variables. The MANOVA tests (Pillai) revealed a significant multivariate effect when samples were separated by rain event $(\mathrm{p}=0.006042)$ and cow's apparent health $(\mathrm{p}=0.007529)$. No interaction of the two factors was found as the $\mathrm{p}$-value was greater than 0.01 . Besides, the individual cows had no significantly intrinsic influence on the variables.

Table 11. MANOVA of conventional tests, rain event, cow's apparent health and individual cows.

\begin{tabular}{cccc}
\hline Factor & Df & Pillai F & p-value \\
\hline Rain event & 1 & 0.9676 & 0.006042 \\
Cow's apparent health & 1 & 0.9637 & 0.007529 \\
Rain event*Cow's apparent health & 1 & 0.4017 & 0.818821 \\
Cow & 23 & 4.5834 & 0.162399 \\
Residuals & 9 & & \\
\hline
\end{tabular}

Given the significant differences of the overall test, the univariate main effects were examined. Although this step ignored the fact that the variables may be intercorrelated and the ANOVAs did not take that into account, it was still useful to indicate the factors' effects on each variable. All the p-values were listed in Table 12.

Table 12. $\mathrm{p}$-values from ANOVA of pH, SPC, SCC, CC, LPC and PSC versus rain event, cow's apparent health status and individual cows ("*" is labeled where p-value $<0.01$ )

\begin{tabular}{ccccccc}
\hline Factor & \multicolumn{7}{c}{ p-value } \\
\cline { 2 - 7 } & $\mathrm{pH}$ & SPC & SCC & CC & LPC & PSC \\
Rain event & $0.000322^{*}$ & $1.323 \mathrm{e}-05^{*}$ & 0.098 & $2.735 \mathrm{e}-06^{*}$ & 0.1128 & $0.0002^{*}$ \\
Cow's apparent health & $0.009323^{*}$ & 0.03437 & $0.00012^{*}$ & 0.01195 & 0.6028 & 0.2246 \\
Rain event* cow's apparent health & 0.875861 & 0.23210 & 0.65713 & 0.44432 & 0.9456 & 0.7791 \\
Individual cow & $0.00827^{*}$ & 0.33868 & $0.00235^{*}$ & 0.10722 & 0.4921 & 0.6793 \\
\hline
\end{tabular}


At $\alpha=0.01$, rain event gave rise to the significant difference with the variables: $\mathrm{pH}, \mathrm{SPC}, \mathrm{CC}$ and PSC, whereas it had no significant influence on LPC and SCC. Significant differences in SCC were seen with cow's apparent health and across individual cows while cow's apparent health showed no significant influence on other bacteria counts, it did influence the $\mathrm{pH}$ significantly as is referred in the biplot of Figure 6.

\subsubsection{Analyses of TRF data}

In Table 13, the p-values suggested that rain event had significant influence on the TRF patterns generated by DpnII digestion, but cow's apparent health didn't show any significant influence. There was also no significant interaction between rain event and cow's apparent health on the TRF patterns. Individual cows had no significant influence one the TRF patterns as well.

Table 13. Output of NPMANOVA for TRF data by DpnII versus rain event, cow's apparent health and individual cows.

\begin{tabular}{llllll}
\hline & Df & Sums of Squares & Mean Squares & F. & P-value \\
\hline Rain event & 1 & 0.9827 & 0.98271 & 2.56045 & 0.0022 \\
Cow's apparent health & 1 & 0.4685 & 0.46853 & 1.22076 & 0.235 \\
Rain event*Cow's apparent health & 1 & 0.2889 & 0.28892 & 0.75277 & 0.7856 \\
Individual cow & 23 & 7.404 & 0.32191 & 0.83874 & 0.9417 \\
Residuals & 9 & 3.4542 & 0.3838 & & \\
Total & 35 & 12.5984 & & & \\
\hline
\end{tabular}

On the other side, the numbers of TRF peaks of each sample versus rain event and cow's apparent health were compared (Table 14). Samples collected on dry days had a significant higher number of peaks than samples collected on wet days $(\mathrm{p}=0.025)$. Comparing the average abundance of each TRF peak generated by DpnII, TRF peaks at 268 bp and 270 bp had much higher abundance in milk samples collected on wet days. No significant difference was seen in 
the numbers of peaks between samples collected from apparent healthy cows and unhealthy cows.

Table 14. ANOVA of TRF numbers of raw milk samples

\begin{tabular}{cccc}
\hline & p-value & \multicolumn{2}{c}{ Average number of peaks } \\
\hline Rain event & & Wet & Dry \\
& 0.025 & $20 \pm 1.824$ & $25 \pm 1.379$ \\
& & & \\
Cow apparent health & & Healthy & Unhealthy \\
Rain event*Cow apparent health & 0.092 & $20 \pm 1.693$ & $24 \pm 1.537$ \\
\hline
\end{tabular}

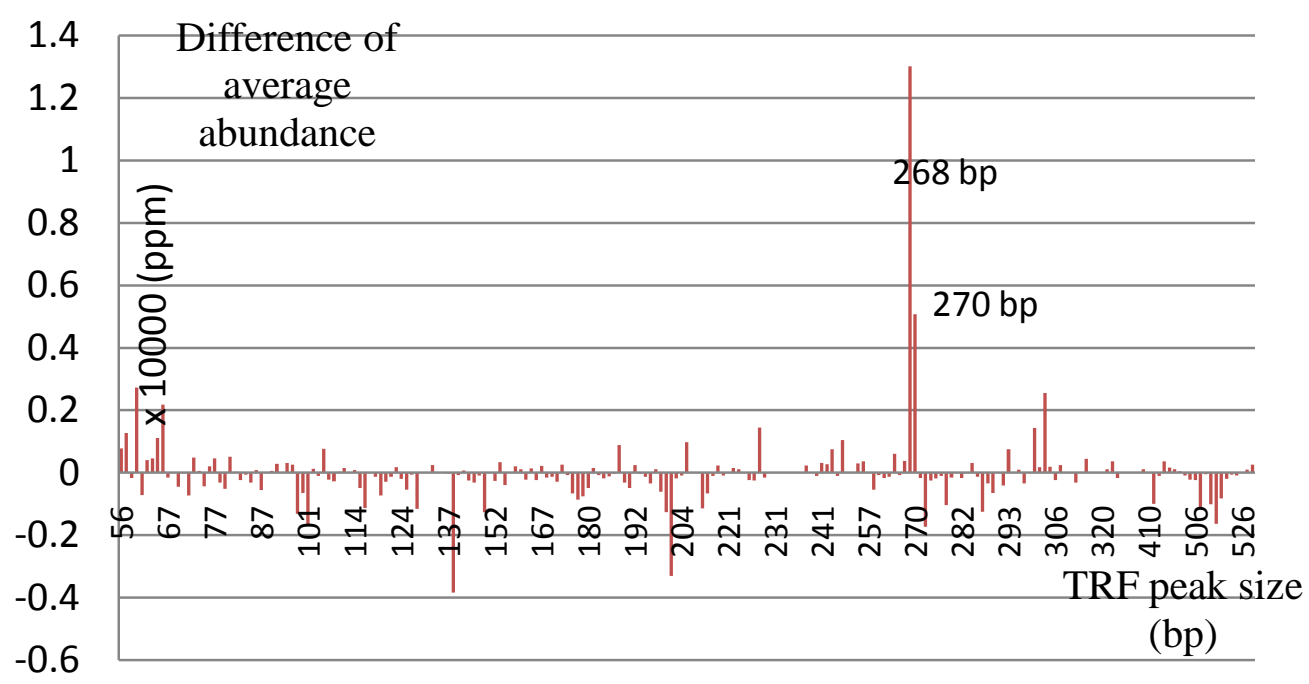

Figure 9. The differences of average abundance of TRF peaks (DpnII) between milk samples collected on wet and dry days. For each peak, the difference of average abundance $=$ the average abundance from milk samples collected on wet days - the average abundance from milk samples collected from dry days.

Between samples were collected in dry versus wet days, TRF peaks at $268 \mathrm{bp}$ with the average dissimilarity of $2.96 \%$ contributed the most to the total dissimilarity and had the highest 
abundance among all peaks. Other TRF peaks such as those at 188 bp, 202 bp, 242 bp, 270 bp, etc. were in the list of TRF peaks contributing the most to the total dissimilarity (Table 15).

Table 15. TRF peaks (DpnII) contributing the most to the dissimilarity between bacterial community associated with samples collected in wet and dry days

\begin{tabular}{cccccc}
\hline \multirow{2}{*}{ TRF (bp) } & \multicolumn{2}{c}{ Average Abundance } & Av. Diss & Diss/SD & Contribution \\
\cline { 2 - 3 } & Wet & Dry & & & $\%$ \\
\hline 268 & 156017 & 25860 & 2.41 & 1.39 & 2.96 \\
270 & 56010 & 5318 & 1.95 & 1.38 & 2.39 \\
188 & 60725 & 61052 & 1.67 & 0.98 & 2.05 \\
274 & 36943 & 39408 & 1.63 & 1.05 & 2 \\
242 & 25721 & 22954 & 1.53 & 1.35 & 1.88 \\
202 & 27791 & 60849 & 1.39 & 1.12 & 1.7 \\
81 & 26630 & 21531 & 1.38 & 1.27 & 1.69 \\
63 & 28476 & 23835 & 1.37 & 0.98 & 1.67 \\
104 & 26497 & 18852 & 1.36 & 1.12 & 1.66 \\
61 & 8341 & 15432 & 1.2 & 1.03 & 1.47 \\
430 & 12226 & 22139 & 1.16 & 0.91 & 1.43 \\
\hline
\end{tabular}




\subsection{Database matching}

Database matching was performed to identify the species that TRF peaks represented by combining the TRFs generated by DpnII and HaeIII. TRF peaks of high abundance and large contribution to the dissimilarity between groups of milk samples were selected to match the database from Genbank (Benson et al., 1998), since they were seen to discriminate samples in different quality (Table 16).

Table 16. Database matching of TRF peaks associated with raw milk quality $(b p=$ base pair)

\begin{tabular}{|c|c|c|c|c|}
\hline $\begin{array}{c}\text { Peak size }(D p n \mathrm{II}) \\
(\mathrm{bp})\end{array}$ & $\begin{array}{c}\text { Average } \\
\text { abundance }\end{array}$ & $\begin{array}{c}\text { peak size(HaeIII) } \\
\text { (bp) }\end{array}$ & $\begin{array}{c}\text { Average } \\
\text { abundance }\end{array}$ & Presumptive genus/species \\
\hline 61 & 12871 & $\begin{array}{c}307 \\
229-231 \\
224-225\end{array}$ & $\begin{array}{c}4031 \\
20720 \\
5865\end{array}$ & $\begin{array}{c}\text { Bacillus cereus } \\
\text { Bacillus mycoides } \\
\text { Pseudomonas putida }\end{array}$ \\
\hline 63 & 25512 & 225 & 3910 & $\begin{array}{c}\text { Alpha proteobacterium } \\
\text { Arthrobacter spp }\end{array}$ \\
\hline 75 & 13262 & $224-226$ & 6307 & Lactobacillus spp \\
\hline 81 & 23373 & $\begin{array}{c}127 \\
202-204 \\
224\end{array}$ & $\begin{array}{c}2431 \\
27826 \\
8864\end{array}$ & $\begin{array}{l}\text { Uncultured rumen bacterium } \\
\text { Arcocellaaquatica } \\
\text { Kineococcus-like bacterium } \\
\text { Pseudomonas marginalis }\end{array}$ \\
\hline 87 & 13529 & $\begin{array}{c}229-231 \\
228-230 \\
258 \\
226\end{array}$ & $\begin{array}{c}20720 \\
21718 \\
6713 \\
7193\end{array}$ & $\begin{array}{l}\text { Pseudomonas spp. } \\
\text { Staphylococcus spp. } \\
\text { Bacillus pumilus } \\
\text { Bacillus subtilis }\end{array}$ \\
\hline 98 & 10515 & 201-203 & 38772 & Uncultured cyanobacterium \\
\hline 104 & 21613 & 128 & 12090 & Pseudomonas spp \\
\hline 188 & 60935 & 68 & 47953 & $\begin{array}{l}\text { Streptococcus bovis } \\
\text { Pseudomonas stutzeri } \\
\text { Pseudomonas tolaasii }\end{array}$ \\
\hline
\end{tabular}


Continued Table 16

\begin{tabular}{|c|c|c|c|c|}
\hline 202 & 48912 & $\begin{array}{l}146 \\
226\end{array}$ & $\begin{array}{l}4313 \\
7193\end{array}$ & $\begin{array}{c}\text { Bacillus macroides } \\
\text { Paracoccus spp. }\end{array}$ \\
\hline 242 & 23954 & $\begin{array}{c}192-194 \\
227-228 \\
225 \\
71\end{array}$ & $\begin{array}{c}21830 \\
10539 \\
7193 \\
14450\end{array}$ & $\begin{array}{c}\text { Alpha proteobacterium } \\
\text { Bacillus.brevis } \\
\text { Gamma proteobacterium } \\
\text { Pseudomonas putida }\end{array}$ \\
\hline 266 & 10309 & 68 & 47953 & $\begin{array}{c}\text { E. coli } \\
\text { Enterococcus faecium }\end{array}$ \\
\hline 268 & 72861 & 197-199 & 7672 & $\begin{array}{l}\text { Pseudomonas spp. } \\
\text { Pseudomonas chlororaphis } \\
\text { Pseudomonas stutzeri }\end{array}$ \\
\hline 430 & 18560 & 233 & 32652 & Paenibacillus/Bacillus spp \\
\hline
\end{tabular}




\section{Chapter 5 Discussion}

\subsection{Correlations between conventional tests}

SPC and SCC have been routinely used for raw milk quality evaluation. However, they may not be adequate. To assess the predictive value of one conventional test for estimating the results of other ones, correlation coefficients between bacterial counts (SPC, CC, LPC and PSC) and SCC were calculated. The data showed that SPC was strongly correlated to CC and PSC (correlation coefficients $>0.8$ ), which means the total bacteria count in raw milk was strongly correlated to the population of coliforms and proteolytic bacteria, respectively. In a previous study by Boor (1998), raw milk quality of 800 samples from New York State was evaluated by SPC, CC and LPC. It was found that the correlations between tests were: SPC and LPC (0.3674), SPC and CC (0.4192), LPC and CC (0.1828). Although these correlations differed from those in our study, it is not surprising that correlations between tests varied among states because of differences between dairy environments including soil, humidity, temperature, etc. (Boor et al., 1998). With regards to SCC, it has been reported that there is an existing but unclear correlation between bacterial numbers and somatic cell counts (Marth and Steele, 2001). However, in this study, there was no correlation between SCC and bacterial counts (correlation coefficient <0.01).

Overall, the differences of correlation coefficients among studies suggested that one conventional test cannot be used to estimate other tests and that there is no reliable predictive value between results among conventional tests for the evaluation of raw milk quality (Elmoslemany et al., 2009). 


\subsection{Milk quality evaluation by TRF analysis}

Beginning with milk quality evaluation using conventional tests, TRF analysis was subsequently used to evaluate the microbial communities associated with high and low quality milk defined by conventional tests. When defined by SPC or LPC, high quality milk had a significantly higher number of TRF peaks than low quality milk. Considering TRF profiles represented the bacterial community in raw milk, it suggested that though the low quality milk had higher SPC or LPC, they had fewer different types of bacteria than those in high quality milk. It was reported that the microbial balance in raw milk may be critically impacted by even the presence of one single different species (Lafarge et al., 2004), so the high SPC/LPC in low quality milk may be caused by large population of one or a few predominant genus/species. It indicated that the population distribution of bacteria in raw milk was skewed. Figure 2 and Figure 3 showed a few TRF peaks such as TRF peaks at $188 \mathrm{bp}, 268 \mathrm{bp}, 270 \mathrm{bp}$ and $273 \mathrm{bp}$ had much higher differences between high and low quality groups than any other peaks.

When defined by SPC, NPMANOVA of TRF data (DpnII) indicated there was a significant difference between the bacterial communities associated with high versus low quality milk. According to the results of SIMPER (Table 2), TRF peak at 268 bp seemed to be the most important one among all the TRF peaks because it contributed the highest percentage $(2.7 \%)$ to the dissimilarity. In addition, low quality milk had the highest average abundance of this peak. Thus, it implied that the genus/species represented by this peak were the key bacterial groups strongly associated with low quality milk. Hence, TRF peak at $268 \mathrm{bp}$ could be indicative of milk quality when evaluated by SPC: high quality milk is associated with low abundance of this peak while low quality milk with high abundance. After database matching, TRF peak at $268 \mathrm{bp}$ 
represented some species of Pseudomonas, including $P$. chlororaphis and $P$. stutzeri. In addition to $268 \mathrm{bp}$, TRF peaks at other sizes, $61 \mathrm{bp}, 81 \mathrm{bp}, 87 \mathrm{bp}, 104 \mathrm{bp}, 188 \mathrm{bp}$ and $242 \mathrm{bp}$, represented species of Pseudomonas as well. Many studies have reported that Pseudomonas spp. are one of the predominant groups in microbial community in raw milk and important contributors to the spoilage of conventionally pasteurized fluid milk products (Lafarge et al., 2004, Wiedmann et al., 2000). For example, P.chororaphis is a known proteolytic species that is able to cause spoilage (Nicodème et al., 2005). P. putida, which is generally isolated from fresh water, produce small but significant quantities of ethyl esters, resulting in off-odor defect of the tainted milk (Whitfield et al., 2001). With the capability of detecting the genus/species of microorganisms in raw milk, TRF peaks could be used to predict the occurrence of quality defects in raw milk.

When defined by LPC, TRF profiles between high and low quality milk showed significant differences as well. The peak contributing the most to the dissimilarity between profiles derived from high and low quality milk was at $188 \mathrm{bp}$. The average abundance of this peak in the profile of low quality milk was lower than that of high quality milk. It indicated that the species represented by this peak ( $P$. stutzeri/P. tolaasii) have higher average abundance in high quality milk than that in low quality milk. Since most of the Pseudomonas were destroyed during pasteurization (Sørhaug and Stepaniak, 1997), TRF peak at 188 bp cannot impact LPC significantly although it had a large difference in raw milk before pasteurization because LPC tests the population of thermoduric bacteria. Since the abundance of TRF peaks is relative, in this case, the relatively high abundance of this peak was likely caused by the low prevalence of other peaks representing Pseudomonas species, such as $268 \mathrm{bp}$. On the other hand, thermoduric bacteria were detected via TRF analysis. This is expected because the population of spores in raw milk is usually not less than 100 spores/ml (Crielly et al., 1994) which is higher than the 
DNA detection limit (100 spores/ml). One of the most important thermoduric groups associated with milk quality is Bacillus. TRF peaks representing Bacillus and related species included those at $61 \mathrm{bp}$ and $430 \mathrm{bp}$. It corresponded to the results of LPC that their average abundance in low quality milk was higher than that in high quality milk. This suggested that TRF analysis is capable of detecting spores in raw milk with acceptable sensitivity.

When SCC or CC was used to defined milk quality, there was no significant difference between the microbial communities associated with high and low quality milk. For SCC, it suggested that SCC had no significant influence on bacterial community. High SCC is usually caused by the cow's udder inflammation that is not always associated with bacteria (Marth and Steele, 2001), and other conventional tests also showed no correlation with SCC. For CC, the coliforms accounted for an extremely small proportion of the total bacterial community so that they could not impact the whole bacterial community significantly. According to the conventional tests, coliform groups accounted for approximately $1 / 1000$ of the total bacteria count. In this case, it was not surprising that the increased coliform population could not significantly influence the microbial communities. With regards to the peaks representing coliforms, TRF peak at $266 \mathrm{bp}$ was the most relevant peak that represented E. coli and Enterococcus faecium. Its average abundance in low quality milk was higher than that of high quality milk, which corresponded to the results of CC. Hence, when the microbial communities have no significant difference, the investigation of individual TRF peaks associated are still valuable for quality evaluation.

SIMER analysis indicated that some TRF peaks, for example, 188 bp, 242 bp, 268 bp, 430 bp, etc. consistently showed up among the peaks contributing the most to dissimilarity in TRF profiles regardless of quality evaluation as defined by different conventional tests. This implies that the genus/species they presented were predominant in the microbial community associated 
with raw milk. The abundance of those genus/species can play a significant role in raw milk quality. Therefore, SIMPER can be a valuable tool for TRF data analysis and milk quality evaluation.

Comparing the abundance of TRF peaks associated with raw milk, it was found that for some TRF peaks, the abundance was higher in high quality milk than low quality milk. This could partially explain the spoilage of "high quality milk" evaluated by conventional tests. Although high quality milk has low population of total bacteria, the spoilage causing bacteria may account for a large proportion of total bacteria, exceed the threshold of spoilage and cause quality defects. Particularly, when the population distribution was highly skewed, conventional tests cannot provide sufficient information on the change of the bacterial community structure. Thus, TRF analysis can be a complementation by providing more information of the composition of microbial community and improve milk quality evaluation.

\subsection{Complementation between TRF analysis and conventional tests}

TRF analysis is a valuable tool for investigating complex bacterial communities in raw milk, which makes it a useful tool for milk quality evaluation. TRF analysis had several advantages as a complementation of conventional tests.

First, TRF analysis is capable of providing comprehensive understanding of the bacterial community structure in raw milk. Conventional tests can only provide limited information of the composition of the bacterial community. Tests such as CC and LPC can determine the population of coliforms and thermoduric bacteria in a bacterial community, but they cannot provide specific information about the genus/species identities. Nevertheless, TRF analysis is able to yields the information on the genus/species in a microbial community. In this study, it 
indicated the predominant species associated with raw milk quality. With the knowledge of the bacterial community structure, corresponding countermeasures can be taken to ensure milk quality. For instance, current pasteurization is applied to ensure most of the bacteria in raw milk are destroyed regardless of the composition of how bacterial community varies in raw milk. With the application of TRF analysis, the time and temperature of pasteurization could be modified according to the bacterial community structure of raw milk via computer-based program to reach the highest efficiency of quality assurance and energy consumption. Simply, with the input of microbial composition of bacterial community, the computer-based program will provide the most effective pasteurization scheme.

Second, TRF analysis can reveal the presence of non-culturable bacteria in raw milk. It is known that not all microorganisms in raw milk are culturable (Wegmuller et al., 1993) as some undergo VBNC state. Microorganisms reported to exist in a VBNC state and present in milk include Pseudomonas putida, Enterobacter, Campylobacter jejuni, Bacillus cereus and E. coli (Rowan, 2004), some of which can cause milk spoilage, such as $P$. putida (Whitfield et al., 2001). Most conventional bacterial counts applied in dairy industry are culture-dependent, which determined bacteria that are grown on particular media, so bacteria in VBNC state cannot be detected. Thus, even if the milk is defined as high quality by conventional tests, it is possible that milk spoilage is caused by the presence of undetected VBNC bacteria. As a DNA-based culture-independent method, TRF analysis is capable of detecting both the culturable and non-culturable bacteria via 16S rDNA. Using TRF analysis, milk quality evaluation will be improved with the knowledge of VBNC bacteria.

In addition, TRF analysis generally does not bring a heavy burden to the quality control procedure already in place. Typically, conventional tests require two or more days of cultivation. 
TRF analysis carried out in this study required a total of two workdays, which means the conventional tests and TRF analysis can be accomplished simultaneously. Moreover, frozen milk samples may be used for TRF analysis, which will facilitate the tests of a number of samples in one experiment and save time (Rademaker et al., 2005). Compared to other methods that are used to analyze the composition of microbial communities, the total cost of TRF analysis is relatively low. For instance, the total cost to obtain a TRF profile is about $\$ 3.8$ per sample while the cost of 16S rDNA cloning is about $\$ 5$ per sample (Tanaka et al., 2010).

Although TRF analysis is useful for raw milk quality evaluation, there are some difficulties to resolve. First, TRF analysis is limited by the biases inherent to any DNA- and PCR- based investigation including DNA extraction, PCR and electrophoresis. However, this is not a disadvantage specific to TRF analysis because almost all other community analysis methods commonly used have the same problem (Kitts, 2001). Second, some TRF peaks may not be represented in the database if the existing database is not complete. To solve this problem, researchers may need to build clone libraries that can be analyzed by TRF analysis and then sequenced for identification to keep the database updated. Third, TRF analysis may detect nonviable bacteria in raw milk because DNA derived from non-viable but intact cells in raw milk samples can be extracted and subject to the TRF analysis. However, the non-viable bacteria consists of only a very small proportion of the total bacteria population in raw milk (Knaysi and Ford, 1938). Morever, since large numbers of bacteria in raw milk give evidence of unsanitary conditions, no matter whether the cells are viable, the method it still has its value as a quality control procedure to indicate the source of contamination. On the other hand, conventional tests can be a partial complement to TRF analysis as well because they can detect the population of viable bacteria in raw milk. 
Since both conventional tests and TRF analysis have their own drawbacks, each method shows a partially biased view of the actual situation of bacterial communities in raw milk (Rademaker et al., 2005). Conventional tests have shown their value for milk quality evaluation in the past decades. When combined with TRF analysis, limitations are decreased and the accuracy of milk quality evaluation can be enhanced.

\subsection{Factors influencing raw milk quality}

Poor quality milk is commonly caused by microorganisms from contamination sources, including the dairy environment, cow's udder and milking equipment (Murphy and Boor, 2000). In this study, since milking equipment had been sterilized, the main sources of microorganisms would be the dairy environment and cow's udder. Rain event is an important factor that can influence the dairy environment while cow's apparent health can have a direct influence on the udders. In addition, raw milk samples were collected from different individual cows, thus the influence of individual cows on the raw milk quality needed to be taken into account. Influence of these factors on milk quality was investigated by both conventional tests and TRF analysis. With regards to the conventional tests, the variables (pH, SPC, SCC, CC, LPC and PSC) were not totally independent since strong correlations were shown between some of them. Therefore, the statistics based on the assumption of independence was not suitable to analyze the data. In general, MANOVA showed that there was significant difference of results of conventional tests conducted on raw milk samples collected from dry and wet days. PCA and MDS showed that samples appeared as separate clusters when they were grouped according to the factor of "rain event". ANOVA of each variable was followed in order to investigate how each variable affected the overall results of MANOVA. It revealed that wet days led to a significant increase of $\mathrm{pH}$, 
SPC, CC and PSC, which means the wet days caused the population of total bacteria, coliforms and proteolytic bacteria increased. Rain event showed no significant influence on LPC, which indicated that there was no significant difference with the count of thermoduric (pasteurizationresistant) bacteria between the raw milk samples collected on wet and dry days. Although the total bacteria count increased greatly on wet days, thermoduric bacteria stayed at the same level, so the increase of total bacteria was due to non-thermoduric bacteria such as Pseudomonas spp. To sum up, raw milk samples collected in wet days harbored significantly higher level of coliform, proteolytic bacteria and non-thermoduric bacteria than in dry days and these groups collectively resulted in a higher level of total bacteria (determined by SPC). Microorganisms are ubiquitous the dairy environment. Cow's teats become dirty when contacting with the dairy environment. Microorganisms in the dirt could be transmitted to the raw milk directly (Christiansson et al., 1999). Generally, contamination with psychrotrophic microflora has been associated with bedding material, untreated water, soil, and vegetation; coliform contamination with soil and spore-formers with bedding material and soil (Marth and Steele, 2001). On wet days, the contamination of microorganisms was aggregated as teat dirtiness was increased by rain. However, if the teat cleansing was not improved, the population of microorganisms in raw milk could be increased dramatically. Hence, this was likely the main reason leading to the increase of SPC, CC and PSC.

On the aspect of TRF analysis, rain event had significant influence on the TRF profiles generated by $D p n I I$. It suggested that the bacteria community structure of raw milk was dependent on rain event. Comparing the numbers of TRF peaks from raw milk samples collected on dry days and wet days, the samples on wet days yielded less TRF peaks than those on dry days but they harbored higher levels of bacterial counts. This implied that the increase of total bacteria counts 
on wet days was caused by an increase of few predominant types instead of an increase of all members in the microbial community (Kitts, 2001). In terms of the contribution to the dissimilarity between microbial communities, TRF peaks at $268 \mathrm{bp}$, representing Pseudomonas spp, contributed the most and had the largest abundance in the microbial community. Moreover, the abundance of this peak in samples from wet days was seven times higher than that from dry days. TRF peaks contributing the most to the dissimilarity between raw milk in high and low quality defined by conventional tests were found contributing the most to the dissimilarity between bacterial communities associated with samples collected on wet/dry days as well. In addition, 11 out of 13 samples collected in wet days harbored SPC of greater than 50,000 cfu/ml. Therefore, we speculated that rain event was the factor causing poor quality with raw milk via increasing the population of predominant genus/species, such as Pseudomonas.

In summary, both the conventional tests and TRF analysis suggested that rain event was the main factor that influenced milk quality significantly in terms of bacterial count and composition of microbial community in raw milk and TRF analysis suggested that rain increases the population of Pseudomonas.

On the other hand, cow's apparent health did not have the same degree of impact compared to the rain event. Referring to the ANOVA, it had no significant influence on the bacteria counts according to (SPC, CC, LPC and PSC). Instead, it had significant influence on SCC and pH. This was expected because SCC was commonly used as the indicator of cow's apparent health. Cows with udder disease usually harbor high somatic cell count. In addition, disease in cow's udder can result in pH change in milk (Fenlon et al., 1995). Comparing SCC and cow's apparent health, it was found that the apparent "unhealthy" cows did not always yield high SCC while the 
"healthy" cows did not always yield low SCC as well. TRF data analysis also demonstrated that cow's apparent health had no significant difference on the microbial community.

Given all above, it was clear that wet weather could result in significantly higher level of bacteria in raw milk and had significant influence on the composition of microbial community. On the contrary, the cow's apparent health had no significant impact on bacterial counts. Considering that the high bacterial count of raw milk collected from wet days were likely caused by the increase of dirtiness of cow's teat, it was important and advisable for the dairy farmers to reinforce the cleaning process before milking in order to ensure the quality of raw milk especially during wet days (Christiansson et al., 1999). 


\section{Chapter 6 Further research}

The ultimate goal of this study is to enhance quality of raw milk and milk products by improving quality evaluation system. Our work suggested that molecular methods are promising for this objective and provided a basis for future studies. It also raises some new questions. In this circumstance, a series of studies can be implemented in the future to improve this project.

An ideal milk quality evaluation system in the future will feature automation, rapidness and high efficiency, which can remarkably reduce the cost of time, labor and expense of quality evaluation. This system will be able to picture the microbial community and provide a scheme for pasteurization with the highest efficiency and energy savings after a raw milk sample is input. To this end, some of the quality evaluation procedures need further optimization. For example, since DNA extraction cost almost half of testing time, the development of a faster and effective DNA extraction method, such as a commercial kit, is very essential. Moreover, considering that the abundance of bacteria from TRF analysis is a standardized relative value, other experiments can be introduced to estimate the absolute abundance of bacteria in raw milk in order to complete the picture of microbial communities. With the awareness of total bacterial population, the population of each species detected by TRF analysis can be calculated. Other than culturedependent methods (such as SPC), direct microscopic count and flow cytometry offer rapid techniques for determining the population of bacteria in raw milk including VBNC bacteria (Veal et al., 2000, Wehr and Frank, 2004).

This study proved the feasibility of molecular methods for evaluating microbial quality of raw milk with high efficiency and low cost. With the rapid development, new advanced methods can 
be employed in the future. For instance, the next generation of DNA sequencing technologies feature high throughput and rapidness for analysis of microbial communities (Shendure and Ji, 2008). One of the new technologies, pyrosequencing, has been used to investigate microbial communities associated with food samples. Using this method, massive parallel pyrosequencing (more than 300,000 sequences) can be determined simultaneously, and it does not require cloning of the samples, thus eliminating many of the problems associated with this step of metagenomic methods. A highly variable region of the $16 \mathrm{~S}$ rRNA gene is amplified using primers that target adjacent conserved regions, followed by direct sequencing of individual PCR products (Humblot and Guyot, 2009). This method would allow analysis of enough samples taken at regular times, generating in the same run an overall view of community structure in raw milk and significantly increase the efficiency of quality evaluation.

On the other hand, since the molecular methods cannot avoid the intrinsic shortcomings such as biases inherent to any DNA- and PCR- based investigation, it is significant to introduce alternative methods in the long run. Technologies of membrane filtration are gaining popularity in dairy applications. Among them, microfiltration (MF) offers an alternative to traditional microbiological methods. It can reduce the amount of bacteria and spores without affecting the taste of the milk and provide longer shelf life than pasteurization. However, although membrane filtration removes bacteria in raw milk, it cannot remove the spoilage-causing enzymes, thus it cannot completely solve all the quality problems. Besides, there are some limitations for the application of this technology, too. Fouling control and bacteria retention are still need to be improved and its cost is much higher than heat treatment (Saboyainsta and Maubois, 2000). In this case, studies on microbial communities are still necessary and valuable. 


\section{Chapter 7 Conclusions}

It can be concluded from this study that TRF analysis provides a relatively rapid and low-cost technique for studying the microbial community in raw milk and complementing conventional tests of raw milk quality evaluation. The combination of TRF analysis and conventional tests can provide a more comprehensive picture of the microbial community than either of them individually. Knowledge of the occurrence of certain genus/species of microorganisms in raw milk can aid in improving the milk quality evaluating system. In addition, TRF analysis and conventional tests may be used to analyze the factors affecting the microbial community with applicable statistical tools. 


\section{References}

Anderson, M. 2001. A new method for non-parametric multivariate analysis of variance. Austral Ecology 26(1):32-46.

Anonymous. 2009. Pasteurized Milk Ordinance 2009. U.S. Department of Health and Human

Services, Public Health Service, Food and Drug Administration.

Anonymous. 2010a. California Milk Standards.

Anonymous. 2010b. San Luis Obispo Polytech, California (047851): Period of Record Monthly

Climate Summary.

Barbano, D. M., Y. Ma, and M. V. Santos. 2006. Influence of raw milk quality on fluid milk shelf life. J Dairy Sci 89 Suppl 1:E15-19.

Belgrader, P., D. Hansford, G. Kovacs, K. Venkateswaran, R. Mariella Jr, F. Milanovich, S.

Nasarabadi, M. Okuzumi, F. Pourahmadi, and M. Northrup. 1999. A minisonicator to rapidly disrupt bacterial spores for DNA analysis. Anal. Chem 71(19):4232-4236.

Benson, D., M. Boguski, D. Lipman, J. Ostell, and B. Ouellette. 1998. GenBank. Nucleic Acids Res 26(1):1.

Boor, K. J., D. P. Brown, S. C. Murphy, S. M. Kozlowski, and D. K. Bandler. 1998. Microbiological and chemical quality of raw milk in New York State. J Dairy Sci 81(6):17431748.

Christiansson, A., J. Bertilsson, and B. Svensson. 1999. Bacillus cereus spores in raw milk: factors affecting the contamination of milk during the grazing period. Journal of Dairy Science 82(2):305-314.

Clarke, K. 1993. Non parametric multivariate analyses of changes in community structure. Australian Journal of Ecology 18(1):117-143.

Clement, B., L. Kehl, K. DeBord, and C. Kitts. 1998. Terminal restriction fragment patterns (TRFPs), a rapid, PCR-based method for the comparison of complex bacterial communities. J Microbiol Meth 31(3):135-142.

Coppola, S., G. Blaiotta, D. Ercolini, and G. Moschetti. 2001. Molecular evaluation of microbial diversity occurring in different types of Mozzarella cheese. J Appl Microbiol 90(3):414-420. Crielly, E., N. Logan, and A. Anderton. 1994. Studies on the Bacillus flora of milk and milk products. Journal of Applied Microbiology 77(3):256-263.

Danovaro, R., G. M. Luna, A. Dell'anno, and B. Pietrangeli. 2006. Comparison of two fingerprinting techniques, terminal restriction fragment length polymorphism and automated ribosomal intergenic spacer analysis, for determination of bacterial diversity in aquatic environments. Appl Environ Microbiol 72(9):5982-5989.

De Jonghe, V., A. Coorevits, J. De Block, E. Van Coillie, K. Grijspeerdt, L. Herman, P. De Vos, and M. Heyndrickx. 2010. Toxinogenic and spoilage potential of aerobic spore-formers isolated from raw milk. Int J Food Microbiol 136(3):318-325.

Delbes, C., L. Ali-Mandjee, and M. Montel. 2007. Monitoring bacterial communities in raw milk and cheese by culture-dependent and-independent 16S rRNA gene-based analyses. Appl Environ Microb. 
Dunbar, J., L. O. Ticknor, and C. R. Kuske. 2001. Phylogenetic specificity and reproducibility and new method for analysis of terminal restriction fragment profiles of 16S rRNA genes from bacterial communities. Appl Environ Microb 67(1):190-197.

Duthoit, F., J. Godon, and M. Montel. 2003. Bacterial community dynamics during production of registered designation of origin Salers cheese as evaluated by $16 \mathrm{~S}$ rRNA gene single-strand conformation polymorphism analysis. Appl Environ Microb 69(7):3840.

Elmoslemany, A., G. Keefe, I. Dohoo, and R. Dingwell. 2009. Microbiological quality of bulk tank raw milk in Prince Edward Island dairy herds. Journal of Dairy Science 92(9):4239-4248. Ercolini, D. 2004. PCR-DGGE fingerprinting: novel strategies for detection of microbes in food. J Microbiol Meth 56(3):297-314.

Fenlon, D. R., D. N. Logue, J. Gunn, and J. Wilson. 1995. A study of mastitis bacteria and herd management practices to identify their relationship to high somatic cell counts in bulk tank milk. Br Vet J 151(1):17-25.

Griffiths, M., J. Phillips, and D. Muir. 1981. Thermostability of proteases and lipases from a number of species of psychrotrophic bacteria of dairy origin. Journal of Applied Microbiology 50(2):289-303.

Gruntzig, B., B. Stres, H. Ayala del Rio, and J. Tiedje. 2002. Improved protocol for T-RFLP by capillary electrophoresis. Center for Microbial Ecology, Michigan State University, East Lansing, Michigan. .

Gunasekera, T., D. Veal, and P. Attfield. 2003. Potential for broad applications of flow cytometry and fluorescence techniques in microbiological and somatic cell analyses of milk. International journal of food microbiology 85(3):269-279.

Hantsis-Zacharov, E. and M. Halpern. 2007. Culturable psychrotrophic bacterial communities in raw milk and their proteolytic and lipolytic traits. Appl Environ Microbiol 73(22):7162-7168. Hengstmann, U., K. Chin, P. Janssen, and W. Liesack. 1999. Comparative phylogenetic assignment of environmental sequences of genes encoding 16S rRNA and numerically abundant culturable bacteria from an anoxic rice paddy soil. Appl Environ Microb 65(11):5050. Hertl, J. A., Y. T. Groehn, J. D. G. Leach, D. Bar, G. J. Bennett, R. N. Gonzalez, B. J. Rauch, F. L. Welcome, L. W. Tauer, and Y. H. Schukken. 2010. Effects of clinical mastitis caused by gram-positive and gram-negative bacteria and other organisms on the probability of conception in New York State Holstein dairy cows. Journal of Dairy Science 93(4):1551-1560.

Huck, J. R., B. H. Hammond, S. C. Murphy, N. H. Woodcock, and K. J. Boor. 2007. Tracking spore-forming bacterial contaminants in fluid milk-processing systems. J Dairy Sci 90(10):48724883.

Huck, J. R., M. Sonnen, and K. J. Boor. 2008. Tracking heat-resistant, cold-thriving fluid milk spoilage bacteria from farm to packaged product. J Dairy Sci 91(3):1218-1228.

Humblot, C. and J. P. Guyot. 2009. Pyrosequencing of tagged 16S rRNA gene amplicons for rapid deciphering of the microbiomes of fermented foods such as pearl millet slurries. Applied and Environmental Microbiology 75(13):4354.

Jayarao, B. and D. Wolfgang. 2003. Bulk-tank milk analysis: a useful tool for improving milk quality and herd udder health. The Veterinary clinics of North America. Food animal practice 19(1).

Jayarao, B. M. and L. Wang. 1999. A study on the prevalence of gram-negative bacteria in bulk tank milk. J Dairy Sci 82(12):2620-2624 
Kaplan, C. W., J. C. Astaire, M. E. Sanders, B. S. Reddy, and C. L. Kitts. 2001. 16S ribosomal DNA terminal restriction fragment pattern analysis of bacterial communities in feces of rats fed Lactobacillus acidophilus NCFM. Appl Environ Microb 67(4):1935-1939.

Kent, A., D. Smith, B. Benson, and E. Triplett. 2003. Web-based phylogenetic assignment tool for analysis of terminal restriction fragment length polymorphism profiles of microbial communities. Appl Environ Microb 69(11):6768.

Kitts, C. 2001. Terminal restriction fragment patterns: a tool for comparing microbial communities and assessing community dynamics. Current issues in intestinal microbiology 2(1):17-25.

Knaysi, G. and M. Ford. 1938. A Method of Counting Viable Bacteria in Milk by Means of the Microscope1. Journal of Dairy Science 21(3):129-141.

Kurweil, R. and M. Busse. 1973. Total count and microflora of freshly drawn milk. Milchwissenschaft 28:427.

Lafarge, V., J. Ogier, V. Girard, V. Maladen, J. Leveau, A. Gruss, and A. Delacroix-Buchet. 2004. Raw cow milk bacterial population shifts attributable to refrigeration. Appl Environ Microb 70(9):5644.

Liu, W., T. Marsh, H. Cheng, and L. Forney. 1997. Characterization of microbial diversity by determining terminal restriction fragment length polymorphisms of genes encoding 16S rRNA. Appl Environ Microb 63(11):4516.

Lockley, A. and R. Bardsley. 2000. DNA-based methods for food authentication. Trends in Food Science \& Technology 11(2):67-77.

Lord, N., C. Kaplan, P. Shank, C. Kitts, and S. Elrod. 2002. Assessment of fungal diversity using terminal restriction fragment (TRF) pattern analysis: comparison of $18 \mathrm{~S}$ and ITS ribosomal regions. Fems Microbiol Ecol 42(3):327-337.

Marsh, T. L. 1999. Terminal restriction fragment length polymorphism (T-RFLP): an emerging method for characterizing diversity among homologous populations of amplification products. Curr Opin Microbiol 2(3):323-327.

Marsh, T. L., P. Saxman, J. Cole, and J. Tiedje. 2000. Terminal restriction fragment length polymorphism analysis program, a web-based research tool for microbial community analysis. Appl Environ Microb 66(8):3616-3620.

Marth, E. and J. Steele. 2001. Applied dairy microbiology. CRC.

Martin, J. 1974. Significance of bacterial spores in milk. J Milk Food Technol 37:794.

Martins, M. L., C. L. Pinto, R. B. Rocha, E. F. de Araujo, and M. C. Vanetti. 2006. Genetic diversity of Gram-negative, proteolytic, psychrotrophic bacteria isolated from refrigerated raw milk. Int J Food Microbiol 111(2):144-148.

McKenzie, J. J., Goldman, R. 1999. The student edition of Minitab for Windows user's manual: release 12. Addison-Wesley:464p.

Murillo, C. 2003. Dominant spores and microflora in milk powder production using terminal restriction fragment analysis and conventional methods. Thesis submitted to the Department of Agriculture, Cal Poly, San Luis Obispo, CA.

Murphy, M. A., M. R. Shariflou, and C. Moran. 2002. High quality genomic DNA extraction from large milk samples. Journal of Dairy Research 69(04):645-649.

Murphy, S. and K. Boor. 2000. Sources and causes of high bacteria counts in raw milk: an abbreviated review. Dairy, Food and Environmental Sanitation 20(8):1-4. 
Muyzer, G. and K. Smalla. 1998. Application of denaturing gradient gel electrophoresis (DGGE) and temperature gradient gel electrophoresis (TGGE) in microbial ecology. Antonie Van Leeuwenhoek 73(1):127-141.

Nagashima, K., T. Hisada, M. Sato, and J. Mochizuki. 2003. Application of new primer-enzyme combinations to terminal restriction fragment length polymorphism profiling of bacterial populations in human feces. Appl Environ Microb 69(2):1251-1262.

Nicodème, M., J. Grill, G. Humbert, and J. Gaillard. 2005. Extracellular protease activity of different Pseudomonas strains: dependence of proteolytic activity on culture conditions. Journal of Applied Microbiology 99(3):641-648.

Nielsen, S. S. 2002. Plasmin system and microbial proteases in milk: characteristics, roles, and relationship. J Agric Food Chem 50(22):6628-6634.

Nornberg, M. F. B. L., R. S. C. Friedrich, R. D. N. Weiss, E. C. Tondo, and A. Brandelli. 2010. Proteolytic activity among psychrotrophic bacteria isolated from refrigerated raw milk. International Journal of Dairy Technology 63(1):41-46.

Nunan, N., T. Daniell, B. Singh, A. Papert, J. McNicol, and J. Prosser. 2005. Links between plant and rhizoplane bacterial communities in grassland soils, characterized using molecular techniques. Appl Environ Microb 71(11):6784.

Osborn, A. M., E. R. B. Moore, and K. N. Timmis. 2000. An evaluation of terminal-restriction fragment length polymorphism (T-RFLP) analysis for the study of microbial community structure and dynamics. Environ Microbiol 2(1):39-50.

Osborne, C. A., G. N. Rees, Y. Bernstein, and P. H. Janssen. 2006. New threshold and confidence estimates for terminal restriction fragment length polymorphism analysis of complex bacterial communities. Appl Environ Microb 72(2):1270-1278.

Pantoja, J. C., D. J. Reinemann, and P. L. Ruegg. 2009. Associations among milk quality indicators in raw bulk milk. J Dairy Sci 92(10):4978-4987.

Park, Y. K., H. C. Koo, S. H. Kim, S. Y. Hwang, W. K. Jung, J. M. Kim, S. Shin, R. T. Kim, and Y. H. Park. 2007. The analysis of milk components and pathogenic bacteria isolated from bovine raw milk in Korea. J Dairy Sci 90(12):5405-5414.

Phillips, J. and M. Griffiths. 1986. Factors contributing to the seasonal variation of Bacillus spp. in pasteurized dairy products. Journal of Applied Microbiology 61(4):275-285.

Pitkala, A., M. Haveri, S. Pyorala, V. Myllys, and T. Honkanen-Buzalski. 2004a. Bovine mastitis in Finland 2001--prevalence, distribution of bacteria, and antimicrobial resistance. J Dairy Sci 87(8):2433-2441.

Pitkala, A., M. Haveri, S. Pyorala, V. Myllys, and T. Honkanen-Buzalski. 2004b. Bovine mastitis in Finland 2001 - Prevalence, distribution of bacteria, and antimicrobial resistance. Journal of Dairy Science 87(8):2433-2441.

R, Development, Core, and Team. 2010. R: A language and environment for statistical computing. R Foundation for Statistical Computing.

Rademaker, J., M. Peinhopf, L. Rijnen, W. Bockelmann, and W. Noordman. 2005. The surface microflora dynamics of bacterial smear-ripened Tilsit cheese determined by T-RFLP DNA population fingerprint analysis. Int Dairy J 15(6-9):785-794.

Ramesh, A., B. Padmapriya, A. Chrashekar, and M. Varadaraj. 2002. Application of a convenient DNA extraction method and multiplex PCR for the direct detection of Staphylococcus aureus and Yersinia enterocolitica in milk samples. Molecular and cellular probes 16(4):307-314. 
Rees, G., D. Baldwin, G. Watson, S. Perryman, and D. Nielsen. 2004. Ordination and significance testing of microbial community composition derived from terminal restriction fragment length polymorphisms: application of multivariate statistics. Antonie Van Leeuwenhoek 86(4):339-347.

Reinemann, D., G. Wolters, P. Billon, O. Lind, and M. Rasmussen. 2003. Review of practices for cleaning and sanitation of milking machines. Bulletin-International Dairy Federation:3-18. Roose-Amsaleg, C., E. Garnier-Sillam, and M. Harry. 2001. Extraction and purification of microbial DNA from soil and sediment samples. Applied Soil Ecology 18(1):47-60.

Rowan, N. J. 2004. Viable but non-culturable forms of food and waterborne bacteria: Quo Vadis? Trends in Food Science \& Technology 15(9):462-467.

Rueckert, A., R. Ronimus, and H. Morgan. 2005. Development of a rapid detection and enumeration method for thermophilic bacilli in milk powders. J Microbiol Meth 60(2):155-167. Saboyainsta, L. V. and J. L. Maubois. 2000. Current developments of microfiltration technology in the dairy industry. Le Lait 80(6):541-553.

Sait, L., M. Galic, R. Strugnell, and P. Janssen. 2003. Secretory antibodies do not affect the composition of the bacterial microbiota in the terminal ileum of 10-week-old mice. Appl Environ Microb 69(4):2100.

Sakai, M., A. Matsuka, T. Komura, and S. Kanazawa. 2004. Application of a new PCR primer for terminal restriction fragment length polymorphism analysis of the bacterial communities in plant roots. J Microbiol Methods 59(1):81-89.

Sambrook, J., E. Fritsch, and T. Maniatis. 1989. Molecular cloning. Cold Spring Harbor Laboratory Press Cold Spring Harbor, NY.

Scheldeman, P., L. Herman, S. Foster, and M. Heyndrickx. 2006. Bacillus sporothermodurans and other highly heat-resistant spore formers in milk. Journal of Applied Microbiology 101(3):542-555.

Schukken, Y., D. Wilson, F. Welcome, L. Garrison-Tikofsky, and R. Gonzalez. 2003. Monitoring udder health and milk quality using somatic cell counts. Veterinary research 34(5):579-596.

Schutte, U. M. E., Z. Abdo, S. J. Bent, C. Shyu, C. J. Williams, J. D. Pierson, and L. J. Forney. 2008. Advances in the use of terminal restriction fragment length polymorphism (T-RFLP) analysis of $16 \mathrm{~S}$ rRNA genes to characterize microbial communities. Appl Microbiol Biot 80(3):365-380.

Shendure, J. and H. Ji. 2008. Next-generation DNA sequencing. Nature biotechnology 26(10):1135-1145.

Smalla, K., M. Oros-Sichler, A. Milling, H. Heuer, S. Baumgarte, R. Becker, G. Neuber, S. Kropf, A. Ulrich, and C. Tebbe. 2007. Bacterial diversity of soils assessed by DGGE, T-RFLP and SSCP fingerprints of PCR-amplified 16S rRNA gene fragments: do the different methods provide similar results? J Microbiol Meth 69(3):470-479.

Smit, E., P. Leeflang, and K. Wernars. 1997. Detection of shifts in microbial community structure and diversity in soil caused by copper contamination using amplified ribosomal DNA restriction analysis. Fems Microbiol Ecol 23(3):249-261.

Sørhaug, T. and L. Stepaniak. 1997. Psychrotrophs and their enzymes in milk and dairy products: quality aspects. Trends in Food Science \& Technology 8(2):35-41.

Sutherland, A. and R. Murdoch. 1994. Seasonal occurrence of psychrotrophic Bacillus species in raw milk, and studies on the interactions with mesophilic Bacillus sp. International Journal of Food Microbiology 21(4):279-292. 
Tanaka, Y., H. Takahashi, N. Kitazawa, and B. Kimura. 2010. Rapid estimation of microbial populations in fish samples by using terminal restriction fragment length polymorphism analysis of 16S rDNA. Journal of Food Protection\&\# 174; 73(1):104-113.

Taponen, S., L. Salmikivi, H. Simojoki, M. T. Koskinen, and S. Pyorala. 2009. Real-time polymerase chain reaction-based identification of bacteria in milk samples from bovine clinical mastitis with no growth in conventional culturing. Journal of Dairy Science 92(6):2610-2617. Tolle, A. 1980. The microflora of the udder. Factors influencing the bacteriological quality of raw milk. International Dairy Federation Bulletin, Document 120(4).

Veal, D., D. Deere, B. Ferrari, J. Piper, and P. Attfield. 2000. Fluorescence staining and flow cytometry for monitoring microbial cells. Journal of immunological methods 243(1-2):191-210. Wagner, A., L. Adrian, S. Kleinsteuber, J. R. Andreesen, and U. Lechner. 2009. Transcription analysis of genes encoding homologues of reductive dehalogenases in "Dehalococcoides" sp. strain CBDB1 by using terminal restriction fragment length polymorphism and quantitative PCR. Appl Environ Microbiol 75(7):1876-1884.

Wegmuller, B., J. Luthy, and U. Candrian. 1993. Direct polymerase chain reaction detection of Campylobacter jejuni and Campylobacter coli in raw milk and dairy products. Appl Environ Microb 59(7):2161.

Wehr, H. and J. Frank. 2004. Standard methods for the examination of Dairy products. Amer Public Health Assn.

Whitfield, F., N. JENSEN, and K. SHAW. 2001. Role of Yersinia intermedia and Pseudomonas putida in the development of a fruity off-flavour in pasteurized milk. Journal of Dairy Research 67(04):561-569.

Widjojoatmodjo, M., A. Fluit, and J. Verhoef. 1994. Rapid identification of bacteria by PCRsingle-strand conformation polymorphism. J Clin Microbiol 32(12):3002.

Wiedmann, M., D. Weilmeier, S. Dineen, R. Ralyea, and K. Boor. 2000. Molecular and phenotypic characterization of Pseudomonas spp. isolated from milk. Applied and Environmental Microbiology 66(5):2085.

Wintzingerode, F., U. Göbel, and E. Stackebrandt. 1997. Determination of microbial diversity in environmental samples: pitfalls of PCR-based rRNA analysis. FEMS microbiology reviews 21(3):213-229. 


\section{Appendices}

Appendix 1. Principal Component Analysis: pH, SPC, SCC, CC, PSC and LPC

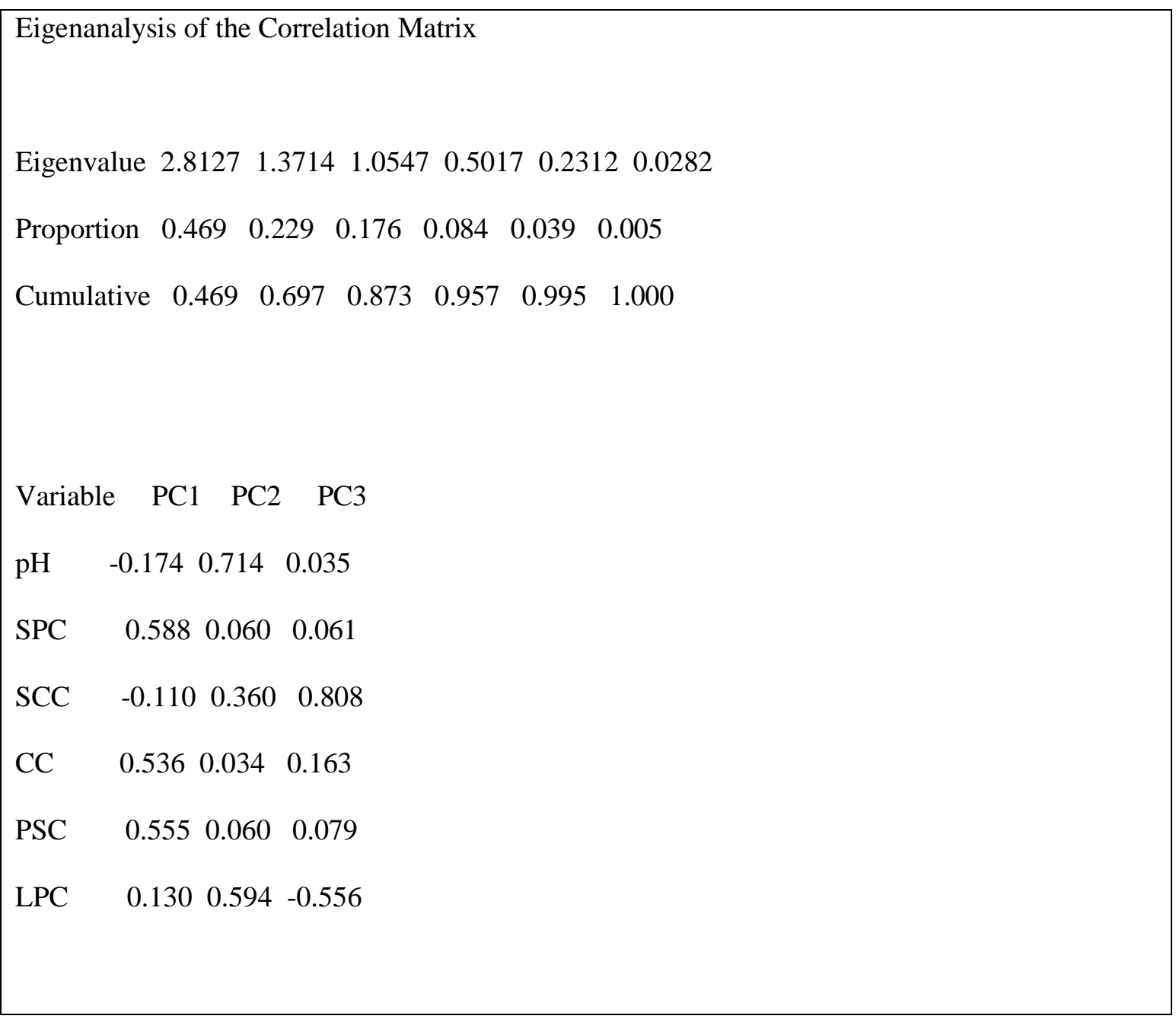


Appendix 2. Grouping of raw milk quality

\begin{tabular}{|c|c|c|c|c|}
\hline Conventional tests & SPC & SCC & $\mathrm{CC}$ & LPC \\
\hline \multirow[t]{23}{*}{ High quality } & $1-243$ & $1-167$ & $1-268$ & $1-167$ \\
\hline & $1-268$ & $1-201$ & $3-183$ & $1-201$ \\
\hline & $3-183$ & $1-240$ & $3-193$ & $1-240$ \\
\hline & $3-193$ & $1-243$ & $3-201$ & $1-243$ \\
\hline & $3-201$ & $1-268$ & $3-243$ & $1-268$ \\
\hline & $3-225$ & $12-157$ & $3-807$ & $1-807$ \\
\hline & $3-243$ & $12-213$ & $3-844$ & $1-844$ \\
\hline & $3-807$ & $12-278$ & $5-111$ & $5-179$ \\
\hline & $5-111$ & $3-201$ & $5-179$ & $5-190$ \\
\hline & $5-179$ & $3-225$ & $5-190$ & $5-205$ \\
\hline & $5-190$ & $3-240$ & $5-205$ & $5-209$ \\
\hline & $5-205$ & $3-243$ & $5-209$ & $5-259$ \\
\hline & $5-209$ & $5-190$ & $7-111$ & $5-703$ \\
\hline & $5-259$ & $5-205$ & $7-190$ & $7-190$ \\
\hline & $5-703$ & $5-209$ & $7-205$ & $7-205$ \\
\hline & $7-111$ & $7-179$ & $7-209$ & $7-209$ \\
\hline & $7-179$ & $7-190$ & $7-212$ & $7-212$ \\
\hline & $7-190$ & $7-205$ & $7-255$ & $7-844$ \\
\hline & $7-205$ & $7-209$ & $7-844$ & \\
\hline & $7-209$ & & & \\
\hline & $7-212$ & & & \\
\hline & $7-255$ & & & \\
\hline & $7-844$ & & & \\
\hline \multirow[t]{18}{*}{ Low quality } & $1-167$ & $1-807$ & $1-167$ & $12-154$ \\
\hline & $1-201$ & $1-844$ & $1-201$ & $12-157$ \\
\hline & $1-240$ & $12-154$ & $1-240$ & $12-158$ \\
\hline & $1-807$ & $12-158$ & $1-243$ & $12-213$ \\
\hline & $1-844$ & $12-703$ & $1-807$ & $12-278$ \\
\hline & $12-154$ & $3-183$ & $1-844$ & $12-703$ \\
\hline & $12-157$ & $3-193$ & $12-154$ & $3-183$ \\
\hline & $12-158$ & $3-807$ & $12-157$ & $3-193$ \\
\hline & $12-213$ & $3-844$ & $12-158$ & $3-201$ \\
\hline & $12-278$ & $5-111$ & $12-213$ & $3-225$ \\
\hline & $12-703$ & $5-179$ & $12-278$ & $3-240$ \\
\hline & $3-240$ & $5-259$ & $12-703$ & $3-243$ \\
\hline & $3-844$ & $5-703$ & $3-225$ & $3-807$ \\
\hline & & $7-111$ & $3-240$ & $3-844$ \\
\hline & & $7-212$ & $5-259$ & $5-111$ \\
\hline & & $7-255$ & $5-703$ & $7-111$ \\
\hline & & $7-844$ & $7-179$ & $7-179$ \\
\hline & & & & $7-255$ \\
\hline
\end{tabular}


Appendix 3. Assumption tests of data of conventional microbial tests for MANOVA
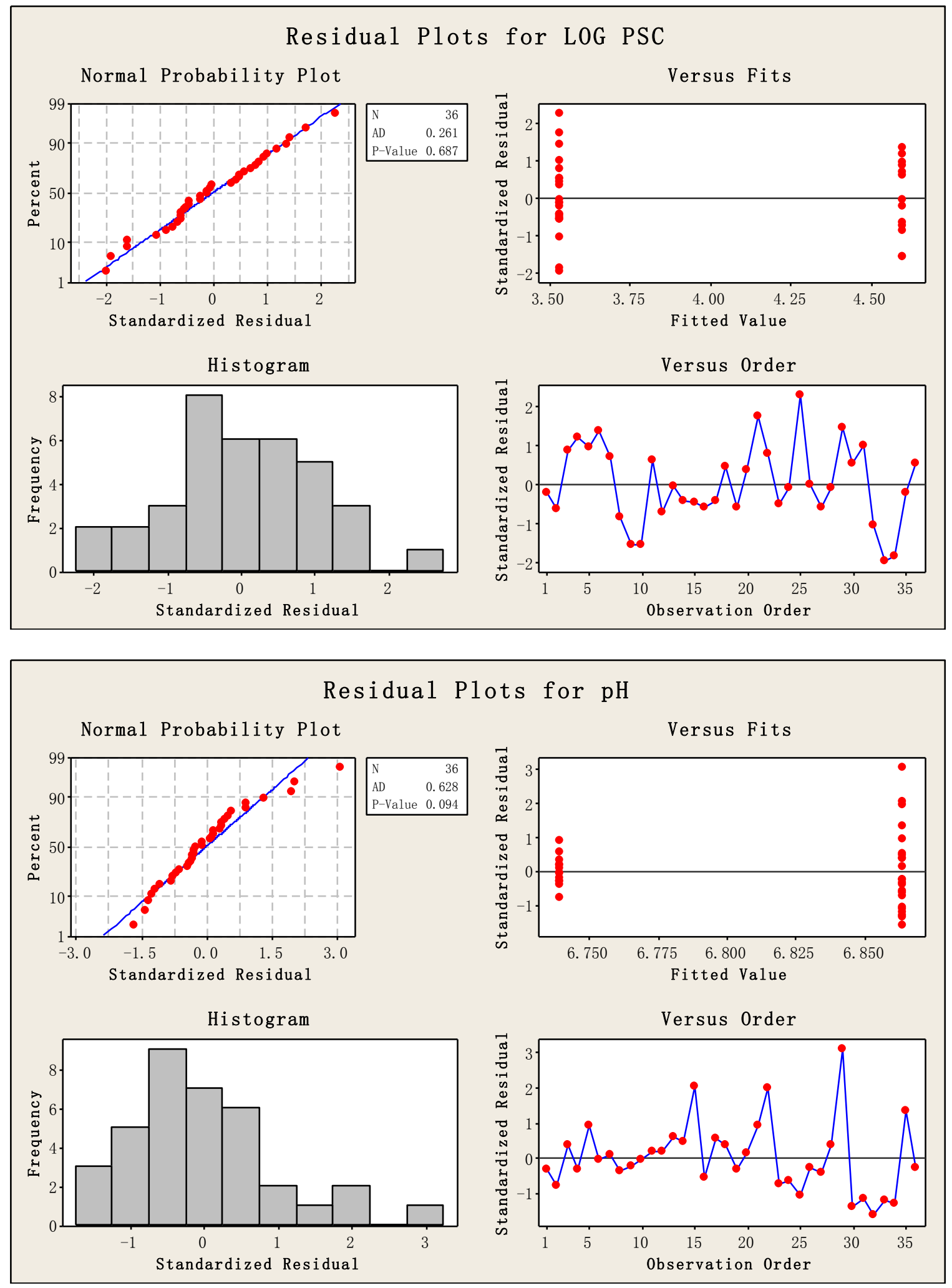

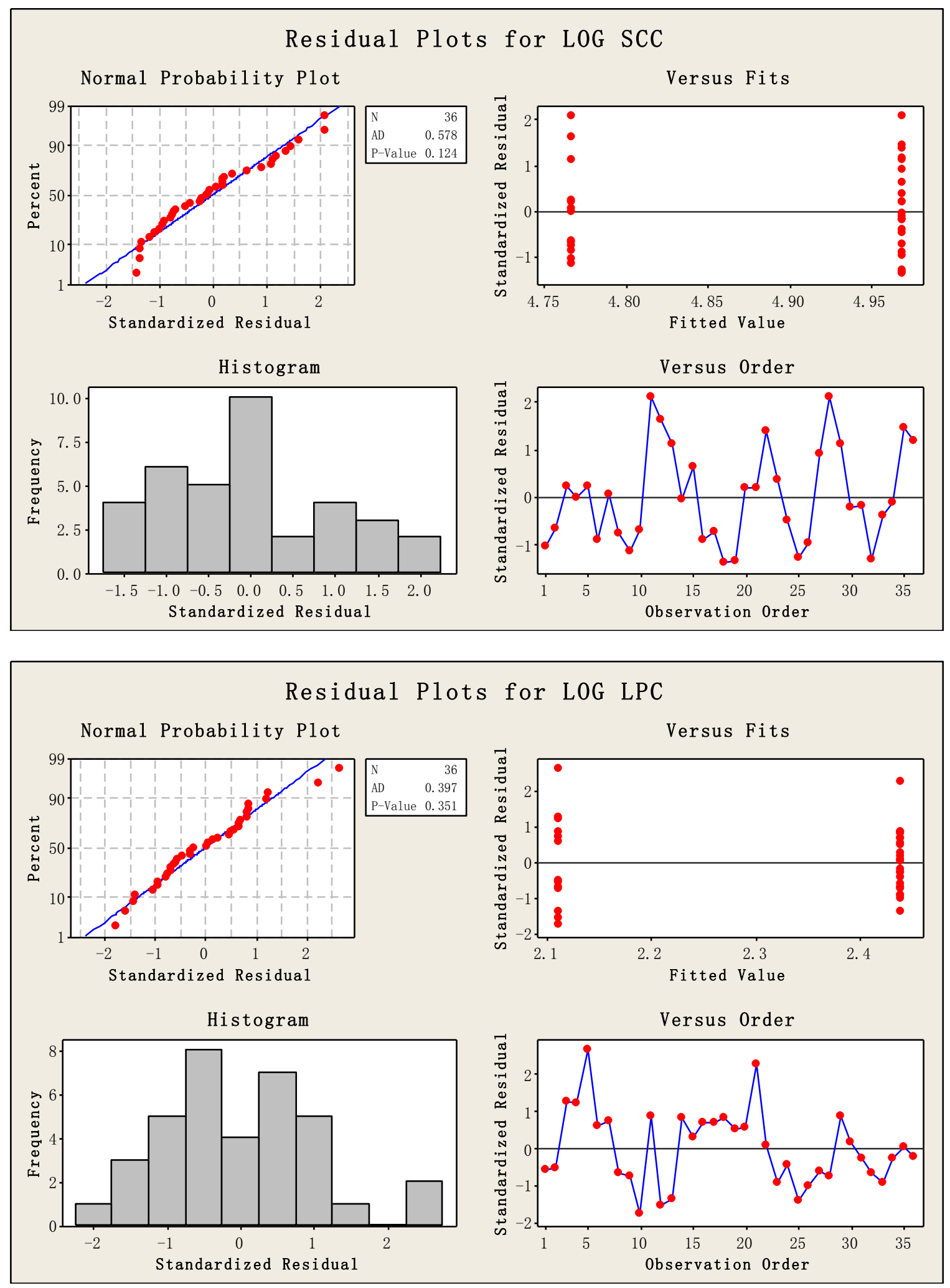

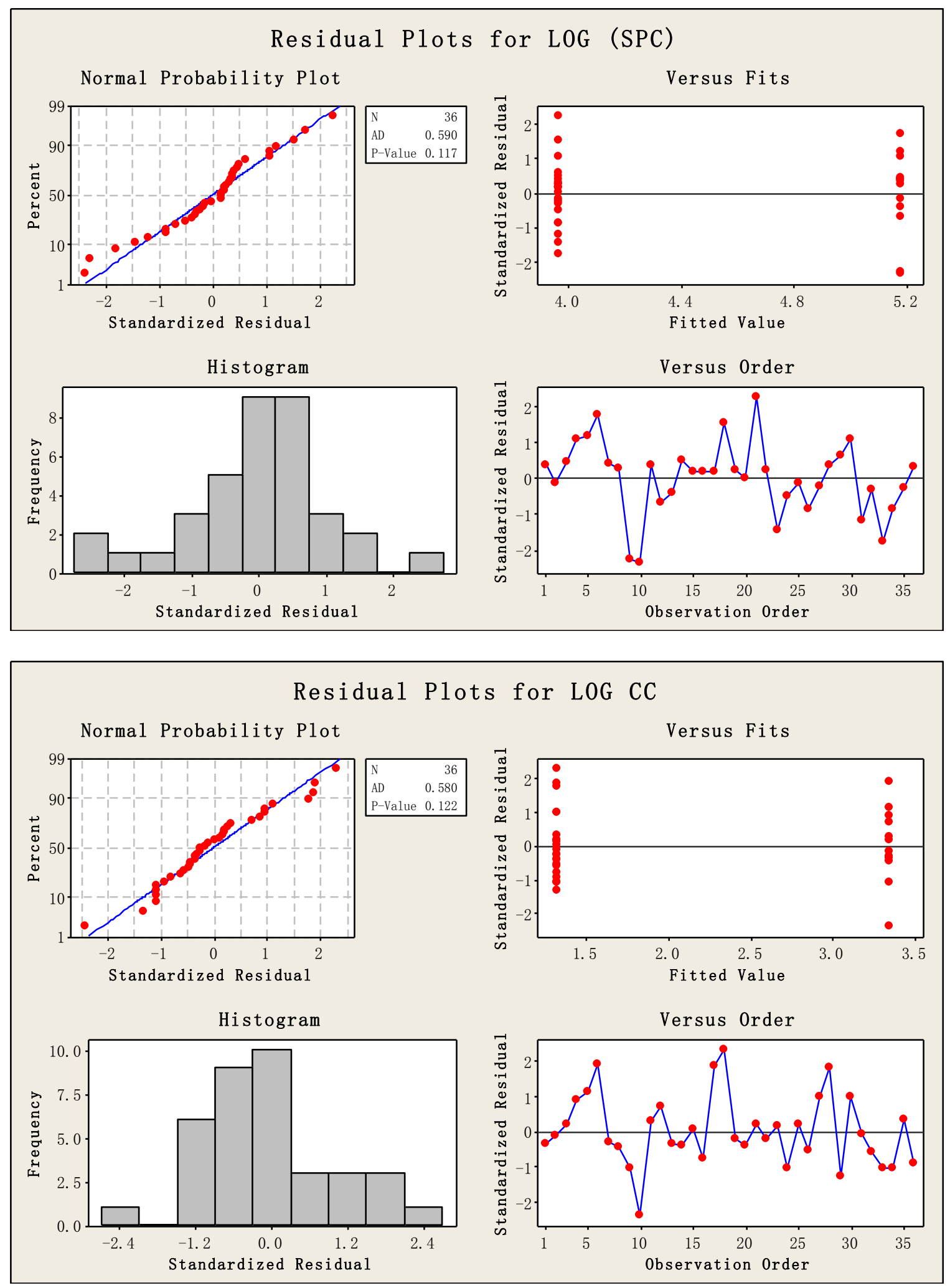
Appendix 4. DpnII Macro TRF results used for analysis in peak aera. Noise peaks have been truncated.

\begin{tabular}{|c|c|c|c|c|c|c|c|c|c|c|c|c|c|c|c|c|c|c|}
\hline $\mathrm{BP}$ & $12-158$ & $12-154$ & $12-157$ & $12-703$ & $12-213$ & $12-278$ & $7-255$ & $7-212$ & $7-209$ & $7-205$ & $7-190$ & $7-111$ & $7-844$ & $7-179$ & $5-111$ & $5-179$ & $5-190$ & $5-703$ \\
\hline 56 & 0 & 0 & 0 & 0 & 0 & 167263 & 37066 & 0 & 0 & 0 & 0 & 0 & 22103 & 0 & 68091 & 18492 & 22027 & 0 \\
\hline 57 & 0 & 0 & 0 & 0 & 0 & 125749 & 0 & 0 & 0 & 0 & 0 & 0 & 0 & 0 & 0 & 0 & 0 & 24582 \\
\hline 58 & 0 & 0 & 0 & 0 & 0 & 0 & 0 & 0 & 0 & 0 & 0 & 0 & 0 & 0 & 37766 & 0 & 0 & 0 \\
\hline 59 & 0 & 0 & 0 & 0 & 0 & 0 & 0 & 0 & 0 & 0 & 0 & 0 & 0 & 0 & 33199 & 0 & 0 & 0 \\
\hline 61 & 0 & 53900 & 13137 & 0 & 0 & 0 & 16925 & 0 & 13645 & 0 & 0 & 0 & 20818 & 25226 & 47973 & 0 & 24355 & 0 \\
\hline 62 & 0 & 14683 & 0 & 0 & 0 & 0 & 0 & 0 & 0 & 0 & 0 & 0 & 0 & 0 & 0 & 0 & 0 & 0 \\
\hline 63 & 0 & 0 & 0 & 0 & 0 & 224181 & 0 & 0 & 0 & 0 & 23392 & 0 & 75126 & 45064 & 97650 & 32852 & 0 & 0 \\
\hline 64 & 0 & 0 & 0 & 0 & 0 & 0 & 0 & 0 & 0 & 0 & 0 & 0 & 0 & 0 & 0 & 0 & 0 & 0 \\
\hline 66 & 0 & 0 & 0 & 0 & 15849 & 0 & 0 & 0 & 0 & 0 & 0 & 0 & 0 & 0 & 49867 & 0 & 0 & 0 \\
\hline 67 & 0 & 0 & 0 & 0 & 0 & 0 & 15345 & 0 & 0 & 0 & 0 & 19971 & 0 & 0 & 0 & 0 & 0 & 0 \\
\hline 68 & 0 & 0 & 0 & 0 & 13605 & 39473 & 0 & 0 & 0 & 0 & 0 & 0 & 0 & 0 & 47594 & 16491 & 0 & 0 \\
\hline 69 & 0 & 0 & 0 & 0 & 0 & 0 & 0 & 0 & 0 & 0 & 0 & 0 & 0 & 0 & 34800 & 0 & 0 & 0 \\
\hline 70 & 0 & 0 & 0 & 0 & 0 & 0 & 0 & 0 & 0 & 0 & 0 & 0 & 0 & 0 & 0 & 0 & 0 & 0 \\
\hline 71 & 0 & 0 & 0 & 0 & 0 & 0 & 19219 & 0 & 0 & 0 & 0 & 0 & 21801 & 0 & 58669 & 28786 & 0 & 0 \\
\hline 73 & 0 & 0 & 0 & 0 & 0 & 56274 & 0 & 0 & 0 & 0 & 0 & 0 & 0 & 0 & 27319 & 0 & 0 & 0 \\
\hline 74 & 0 & 18244 & 19250 & 0 & 0 & 0 & 0 & 0 & 0 & 0 & 0 & 0 & 0 & 0 & 23653 & 22655 & 0 & 0 \\
\hline 75 & 0 & 40072 & 26501 & 0 & 0 & 36566 & 19033 & 0 & 0 & 0 & 0 & 0 & 31534 & 17544 & 43660 & 53668 & 32772 & 0 \\
\hline 76 & 0 & 0 & 0 & 12652 & 0 & 0 & 0 & 0 & 0 & 0 & 0 & 0 & 0 & 0 & 0 & 0 & 0 & 0 \\
\hline 77 & 0 & 0 & 0 & 257016 & 0 & 0 & 0 & 0 & 0 & 0 & 0 & 0 & 30216 & 0 & 48855 & 32949 & 0 & 0 \\
\hline 78 & 0 & 0 & 0 & 55657 & 0 & 33353 & 0 & 0 & 0 & 0 & 0 & 0 & 0 & 0 & 0 & 19750 & 31340 & 0 \\
\hline 79 & 0 & 12533 & 10603 & 0 & 0 & 0 & 0 & 0 & 14772 & 0 & 27241 & 22486 & 20393 & 0 & 45365 & 28270 & 0 & 0 \\
\hline 81 & 0 & 59125 & 55748 & 88768 & 0 & 52434 & 19461 & 14938 & 19198 & 21302 & 58108 & 0 & 33166 & 0 & 42033 & 31239 & 23102 & 31794 \\
\hline 82 & 0 & 0 & 0 & 0 & 0 & 0 & 0 & 0 & 0 & 0 & 0 & 16084 & 0 & 0 & 25763 & 0 & 0 & 0 \\
\hline 83 & 0 & 0 & 0 & 117774 & 0 & 17029 & 0 & 0 & 0 & 0 & 0 & 0 & 0 & 0 & 0 & 0 & 119628 & 0 \\
\hline 84 & 0 & 0 & 0 & 0 & 0 & 0 & 0 & 16006 & 0 & 0 & 0 & 22410 & 0 & 0 & 0 & 0 & 0 & 0 \\
\hline 85 & 0 & 0 & 0 & 0 & 0 & 0 & 0 & 0 & 0 & 0 & 0 & 39485 & 0 & 0 & 23784 & 0 & 0 & 0 \\
\hline 86 & 0 & 0 & 0 & 0 & 0 & 0 & 21030 & 0 & 0 & 0 & 0 & 0 & 0 & 0 & 0 & 0 & 0 & 0 \\
\hline 87 & 0 & 0 & 12651 & 0 & 29531 & 0 & 0 & 0 & 0 & 0 & 0 & 0 & 0 & 0 & 27789 & 0 & 80229 & 0 \\
\hline 88 & 0 & 0 & 0 & 0 & 0 & 0 & 0 & 0 & 0 & 0 & 0 & 0 & 0 & 0 & 0 & 0 & 0 & 0 \\
\hline
\end{tabular}




\begin{tabular}{|c|c|c|c|c|c|c|c|c|c|c|c|c|c|c|c|c|c|c|}
\hline 89 & 0 & 22221 & 0 & 0 & 0 & 0 & 0 & 0 & 0 & 0 & 0 & 0 & 0 & 0 & 25182 & 0 & 0 & 0 \\
\hline 92 & 0 & 0 & 0 & 17498 & 0 & 0 & 0 & 0 & 0 & 0 & 0 & 0 & 0 & 0 & 0 & 0 & 0 & 0 \\
\hline 93 & 0 & 0 & 0 & 0 & 0 & 0 & 0 & 0 & 0 & 0 & 0 & 0 & 0 & 0 & 0 & 0 & 0 & 0 \\
\hline 94 & 0 & 0 & 0 & 0 & 0 & 0 & 0 & 0 & 0 & 0 & 0 & 0 & 0 & 0 & 0 & 0 & 0 & 0 \\
\hline 95 & 0 & 0 & 0 & 42129 & 0 & 0 & 0 & 0 & 0 & 0 & 0 & 0 & 0 & 0 & 0 & 0 & 0 & 0 \\
\hline 98 & 0 & 0 & 0 & 12585 & 0 & 0 & 0 & 0 & 16018 & 0 & 0 & 19895 & 20371 & 21786 & 34937 & 23720 & 18983 & 0 \\
\hline 99 & 0 & 0 & 0 & 0 & 0 & 0 & 0 & 0 & 0 & 0 & 14208 & 48860 & 26986 & 32565 & 0 & 26108 & 0 & 0 \\
\hline 101 & 0 & 0 & 0 & 15142 & 0 & 0 & 23401 & 0 & 0 & 0 & 54616 & 45278 & 46027 & 68914 & 42726 & 38371 & 0 & 0 \\
\hline 102 & 0 & 0 & 0 & 0 & 0 & 0 & 0 & 0 & 0 & 0 & 0 & 0 & 0 & 0 & 0 & 0 & 0 & 0 \\
\hline 103 & 0 & 0 & 0 & 0 & 0 & 0 & 0 & 0 & 0 & 0 & 21876 & 0 & 0 & 0 & 0 & 0 & 0 & 0 \\
\hline 104 & 0 & 91428 & 118246 & 13662 & 0 & 22248 & 23862 & 0 & 0 & 0 & 43262 & 21267 & 0 & 0 & 26129 & 24559 & 19699 & 52454 \\
\hline 105 & 0 & 0 & 0 & 0 & 0 & 0 & 0 & 0 & 0 & 0 & 0 & 13339 & 0 & 0 & 0 & 0 & 0 & 0 \\
\hline 106 & 0 & 0 & 0 & 0 & 0 & 0 & 0 & 0 & 0 & 0 & 26929 & 0 & 0 & 0 & 0 & 15974 & 0 & 0 \\
\hline 107 & 0 & 0 & 0 & 0 & 0 & 0 & 0 & 0 & 35031 & 0 & 0 & 0 & 0 & 0 & 0 & 0 & 0 & 0 \\
\hline 108 & 0 & 0 & 0 & 0 & 0 & 0 & 0 & 0 & 0 & 0 & 0 & 0 & 0 & 0 & 0 & 0 & 0 & 0 \\
\hline 109 & 0 & 0 & 0 & 0 & 0 & 0 & 0 & 0 & 0 & 0 & 15605 & 0 & 0 & 0 & 0 & 15845 & 0 & 0 \\
\hline 114 & 0 & 0 & 11438 & 0 & 0 & 0 & 0 & 0 & 0 & 0 & 0 & 0 & 0 & 0 & 0 & 0 & 0 & 0 \\
\hline 115 & 0 & 0 & 0 & 0 & 0 & 0 & 0 & 0 & 44583 & 25765 & 0 & 21496 & 0 & 20411 & 0 & 0 & 0 & 0 \\
\hline 116 & 0 & 0 & 0 & 0 & 0 & 0 & 32753 & 19441 & 56916 & 70527 & 37808 & 0 & 0 & 41050 & 0 & 0 & 0 & 0 \\
\hline 117 & 0 & 0 & 0 & 0 & 0 & 0 & 0 & 0 & 0 & 0 & 0 & 0 & 0 & 0 & 0 & 0 & 0 & 0 \\
\hline 118 & 0 & 0 & 0 & 0 & 0 & 0 & 0 & 0 & 0 & 29830 & 0 & 0 & 0 & 0 & 0 & 0 & 0 & 0 \\
\hline 119 & 0 & 0 & 0 & 0 & 0 & 0 & 0 & 0 & 13726 & 72520 & 20345 & 0 & 0 & 59282 & 0 & 0 & 0 & 0 \\
\hline 120 & 0 & 0 & 0 & 0 & 0 & 0 & 0 & 0 & 0 & 32480 & 34048 & 0 & 0 & 0 & 0 & 0 & 0 & 0 \\
\hline 121 & 0 & 0 & 0 & 0 & 0 & 0 & 0 & 0 & 30861 & 0 & 0 & 0 & 0 & 0 & 0 & 0 & 0 & 0 \\
\hline 122 & 0 & 0 & 0 & 0 & 0 & 0 & 0 & 0 & 0 & 0 & 0 & 0 & 0 & 0 & 0 & 0 & 0 & 0 \\
\hline 124 & 0 & 0 & 0 & 0 & 0 & 0 & 0 & 0 & 0 & 0 & 25770 & 17761 & 0 & 0 & 0 & 0 & 0 & 0 \\
\hline 126 & 0 & 0 & 0 & 0 & 0 & 0 & 0 & 0 & 0 & 0 & 21921 & 27136 & 0 & 0 & 0 & 21848 & 0 & 16792 \\
\hline 127 & 0 & 0 & 0 & 0 & 0 & 0 & 0 & 0 & 0 & 0 & 0 & 0 & 0 & 0 & 0 & 0 & 0 & 0 \\
\hline 128 & 0 & 0 & 0 & 0 & 0 & 0 & 0 & 0 & 13460 & 0 & 47869 & 0 & 32126 & 37037 & 0 & 36564 & 0 & 24345 \\
\hline 129 & 0 & 0 & 0 & 0 & 0 & 0 & 0 & 0 & 0 & 0 & 0 & 0 & 0 & 0 & 0 & 0 & 0 & 0 \\
\hline 130 & 0 & 0 & 0 & 0 & 0 & 0 & 0 & 0 & 0 & 0 & 0 & 0 & 0 & 0 & 0 & 0 & 0 & 0 \\
\hline 132 & 0 & 0 & 0 & 0 & 0 & 0 & 0 & 0 & 0 & 0 & 0 & 0 & 0 & 0 & 0 & 0 & 0 & 0 \\
\hline
\end{tabular}




\begin{tabular}{|c|c|c|c|c|c|c|c|c|c|c|c|c|c|c|c|c|c|c|}
\hline 134 & 0 & 0 & 0 & 0 & 0 & 0 & 0 & 0 & 0 & 0 & 0 & 0 & 0 & 0 & 0 & 0 & 0 & 0 \\
\hline 135 & 0 & 0 & 0 & 0 & 0 & 0 & 0 & 0 & 0 & 0 & 0 & 0 & 0 & 0 & 0 & 0 & 0 & 0 \\
\hline 137 & 0 & 0 & 0 & 0 & 0 & 0 & 0 & 0 & 0 & 0 & 21267 & 0 & 0 & 0 & 0 & 0 & 0 & 0 \\
\hline 138 & 0 & 0 & 0 & 45965 & 0 & 0 & 0 & 0 & 0 & 0 & 0 & 0 & 0 & 0 & 0 & 0 & 256268 & 0 \\
\hline 139 & 0 & 0 & 0 & 0 & 0 & 0 & 0 & 0 & 0 & 17956 & 0 & 0 & 0 & 0 & 0 & 0 & 0 & 0 \\
\hline 142 & 0 & 0 & 0 & 0 & 0 & 0 & 0 & 0 & 0 & 0 & 0 & 0 & 0 & 0 & 0 & 0 & 0 & 0 \\
\hline 146 & 0 & 0 & 0 & 27795 & 0 & 0 & 0 & 0 & 0 & 0 & 0 & 0 & 0 & 0 & 0 & 32304 & 23460 & 24582 \\
\hline 147 & 0 & 0 & 0 & 0 & 0 & 0 & 0 & 0 & 0 & 0 & 14074 & 0 & 0 & 0 & 0 & 0 & 0 & 17081 \\
\hline 149 & 0 & 0 & 0 & 0 & 0 & 0 & 0 & 0 & 0 & 0 & 0 & 21114 & 0 & 0 & 0 & 0 & 0 & 0 \\
\hline 150 & 0 & 0 & 0 & 0 & 0 & 0 & 15246 & 0 & 0 & 16236 & 34434 & 0 & 29914 & 0 & 0 & 30787 & 0 & 31741 \\
\hline 151 & 0 & 0 & 0 & 0 & 0 & 0 & 0 & 0 & 0 & 0 & 0 & 0 & 0 & 0 & 0 & 0 & 0 & 0 \\
\hline 152 & 0 & 0 & 0 & 0 & 0 & 0 & 0 & 0 & 0 & 0 & 0 & 0 & 23477 & 0 & 0 & 16426 & 0 & 0 \\
\hline 153 & 0 & 0 & 0 & 0 & 0 & 0 & 0 & 0 & 0 & 0 & 0 & 0 & 0 & 0 & 0 & 0 & 0 & 0 \\
\hline 156 & 0 & 0 & 0 & 0 & 0 & 0 & 0 & 0 & 0 & 0 & 0 & 0 & 0 & 0 & 0 & 20783 & 0 & 20029 \\
\hline 157 & 0 & 0 & 0 & 12248 & 0 & 0 & 0 & 0 & 0 & 0 & 0 & 0 & 0 & 0 & 0 & 0 & 0 & 0 \\
\hline 158 & 0 & 0 & 0 & 0 & 0 & 0 & 0 & 0 & 0 & 0 & 0 & 0 & 0 & 0 & 0 & 0 & 0 & 0 \\
\hline 160 & 0 & 0 & 0 & 0 & 0 & 0 & 0 & 0 & 0 & 0 & 13673 & 0 & 0 & 0 & 0 & 0 & 0 & 0 \\
\hline 161 & 0 & 0 & 0 & 0 & 0 & 0 & 0 & 0 & 0 & 0 & 0 & 0 & 0 & 0 & 0 & 0 & 0 & 19924 \\
\hline 162 & 0 & 0 & 0 & 0 & 0 & 0 & 0 & 0 & 0 & 0 & 0 & 0 & 0 & 0 & 0 & 0 & 0 & 0 \\
\hline 165 & 0 & 0 & 0 & 0 & 0 & 0 & 0 & 0 & 0 & 0 & 0 & 0 & 25455 & 0 & 27475 & 0 & 0 & 0 \\
\hline 167 & 27018 & 0 & 0 & 0 & 59698 & 0 & 0 & 0 & 0 & 0 & 0 & 0 & 0 & 0 & 0 & 0 & 0 & 0 \\
\hline 170 & 0 & 0 & 0 & 0 & 0 & 0 & 0 & 0 & 0 & 0 & 0 & 12044 & 24684 & 0 & 0 & 0 & 0 & 0 \\
\hline 171 & 0 & 0 & 0 & 0 & 0 & 0 & 0 & 0 & 0 & 29017 & 0 & 0 & 0 & 0 & 0 & 0 & 0 & 0 \\
\hline 172 & 0 & 0 & 0 & 0 & 0 & 0 & 0 & 0 & 0 & 0 & 0 & 0 & 0 & 0 & 0 & 16620 & 0 & 0 \\
\hline 175 & 0 & 0 & 0 & 0 & 0 & 0 & 0 & 0 & 0 & 0 & 0 & 0 & 0 & 0 & 0 & 0 & 0 & 0 \\
\hline 176 & 0 & 0 & 0 & 0 & 0 & 0 & 0 & 0 & 0 & 0 & 16600 & 0 & 0 & 0 & 0 & 0 & 0 & 0 \\
\hline 177 & 0 & 0 & 0 & 0 & 0 & 0 & 0 & 0 & 0 & 0 & 29247 & 0 & 20885 & 0 & 0 & 24139 & 0 & 21871 \\
\hline 178 & 0 & 0 & 0 & 0 & 0 & 0 & 0 & 0 & 48500 & 0 & 28935 & 0 & 22025 & 0 & 0 & 17653 & 0 & 23319 \\
\hline 179 & 0 & 0 & 0 & 0 & 0 & 0 & 0 & 0 & 0 & 0 & 32131 & 0 & 21913 & 0 & 0 & 20073 & 0 & 24187 \\
\hline 180 & 0 & 0 & 0 & 0 & 0 & 0 & 0 & 13801 & 0 & 0 & 15560 & 0 & 0 & 0 & 0 & 18492 & 0 & 22924 \\
\hline 182 & 0 & 0 & 0 & 0 & 0 & 0 & 0 & 0 & 0 & 0 & 0 & 0 & 0 & 0 & 0 & 0 & 0 & 0 \\
\hline 184 & 0 & 0 & 0 & 0 & 0 & 0 & 0 & 0 & 0 & 17370 & 0 & 0 & 0 & 0 & 0 & 0 & 0 & 0 \\
\hline
\end{tabular}




\begin{tabular}{|c|c|c|c|c|c|c|c|c|c|c|c|c|c|c|c|c|c|c|}
\hline 185 & 0 & 0 & 0 & 0 & 0 & 0 & 0 & 42504 & 0 & 0 & 0 & 0 & 0 & 0 & 0 & 0 & 0 & 0 \\
\hline 187 & 0 & 0 & 0 & 0 & 0 & 0 & 0 & 0 & 0 & 0 & 0 & 0 & 0 & 0 & 0 & 0 & 0 & 33899 \\
\hline 188 & 0 & 0 & 0 & 0 & 0 & 0 & 0 & 452749 & 0 & 25515 & 0 & 0 & 0 & 122807 & 0 & 0 & 21848 & 301671 \\
\hline 189 & 0 & 0 & 0 & 0 & 0 & 0 & 0 & 0 & 0 & 0 & 0 & 0 & 0 & 0 & 0 & 0 & 0 & 0 \\
\hline 190 & 0 & 0 & 0 & 0 & 0 & 0 & 0 & 0 & 0 & 24210 & 0 & 0 & 0 & 24653 & 0 & 0 & 23997 & 0 \\
\hline 191 & 0 & 0 & 0 & 0 & 0 & 0 & 0 & 0 & 0 & 0 & 0 & 0 & 41815 & 0 & 0 & 16555 & 0 & 0 \\
\hline 192 & 0 & 0 & 0 & 0 & 0 & 0 & 0 & 0 & 0 & 0 & 0 & 0 & 0 & 0 & 0 & 0 & 0 & 0 \\
\hline 193 & 0 & 0 & 0 & 0 & 0 & 0 & 0 & 0 & 0 & 0 & 0 & 11815 & 0 & 0 & 0 & 0 & 0 & 0 \\
\hline 194 & 0 & 0 & 0 & 0 & 0 & 0 & 0 & 0 & 0 & 0 & 0 & 0 & 0 & 0 & 28377 & 0 & 0 & 0 \\
\hline 197 & 0 & 0 & 0 & 0 & 0 & 0 & 0 & 0 & 0 & 0 & 0 & 0 & 26841 & 0 & 0 & 15264 & 0 & 0 \\
\hline 199 & 0 & 0 & 0 & 0 & 0 & 0 & 0 & 0 & 0 & 0 & 0 & 25840 & 0 & 0 & 0 & 0 & 0 & 0 \\
\hline 200 & 0 & 0 & 0 & 0 & 0 & 0 & 17265 & 0 & 0 & 0 & 0 & 0 & 34328 & 0 & 0 & 29174 & 0 & 16739 \\
\hline 201 & 0 & 12833 & 18127 & 0 & 0 & 0 & 0 & 0 & 0 & 0 & 0 & 36512 & 66678 & 22130 & 0 & 34208 & 0 & 0 \\
\hline 202 & 0 & 79011 & 35618 & 25035 & 11980 & 39655 & 30514 & 0 & 22636 & 31081 & 210052 & 117921 & 103632 & 58480 & 31344 & 65834 & 105838 & 32004 \\
\hline 203 & 0 & 0 & 0 & 0 & 0 & 0 & 0 & 0 & 0 & 0 & 0 & 19056 & 25165 & 0 & 0 & 22881 & 0 & 16581 \\
\hline 204 & 0 & 0 & 0 & 0 & 0 & 0 & 0 & 0 & 0 & 0 & 0 & 0 & 0 & 0 & 0 & 0 & 0 & 0 \\
\hline 205 & 0 & 0 & 0 & 0 & 0 & 0 & 0 & 0 & 0 & 0 & 0 & 0 & 0 & 0 & 0 & 0 & 0 & 0 \\
\hline 206 & 0 & 0 & 0 & 0 & 0 & 0 & 0 & 0 & 0 & 0 & 0 & 0 & 0 & 0 & 0 & 0 & 0 & 0 \\
\hline 210 & 0 & 0 & 0 & 0 & 0 & 0 & 0 & 0 & 0 & 0 & 0 & 0 & 0 & 0 & 0 & 0 & 0 & 0 \\
\hline 211 & 0 & 0 & 0 & 0 & 0 & 0 & 21195 & 0 & 0 & 0 & 16942 & 0 & 28137 & 0 & 0 & 21106 & 0 & 0 \\
\hline 212 & 0 & 0 & 0 & 0 & 0 & 0 & 0 & 0 & 0 & 0 & 0 & 0 & 31177 & 0 & 0 & 16717 & 0 & 0 \\
\hline 216 & 0 & 0 & 0 & 0 & 0 & 0 & 0 & 23724 & 0 & 0 & 0 & 0 & 0 & 0 & 0 & 0 & 0 & 0 \\
\hline 217 & 0 & 0 & 0 & 0 & 0 & 0 & 0 & 0 & 0 & 0 & 0 & 0 & 0 & 0 & 0 & 0 & 0 & 0 \\
\hline 219 & 0 & 0 & 0 & 0 & 0 & 0 & 0 & 0 & 0 & 0 & 18532 & 0 & 27411 & 0 & 0 & 0 & 0 & 0 \\
\hline 221 & 0 & 0 & 0 & 0 & 0 & 0 & 0 & 0 & 0 & 0 & 0 & 0 & 0 & 0 & 0 & 0 & 0 & 0 \\
\hline 222 & 0 & 0 & 0 & 0 & 0 & 0 & 0 & 0 & 0 & 0 & 0 & 0 & 0 & 0 & 0 & 0 & 0 & 0 \\
\hline 224 & 0 & 0 & 0 & 0 & 0 & 0 & 0 & 0 & 0 & 0 & 0 & 0 & 0 & 0 & 0 & 0 & 0 & 0 \\
\hline 225 & 0 & 0 & 0 & 0 & 0 & 0 & 0 & 0 & 0 & 0 & 0 & 0 & 0 & 0 & 0 & 0 & 0 & 0 \\
\hline 226 & 0 & 17065 & 0 & 0 & 0 & 0 & 0 & 0 & 0 & 0 & 0 & 0 & 27377 & 0 & 0 & 0 & 0 & 0 \\
\hline 227 & 0 & 0 & 0 & 0 & 0 & 0 & 0 & 0 & 0 & 0 & 0 & 0 & 0 & 0 & 0 & 22655 & 0 & 0 \\
\hline 228 & 0 & 0 & 0 & 0 & 12460 & 0 & 0 & 0 & 0 & 0 & 0 & 0 & 0 & 0 & 0 & 0 & 0 & 0 \\
\hline 229 & 0 & 0 & 0 & 0 & 0 & 0 & 15981 & 0 & 27776 & 20387 & 0 & 0 & 0 & 0 & 0 & 0 & 0 & 0 \\
\hline
\end{tabular}




\begin{tabular}{|c|c|c|c|c|c|c|c|c|c|c|c|c|c|c|c|c|c|c|}
\hline 230 & 0 & 0 & 0 & 0 & 0 & 0 & 0 & 0 & 0 & 0 & 0 & 0 & 0 & 0 & 0 & 0 & 0 & 0 \\
\hline 231 & 0 & 0 & 0 & 0 & 0 & 0 & 0 & 0 & 0 & 0 & 0 & 0 & 0 & 0 & 0 & 0 & 0 & 0 \\
\hline 232 & 0 & 0 & 0 & 0 & 0 & 0 & 0 & 0 & 0 & 0 & 0 & 0 & 0 & 0 & 0 & 0 & 0 & 0 \\
\hline 233 & 0 & 0 & 0 & 0 & 0 & 0 & 0 & 0 & 0 & 0 & 0 & 0 & 0 & 0 & 0 & 0 & 0 & 0 \\
\hline 234 & 0 & 0 & 0 & 0 & 0 & 0 & 0 & 0 & 0 & 0 & 0 & 0 & 0 & 0 & 0 & 0 & 0 & 0 \\
\hline 235 & 0 & 0 & 0 & 0 & 0 & 0 & 0 & 0 & 0 & 0 & 0 & 0 & 0 & 0 & 0 & 0 & 0 & 0 \\
\hline 236 & 0 & 0 & 0 & 0 & 0 & 0 & 0 & 0 & 0 & 0 & 0 & 0 & 0 & 0 & 0 & 0 & 0 & 0 \\
\hline 238 & 0 & 0 & 0 & 0 & 0 & 0 & 0 & 17967 & 0 & 24444 & 0 & 0 & 0 & 0 & 0 & 0 & 18983 & 0 \\
\hline 239 & 0 & 0 & 0 & 0 & 0 & 0 & 0 & 0 & 0 & 0 & 0 & 0 & 0 & 0 & 0 & 0 & 0 & 0 \\
\hline 240 & 0 & 0 & 0 & 0 & 0 & 0 & 0 & 0 & 0 & 0 & 0 & 0 & 0 & 23965 & 0 & 0 & 0 & 0 \\
\hline 241 & 0 & 0 & 0 & 0 & 0 & 0 & 0 & 0 & 0 & 14493 & 0 & 0 & 0 & 0 & 0 & 0 & 14506 & 0 \\
\hline 242 & 26594 & 0 & 14669 & 11239 & 13404 & 17001 & 0 & 154859 & 16997 & 0 & 0 & 0 & 0 & 86114 & 0 & 0 & 14864 & 68746 \\
\hline 243 & 0 & 0 & 0 & 0 & 0 & 0 & 0 & 0 & 0 & 51320 & 0 & 13111 & 0 & 31304 & 0 & 0 & 0 & 0 \\
\hline 244 & 0 & 0 & 0 & 0 & 0 & 0 & 0 & 0 & 0 & 22076 & 0 & 0 & 0 & 0 & 0 & 0 & 0 & 0 \\
\hline 250 & 136234 & 0 & 0 & 0 & 0 & 0 & 0 & 0 & 0 & 0 & 0 & 0 & 0 & 0 & 0 & 0 & 0 & 0 \\
\hline 253 & 0 & 0 & 0 & 0 & 0 & 0 & 0 & 0 & 0 & 0 & 0 & 0 & 0 & 0 & 0 & 0 & 0 & 0 \\
\hline 254 & 0 & 0 & 0 & 0 & 0 & 0 & 0 & 0 & 0 & 0 & 0 & 0 & 0 & 0 & 0 & 0 & 0 & 0 \\
\hline 255 & 0 & 0 & 26319 & 0 & 0 & 0 & 0 & 0 & 0 & 0 & 0 & 0 & 0 & 0 & 0 & 0 & 0 & 0 \\
\hline 256 & 0 & 0 & 47920 & 0 & 0 & 0 & 0 & 0 & 0 & 0 & 0 & 0 & 0 & 0 & 0 & 0 & 0 & 0 \\
\hline 257 & 0 & 0 & 0 & 0 & 0 & 0 & 0 & 0 & 0 & 0 & 0 & 0 & 0 & 0 & 0 & 0 & 0 & 0 \\
\hline 258 & 0 & 0 & 0 & 0 & 0 & 0 & 0 & 0 & 0 & 0 & 0 & 0 & 0 & 0 & 0 & 0 & 0 & 0 \\
\hline 260 & 0 & 0 & 0 & 0 & 0 & 0 & 0 & 0 & 0 & 17284 & 0 & 0 & 0 & 0 & 0 & 0 & 0 & 0 \\
\hline 261 & 0 & 0 & 0 & 0 & 0 & 0 & 0 & 0 & 20135 & 0 & 0 & 0 & 0 & 0 & 0 & 0 & 0 & 0 \\
\hline 262 & 0 & 0 & 0 & 0 & 0 & 0 & 0 & 0 & 0 & 0 & 0 & 0 & 0 & 0 & 0 & 0 & 0 & 0 \\
\hline 264 & 0 & 0 & 0 & 0 & 0 & 0 & 0 & 0 & 0 & 0 & 0 & 0 & 0 & 0 & 0 & 0 & 0 & 0 \\
\hline 265 & 0 & 0 & 0 & 0 & 0 & 0 & 0 & 0 & 18290 & 0 & 0 & 0 & 0 & 0 & 0 & 0 & 0 & 0 \\
\hline 266 & 46274 & 0 & 13395 & 0 & 34918 & 0 & 0 & 19825 & 39410 & 0 & 0 & 17227 & 0 & 22016 & 0 & 0 & 0 & 15949 \\
\hline 268 & 582432 & 294425 & 379206 & 33448 & 315126 & 79793 & 28790 & 27949 & 375055 & 42690 & 0 & 0 & 0 & 0 & 0 & 0 & 0 & 0 \\
\hline 270 & 88264 & 102227 & 119930 & 0 & 226160 & 29211 & 36561 & 0 & 0 & 0 & 0 & 0 & 0 & 0 & 0 & 0 & 0 & 18055 \\
\hline 272 & 23710 & 0 & 29808 & 0 & 28092 & 0 & 77524 & 0 & 0 & 40446 & 0 & 0 & 0 & 0 & 0 & 0 & 0 & 0 \\
\hline 273 & 0 & 0 & 0 & 184198 & 0 & 45952 & 0 & 0 & 0 & 0 & 0 & 0 & 0 & 0 & 0 & 0 & 0 & 0 \\
\hline 274 & 17390 & 0 & 23740 & 27189 & 38060 & 0 & 0 & 0 & 0 & 0 & 0 & 0 & 0 & 86573 & 0 & 0 & 0 & 0 \\
\hline
\end{tabular}




\begin{tabular}{|c|c|c|c|c|c|c|c|c|c|c|c|c|c|c|c|c|c|c|}
\hline 275 & 0 & 0 & 0 & 0 & 0 & 0 & 18231 & 0 & 0 & 0 & 0 & 0 & 0 & 0 & 0 & 0 & 0 & 0 \\
\hline 276 & 0 & 0 & 10679 & 0 & 0 & 0 & 0 & 0 & 0 & 0 & 0 & 0 & 0 & 0 & 0 & 15039 & 0 & 0 \\
\hline 277 & 0 & 0 & 0 & 0 & 0 & 0 & 0 & 0 & 21195 & 55862 & 28014 & 41467 & 0 & 37037 & 0 & 0 & 0 & 0 \\
\hline 278 & 0 & 0 & 0 & 0 & 0 & 0 & 34487 & 0 & 0 & 0 & 0 & 0 & 0 & 0 & 0 & 0 & 0 & 0 \\
\hline 280 & 0 & 0 & 0 & 0 & 0 & 0 & 25717 & 0 & 0 & 0 & 0 & 0 & 0 & 0 & 0 & 0 & 0 & 0 \\
\hline 282 & 0 & 0 & 0 & 0 & 0 & 0 & 38098 & 0 & 0 & 0 & 0 & 0 & 0 & 0 & 0 & 0 & 0 & 0 \\
\hline 283 & 0 & 0 & 0 & 0 & 0 & 0 & 0 & 0 & 0 & 0 & 0 & 0 & 0 & 0 & 0 & 0 & 0 & 0 \\
\hline 284 & 0 & 0 & 0 & 0 & 0 & 0 & 0 & 0 & 0 & 0 & 0 & 0 & 0 & 0 & 0 & 0 & 0 & 0 \\
\hline 285 & 0 & 0 & 0 & 0 & 0 & 0 & 29657 & 0 & 0 & 0 & 0 & 0 & 0 & 0 & 0 & 0 & 0 & 0 \\
\hline 286 & 0 & 0 & 0 & 0 & 0 & 0 & 137531 & 16389 & 34750 & 55760 & 0 & 0 & 0 & 41968 & 0 & 0 & 0 & 0 \\
\hline 289 & 0 & 0 & 0 & 0 & 0 & 0 & 64035 & 0 & 13103 & 0 & 0 & 0 & 0 & 0 & 0 & 0 & 0 & 0 \\
\hline 290 & 0 & 0 & 0 & 0 & 0 & 0 & 78797 & 0 & 0 & 50452 & 0 & 0 & 0 & 21213 & 0 & 0 & 0 & 0 \\
\hline 291 & 0 & 0 & 0 & 0 & 0 & 0 & 0 & 0 & 0 & 0 & 0 & 0 & 0 & 0 & 0 & 0 & 0 & 0 \\
\hline 292 & 10985 & 0 & 0 & 0 & 19363 & 0 & 30964 & 12326 & 38321 & 43675 & 0 & 17151 & 0 & 31074 & 0 & 0 & 0 & 0 \\
\hline 293 & 0 & 98319 & 0 & 0 & 0 & 0 & 0 & 0 & 0 & 0 & 0 & 0 & 0 & 0 & 0 & 0 & 0 & 0 \\
\hline 294 & 0 & 0 & 0 & 0 & 0 & 0 & 0 & 0 & 0 & 0 & 0 & 0 & 0 & 0 & 0 & 0 & 0 & 0 \\
\hline 295 & 0 & 0 & 0 & 0 & 0 & 0 & 0 & 0 & 0 & 0 & 0 & 0 & 0 & 0 & 0 & 0 & 0 & 0 \\
\hline 298 & 0 & 0 & 0 & 0 & 0 & 0 & 14236 & 0 & 0 & 30589 & 0 & 12196 & 0 & 21786 & 0 & 0 & 0 & 0 \\
\hline 301 & 0 & 0 & 0 & 0 & 0 & 0 & 0 & 0 & 0 & 0 & 0 & 0 & 0 & 0 & 0 & 0 & 0 & 0 \\
\hline 302 & 0 & 0 & 0 & 0 & 15013 & 0 & 0 & 0 & 0 & 0 & 0 & 0 & 0 & 0 & 0 & 0 & 0 & 20055 \\
\hline 303 & 0 & 0 & 0 & 0 & 32519 & 0 & 0 & 0 & 0 & 89265 & 14178 & 85830 & 0 & 0 & 0 & 0 & 0 & 0 \\
\hline 304 & 0 & 0 & 0 & 0 & 0 & 0 & 0 & 0 & 0 & 0 & 0 & 0 & 0 & 0 & 0 & 0 & 0 & 0 \\
\hline 305 & 14421 & 24395 & 0 & 0 & 83951 & 0 & 57076 & 0 & 22218 & 0 & 33364 & 0 & 0 & 0 & 0 & 0 & 0 & 0 \\
\hline 306 & 0 & 0 & 0 & 0 & 0 & 0 & 0 & 0 & 25218 & 0 & 0 & 0 & 0 & 0 & 0 & 0 & 0 & 0 \\
\hline 307 & 0 & 0 & 0 & 0 & 31590 & 0 & 0 & 0 & 0 & 0 & 0 & 0 & 0 & 0 & 0 & 0 & 0 & 0 \\
\hline 310 & 0 & 0 & 0 & 0 & 0 & 0 & 0 & 0 & 0 & 0 & 0 & 0 & 0 & 0 & 0 & 0 & 0 & 0 \\
\hline 311 & 0 & 0 & 0 & 0 & 0 & 0 & 0 & 0 & 0 & 0 & 0 & 0 & 0 & 0 & 0 & 0 & 0 & 0 \\
\hline 312 & 0 & 0 & 0 & 0 & 0 & 0 & 0 & 0 & 0 & 27446 & 0 & 43677 & 0 & 0 & 0 & 0 & 0 & 0 \\
\hline 313 & 0 & 0 & 0 & 0 & 0 & 0 & 0 & 0 & 0 & 0 & 0 & 0 & 0 & 0 & 0 & 0 & 0 & 0 \\
\hline 315 & 26679 & 0 & 13015 & 0 & 18682 & 0 & 0 & 0 & 0 & 0 & 0 & 0 & 0 & 0 & 0 & 0 & 0 & 0 \\
\hline 317 & 0 & 0 & 0 & 0 & 0 & 0 & 0 & 0 & 0 & 0 & 0 & 0 & 0 & 0 & 0 & 0 & 0 & 0 \\
\hline 318 & 0 & 0 & 0 & 0 & 0 & 0 & 0 & 0 & 0 & 0 & 0 & 0 & 0 & 0 & 0 & 0 & 0 & 0 \\
\hline
\end{tabular}




\begin{tabular}{|c|c|c|c|c|c|c|c|c|c|c|c|c|c|c|c|c|c|c|}
\hline 320 & 0 & 0 & 0 & 0 & 0 & 0 & 0 & 0 & 0 & 0 & 0 & 0 & 0 & 0 & 0 & 0 & 0 & 0 \\
\hline 322 & 0 & 0 & 0 & 0 & 0 & 0 & 0 & 0 & 0 & 0 & 0 & 0 & 0 & 0 & 0 & 0 & 0 & 0 \\
\hline 329 & 0 & 0 & 0 & 0 & 0 & 0 & 0 & 0 & 0 & 0 & 0 & 0 & 0 & 0 & 0 & 0 & 0 & 0 \\
\hline 332 & 0 & 0 & 0 & 0 & 0 & 0 & 0 & 0 & 0 & 0 & 0 & 0 & 38418 & 0 & 0 & 0 & 0 & 0 \\
\hline 358 & 0 & 0 & 0 & 0 & 0 & 0 & 0 & 0 & 0 & 0 & 0 & 0 & 0 & 0 & 0 & 0 & 0 & 0 \\
\hline 359 & 0 & 0 & 0 & 0 & 0 & 0 & 0 & 0 & 0 & 0 & 0 & 0 & 0 & 0 & 0 & 0 & 0 & 0 \\
\hline 362 & 0 & 0 & 0 & 0 & 0 & 0 & 0 & 0 & 0 & 0 & 0 & 0 & 0 & 0 & 0 & 0 & 0 & 0 \\
\hline 386 & 0 & 0 & 0 & 0 & 0 & 0 & 0 & 0 & 0 & 0 & 0 & 0 & 0 & 0 & 0 & 0 & 0 & 0 \\
\hline 402 & 0 & 0 & 0 & 0 & 0 & 0 & 0 & 0 & 0 & 0 & 0 & 0 & 0 & 0 & 0 & 0 & 0 & 0 \\
\hline 410 & 0 & 0 & 0 & 0 & 0 & 0 & 0 & 0 & 0 & 0 & 0 & 0 & 0 & 0 & 0 & 0 & 0 & 0 \\
\hline 430 & 0 & 59519 & 0 & 0 & 0 & 13816 & 0 & 0 & 0 & 0 & 0 & 0 & 0 & 0 & 0 & 55152 & 99212 & 42611 \\
\hline 439 & 0 & 0 & 0 & 0 & 0 & 0 & 0 & 0 & 0 & 0 & 0 & 0 & 0 & 0 & 0 & 0 & 0 & 0 \\
\hline 451 & 0 & 0 & 0 & 0 & 0 & 0 & 0 & 0 & 0 & 0 & 0 & 0 & 0 & 0 & 0 & 0 & 0 & 0 \\
\hline 459 & 0 & 0 & 0 & 0 & 0 & 0 & 0 & 0 & 0 & 0 & 0 & 0 & 0 & 0 & 0 & 0 & 0 & 0 \\
\hline 476 & 0 & 0 & 0 & 0 & 0 & 0 & 0 & 0 & 0 & 0 & 0 & 0 & 0 & 0 & 0 & 0 & 0 & 0 \\
\hline 502 & 0 & 0 & 0 & 0 & 0 & 0 & 0 & 0 & 0 & 0 & 0 & 0 & 0 & 0 & 0 & 0 & 0 & 0 \\
\hline 503 & 0 & 0 & 0 & 0 & 0 & 0 & 0 & 18722 & 0 & 0 & 0 & 0 & 0 & 0 & 0 & 0 & 0 & 0 \\
\hline 504 & 0 & 0 & 0 & 0 & 0 & 0 & 0 & 0 & 0 & 0 & 0 & 0 & 0 & 0 & 0 & 0 & 0 & 0 \\
\hline 506 & 0 & 0 & 0 & 0 & 0 & 0 & 0 & 25222 & 0 & 0 & 0 & 0 & 0 & 0 & 0 & 0 & 0 & 0 \\
\hline 507 & 0 & 0 & 0 & 0 & 0 & 0 & 0 & 30561 & 18186 & 0 & 0 & 0 & 0 & 0 & 0 & 0 & 28832 & 41900 \\
\hline 508 & 0 & 0 & 0 & 0 & 0 & 0 & 0 & 25280 & 0 & 0 & 0 & 0 & 0 & 0 & 0 & 0 & 0 & 0 \\
\hline 509 & 0 & 0 & 0 & 0 & 0 & 0 & 0 & 20161 & 0 & 0 & 0 & 0 & 0 & 0 & 0 & 0 & 0 & 0 \\
\hline 511 & 0 & 0 & 0 & 0 & 0 & 0 & 0 & 22192 & 0 & 0 & 0 & 0 & 0 & 0 & 0 & 0 & 20057 & 36163 \\
\hline 512 & 0 & 0 & 0 & 0 & 0 & 0 & 0 & 0 & 0 & 0 & 0 & 189572 & 0 & 0 & 0 & 0 & 0 & 0 \\
\hline 513 & 0 & 0 & 0 & 0 & 0 & 0 & 0 & 25384 & 0 & 0 & 0 & 0 & 0 & 0 & 0 & 0 & 0 & 0 \\
\hline 515 & 0 & 0 & 0 & 0 & 0 & 0 & 0 & 0 & 0 & 0 & 0 & 0 & 0 & 0 & 0 & 0 & 0 & 0 \\
\hline 518 & 0 & 0 & 0 & 0 & 0 & 0 & 0 & 0 & 0 & 0 & 0 & 0 & 0 & 0 & 0 & 0 & 0 & 0 \\
\hline 526 & 0 & 0 & 0 & 0 & 0 & 0 & 0 & 0 & 0 & 0 & 0 & 0 & 0 & 0 & 0 & 0 & 0 & 0 \\
\hline 528 & 0 & 0 & 0 & 0 & 0 & 0 & 0 & 0 & 0 & 0 & 0 & 0 & 0 & 0 & 0 & 0 & 0 & 0 \\
\hline 530 & 0 & 0 & 0 & 0 & 0 & 0 & 0 & 0 & 0 & 0 & 0 & 0 & 0 & 0 & 0 & 0 & 0 & 0 \\
\hline
\end{tabular}




\begin{tabular}{|c|c|c|c|c|c|c|c|c|c|c|c|c|c|c|c|c|c|c|}
\hline $\mathrm{BP}$ & $5-205$ & $5-259$ & 5-209 & $3-225$ & $3-243$ & $3-807$ & $3-844$ & 3-193 & $3-201$ & $3-240$ & 3-183 & $1-807$ & $1-844$ & $1-201$ & $1-240$ & $1-167$ & $1-268$ & $1-243$ \\
\hline 56 & 0 & 0 & 0 & 0 & 44201 & 0 & 0 & 0 & 76541 & 0 & 0 & 0 & 0 & 0 & 0 & 96955 & 0 & 0 \\
\hline 57 & 0 & 0 & 0 & 0 & 0 & 0 & 0 & 0 & 52137 & 0 & 0 & 0 & 0 & 0 & 0 & 82670 & 0 & 0 \\
\hline 58 & 0 & 0 & 0 & 0 & 0 & 0 & 0 & 0 & 0 & 0 & 0 & 0 & 0 & 0 & 0 & 0 & 0 & $\begin{array}{c}0 \\
35059\end{array}$ \\
\hline 59 & 0 & 0 & 0 & 0 & 29516 & 27750 & 0 & 0 & 50786 & 0 & 0 & 0 & 24512 & 0 & 0 & 58970 & 0 & 1 \\
\hline 61 & 0 & 0 & 11905 & 26621 & 31897 & 43441 & 0 & 0 & 42008 & 0 & 50123 & 0 & 41407 & 0 & 0 & 0 & 0 & 0 \\
\hline 62 & 0 & 0 & 0 & 0 & 0 & 0 & 0 & 0 & 0 & 0 & 36185 & 0 & 32267 & 0 & 26027 & $\begin{array}{c}0 \\
14601\end{array}$ & 0 & 0 \\
\hline 63 & 0 & 0 & 0 & 55821 & 46691 & 32233 & 0 & 0 & 63278 & 20783 & 55336 & 0 & 0 & 0 & 0 & 4 & 0 & $\begin{array}{c}0 \\
15290\end{array}$ \\
\hline 64 & 0 & 0 & 0 & 0 & 0 & 0 & 0 & 0 & 0 & 0 & 15365 & 0 & 0 & 0 & 0 & 0 & 0 & $\begin{array}{c}5 \\
17481\end{array}$ \\
\hline 66 & 0 & 0 & 0 & 0 & 0 & 0 & 0 & 0 & 0 & 0 & 0 & 90780 & 0 & 0 & 0 & 30468 & 0 & 3 \\
\hline 67 & 0 & 0 & 0 & 0 & 0 & 0 & 0 & 0 & 0 & 0 & 0 & 0 & 0 & 0 & 0 & 0 & 0 & 0 \\
\hline 68 & 67827 & 0 & 0 & 0 & 22522 & 0 & 0 & 0 & 0 & 0 & 0 & 0 & 0 & 0 & 0 & 16558 & 0 & 18454 \\
\hline 69 & 69149 & 0 & 0 & 0 & 0 & 0 & 0 & 0 & 0 & 0 & 0 & 0 & 0 & 0 & 0 & 0 & 0 & 0 \\
\hline 70 & 0 & 0 & 0 & 0 & 26880 & 0 & 0 & 0 & 0 & 0 & 0 & 13111 & 0 & 0 & 0 & 0 & 0 & 0 \\
\hline 71 & 32313 & 0 & 0 & 0 & 0 & 30799 & 0 & 0 & 0 & 0 & 0 & 0 & 14264 & 0 & 0 & 0 & 0 & 0 \\
\hline 73 & 26484 & 0 & 0 & 0 & 0 & 0 & 0 & 0 & 0 & 0 & 0 & 0 & 0 & 0 & 17918 & 20009 & 0 & 0 \\
\hline 74 & 0 & 0 & 0 & 23625 & 22961 & 35282 & 0 & 0 & 0 & 0 & 0 & 0 & 42099 & 0 & 0 & 0 & 0 & 0 \\
\hline 75 & 26707 & 32248 & 0 & 25611 & 27063 & 31516 & 0 & 0 & 0 & 0 & 0 & 32924 & 0 & 0 & 0 & 0 & 0 & 0 \\
\hline 76 & 30609 & 0 & 0 & 0 & 0 & 0 & 0 & 0 & 0 & 0 & 0 & 32216 & 0 & 0 & 0 & 0 & 0 & 0 \\
\hline 77 & 0 & 0 & 0 & 47075 & 48742 & 54559 & 0 & 0 & 37185 & 25951 & 24031 & 0 & 0 & 0 & 0 & 0 & 0 & 0 \\
\hline 78 & 19987 & 0 & 0 & 30559 & 55773 & 0 & 0 & 0 & 45915 & 27307 & 0 & 0 & 0 & 0 & 0 & 0 & 0 & 0 \\
\hline 79 & 0 & 0 & 0 & 0 & 0 & 0 & 0 & 0 & 0 & 0 & 0 & 0 & 0 & 0 & 0 & 0 & 0 & 0 \\
\hline 81 & 54227 & 33559 & 12307 & 0 & 30981 & 41379 & 10976 & 0 & 0 & 0 & 17459 & 0 & 26035 & 0 & 0 & 64092 & 0 & 0 \\
\hline 82 & 0 & 0 & 0 & 0 & 0 & 0 & 0 & 0 & 0 & 0 & $\begin{array}{c}0 \\
16390\end{array}$ & 0 & 0 & 0 & 27727 & 0 & 0 & 0 \\
\hline 83 & 20847 & 0 & 0 & 0 & 0 & 0 & 18338 & 0 & 0 & 0 & 3 & 0 & 0 & 0 & 0 & 17909 & 0 & 0 \\
\hline 84 & 23092 & 0 & 0 & 0 & 0 & 0 & 0 & 0 & 0 & 0 & 0 & 0 & 0 & 0 & 0 & 27178 & 0 & 0 \\
\hline 85 & 44289 & 0 & 0 & 0 & 0 & 0 & 0 & 0 & 0 & 0 & 0 & 0 & 0 & 0 & 0 & 19741 & 0 & 0 \\
\hline 86 & 0 & 0 & 0 & 0 & 0 & 0 & 0 & 0 & 0 & 0 & 0 & 0 & 0 & 0 & 0 & 22537 & 0 & 0 \\
\hline 87 & 0 & 0 & 20230 & 25924 & 53393 & 0 & 0 & 0 & 56718 & 92882 & 0 & 0 & 60933 & 12490 & 14256 & 0 & 0 & 0 \\
\hline 88 & 0 & 0 & 0 & 0 & 0 & 0 & 0 & 0 & 0 & 0 & 0 & 0 & 0 & 0 & 0 & 0 & 0 & 0 \\
\hline
\end{tabular}




\begin{tabular}{|c|c|c|c|c|c|c|c|c|c|c|c|c|c|c|c|c|c|}
\hline 89 & 0 & 0 & 0 & 0 & 0 & 0 & 0 & 0 & 0 & 0 & 0 & 0 & 0 & 0 & 0 & 0 & 0 \\
\hline 92 & 0 & 0 & 0 & 0 & 0 & 0 & 0 & 0 & 0 & 0 & 0 & 0 & 0 & 0 & 18964 & 0 & 0 \\
\hline 93 & 0 & 0 & 0 & 0 & 0 & 0 & 0 & 0 & 0 & 0 & 0 & 0 & 0 & 0 & 0 & 0 & 0 \\
\hline 94 & 0 & 0 & 0 & 0 & 0 & 0 & 0 & 0 & 0 & 0 & 0 & 40499 & 0 & 0 & 0 & 0 & 0 \\
\hline 95 & 0 & 0 & 0 & 0 & 0 & 0 & 0 & 0 & 0 & 0 & 14548 & 0 & 0 & 0 & 0 & 0 & 0 \\
\hline 98 & 0 & 0 & 0 & 49096 & 47680 & 44652 & 0 & 0 & 27973 & 26024 & 0 & 0 & 14818 & 0 & 0 & 0 & 0 \\
\hline 99 & 0 & 0 & 0 & 0 & 0 & 0 & 0 & 0 & 0 & 0 & 0 & 0 & 0 & 0 & 0 & 0 & 0 \\
\hline 101 & 46853 & 43424 & 0 & 0 & 0 & 0 & 0 & 0 & 0 & 0 & 0 & 0 & 0 & 0 & 0 & 0 & 0 \\
\hline 102 & 0 & 0 & 0 & 0 & 0 & 0 & 0 & 0 & 0 & 0 & 0 & 0 & 0 & 0 & 16872 & 0 & 0 \\
\hline 103 & 0 & 0 & 0 & 0 & 0 & 0 & 0 & 0 & 0 & 0 & 0 & 0 & 0 & 0 & 0 & 0 & 0 \\
\hline 104 & 83291 & 63234 & 0 & 0 & 0 & 40841 & 14188 & 0 & 0 & 0 & 20820 & 0 & 98878 & 0 & 0 & 0 & 0 \\
\hline 105 & 15750 & 20441 & 0 & 0 & 0 & 0 & 0 & 0 & 0 & 0 & 0 & 0 & 0 & 0 & 0 & 0 & 0 \\
\hline 106 & 0 & 20536 & 0 & 0 & 0 & 0 & 0 & 0 & 0 & 0 & 0 & 0 & 0 & 0 & 0 & 0 & 0 \\
\hline 107 & 0 & 0 & 0 & 0 & 0 & 0 & 0 & 0 & 0 & 0 & 0 & 23476 & 0 & 0 & 0 & 0 & 0 \\
\hline 108 & 0 & 0 & 0 & 0 & 0 & 0 & 0 & 0 & 0 & 0 & 0 & 19188 & 0 & 0 & 0 & 0 & 0 \\
\hline 109 & 0 & 0 & 0 & 0 & 0 & 0 & 0 & 0 & 0 & 0 & 0 & 16857 & 0 & 0 & 0 & 0 & 0 \\
\hline 114 & 0 & 0 & 0 & 0 & 0 & 0 & 0 & 0 & 0 & 0 & 0 & 0 & 0 & 0 & 0 & 0 & 0 \\
\hline 115 & 0 & 0 & 0 & 0 & 0 & 0 & 0 & 0 & 0 & 0 & 0 & 0 & 0 & 0 & 0 & 0 & 0 \\
\hline 116 & 0 & 0 & 0 & 0 & 0 & 0 & 0 & 0 & 0 & 0 & 0 & 0 & 0 & 0 & 0 & 0 & 0 \\
\hline 117 & 0 & 0 & 0 & 0 & 0 & 0 & 0 & 0 & 0 & 0 & 0 & 0 & 0 & 0 & 0 & 0 & 0 \\
\hline 118 & 0 & 0 & 0 & 0 & 0 & 0 & 0 & 0 & 0 & 0 & 0 & 0 & 0 & 0 & 0 & 0 & 0 \\
\hline 119 & 0 & 0 & 0 & 0 & 0 & 0 & 0 & 0 & 0 & 0 & 0 & 0 & 0 & 0 & 0 & 0 & 0 \\
\hline 120 & 0 & 0 & 0 & 0 & 0 & 0 & 0 & 0 & 0 & 0 & 0 & 0 & 0 & 0 & 0 & 0 & 0 \\
\hline 121 & 0 & 0 & 0 & 0 & 0 & 0 & 0 & 0 & 0 & 0 & 0 & 0 & 0 & 0 & 0 & 0 & 0 \\
\hline 122 & 0 & 0 & 0 & 0 & 0 & 0 & 0 & 0 & 0 & 0 & 0 & 0 & 0 & 0 & 22495 & 0 & 0 \\
\hline 124 & 0 & 0 & 0 & 0 & 0 & 0 & 0 & 0 & 0 & 0 & 0 & 0 & 0 & 0 & 0 & 0 & 0 \\
\hline 126 & 16180 & 19621 & 0 & 0 & 0 & 0 & 0 & 0 & 0 & 0 & 0 & 0 & 0 & 0 & 0 & 0 & 0 \\
\hline 127 & 18856 & 22430 & 0 & 0 & 0 & 0 & 0 & 0 & 0 & 0 & 0 & 0 & 15649 & 0 & 0 & 0 & 0 \\
\hline 128 & 29924 & 42761 & 0 & 0 & 0 & 0 & 0 & 0 & 0 & 0 & 0 & 0 & 0 & 0 & 0 & 0 & 0 \\
\hline 129 & 0 & 0 & 0 & 0 & 0 & 0 & 0 & 0 & 0 & 0 & 0 & 0 & 0 & 0 & 0 & 0 & 0 \\
\hline 130 & 0 & 0 & 0 & 0 & 0 & 0 & 0 & 0 & 0 & 0 & 0 & 0 & 0 & 0 & 0 & 0 & 0 \\
\hline 132 & 0 & 0 & 0 & 0 & 0 & 0 & 0 & 0 & 0 & 0 & 0 & 31384 & 0 & 0 & 0 & 0 & 0 \\
\hline 134 & 0 & 0 & 0 & 0 & 0 & 0 & 0 & 0 & 0 & 0 & 0 & 0 & 0 & 0 & 0 & 0 & 0 \\
\hline
\end{tabular}




\begin{tabular}{|c|c|c|c|c|c|c|c|c|c|c|c|c|c|c|c|c|c|}
\hline 135 & 0 & 0 & 0 & 0 & 0 & 0 & 0 & 0 & 0 & 0 & 0 & 0 & 0 & 0 & 0 & 0 & 0 \\
\hline 137 & 0 & 0 & 0 & 0 & 0 & 0 & $\begin{array}{l}11110 \\
10413\end{array}$ & 0 & 0 & 0 & $\begin{array}{l}32617 \\
51027\end{array}$ & 34048 & 0 & 0 & 0 & 0 & 0 \\
\hline 138 & 0 & 0 & 0 & 0 & 0 & 0 & 6 & 91672 & 0 & 0 & 2 & 0 & 0 & 0 & 0 & 0 & 0 \\
\hline 139 & 0 & 0 & 0 & 0 & 0 & 0 & 0 & 0 & 0 & 0 & 0 & 0 & 0 & 0 & 0 & 0 & 0 \\
\hline 142 & 0 & 0 & 0 & 0 & 0 & 0 & 0 & 14605 & 0 & 0 & 0 & 0 & 0 & 0 & 18310 & 0 & 0 \\
\hline 146 & 30306 & 57946 & 0 & 22753 & 0 & 22147 & 0 & 0 & 0 & 0 & 0 & 20104 & 18003 & 0 & 0 & 22537 & 0 \\
\hline 147 & 15480 & 0 & 0 & 0 & 0 & 24792 & 0 & 0 & 0 & 0 & 0 & 0 & 0 & 0 & 0 & 0 & 0 \\
\hline 149 & 0 & 0 & 0 & 0 & 0 & 0 & 0 & 0 & 0 & 0 & 0 & 0 & 0 & 0 & 0 & 0 & 0 \\
\hline 150 & 44464 & 50543 & 0 & 0 & 0 & 38196 & 0 & 0 & 0 & 0 & 0 & 0 & 0 & 0 & 0 & 0 & 0 \\
\hline 151 & 0 & 0 & 0 & 0 & 0 & 0 & 0 & 0 & 0 & 0 & 0 & 0 & 0 & 0 & 0 & 0 & 0 \\
\hline 152 & 0 & 20078 & 0 & 0 & 0 & 0 & 0 & 0 & 0 & 0 & 0 & 0 & 0 & 0 & 0 & 0 & 0 \\
\hline 153 & 0 & 0 & 0 & 0 & 0 & 0 & 0 & 0 & 0 & 0 & 0 & 44246 & 0 & 0 & 0 & 0 & 0 \\
\hline 156 & 18904 & 29912 & 0 & 0 & 0 & 0 & 0 & 0 & 0 & 0 & 0 & 0 & 0 & 0 & 0 & 0 & 0 \\
\hline 157 & 0 & 18263 & 0 & 0 & 0 & 0 & 0 & 0 & 0 & 0 & 0 & 0 & 0 & 0 & 0 & 0 & 0 \\
\hline 158 & 0 & 0 & 0 & 0 & 0 & 0 & 0 & 0 & 0 & 0 & 0 & 26015 & 0 & 0 & 0 & 0 & 0 \\
\hline 160 & 0 & 0 & 0 & 0 & 0 & 0 & 0 & 0 & 0 & 0 & 0 & 0 & 22850 & 0 & 0 & 0 & 0 \\
\hline 161 & 0 & 0 & 0 & 0 & 0 & 31696 & 0 & 0 & 0 & 0 & 0 & 0 & 0 & 0 & 0 & 0 & 0 \\
\hline 162 & 0 & 0 & 0 & 0 & 0 & 0 & 0 & 0 & 0 & 0 & 0 & 0 & 0 & 0 & 18049 & 0 & 0 \\
\hline 165 & 0 & 0 & 0 & 0 & 0 & 0 & 0 & 0 & 0 & 0 & 0 & 0 & 0 & 0 & 0 & 0 & 0 \\
\hline 167 & 0 & 0 & 0 & 55194 & 46545 & 0 & 0 & 0 & 0 & 0 & 0 & 0 & 0 & 0 & 0 & 0 & 0 \\
\hline 170 & 0 & 0 & 0 & 0 & 0 & 0 & 0 & 0 & 0 & 0 & 0 & 0 & 0 & 0 & 0 & 0 & 0 \\
\hline 171 & 0 & 0 & 0 & 0 & 0 & 0 & 0 & 0 & 0 & 0 & 0 & 0 & 0 & 0 & 0 & 0 & 0 \\
\hline 172 & 19334 & 29486 & 0 & 0 & 0 & 0 & 0 & 0 & 0 & 0 & 0 & 0 & 0 & 0 & 0 & 0 & 0 \\
\hline 175 & 0 & 0 & 0 & 0 & 0 & 0 & 0 & 0 & 0 & 0 & 0 & 15692 & 0 & 18328 & 0 & 0 & 0 \\
\hline 176 & 0 & 0 & 0 & 0 & 0 & 0 & 0 & 0 & 0 & 0 & 0 & 0 & 0 & 0 & 0 & 0 & 0 \\
\hline 177 & 27360 & 35232 & 0 & 0 & 0 & 31292 & 0 & 0 & 21462 & 0 & 0 & 33590 & 0 & 0 & 0 & 0 & 0 \\
\hline 178 & 23363 & 33685 & 0 & 0 & 0 & 0 & 0 & 0 & 0 & 0 & 0 & 0 & 0 & 0 & 0 & 0 & 0 \\
\hline 179 & 23920 & 35279 & 0 & 0 & 0 & 23536 & 0 & 0 & 27009 & 0 & 0 & 0 & 18972 & 0 & 0 & 0 & 0 \\
\hline 180 & 16610 & 24498 & 0 & 0 & 0 & 0 & 0 & 0 & 0 & 0 & 0 & 0 & 0 & 0 & 0 & 0 & 0 \\
\hline 182 & 0 & 0 & 0 & 0 & 0 & 0 & 0 & 0 & 0 & 0 & 0 & 0 & 0 & 0 & 20534 & 0 & 0 \\
\hline 184 & 0 & 0 & 0 & 0 & 0 & 0 & 0 & 0 & 0 & 0 & 0 & 0 & 0 & 0 & 0 & 0 & 0 \\
\hline 185 & 0 & 0 & 0 & 0 & 0 & 0 & 0 & 0 & 0 & 0 & 0 & 0 & 0 & 0 & 0 & 0 & 0 \\
\hline 187 & 0 & 0 & 25375 & 0 & 0 & 0 & 0 & 0 & 0 & 0 & 0 & 0 & 0 & 18226 & 0 & 0 & 0 \\
\hline
\end{tabular}




\begin{tabular}{|c|c|c|c|c|c|c|c|c|c|c|c|c|c|c|c|c|c|c|}
\hline & & & 43104 & & & & & & & & & & & 29765 & 26981 & & 11668 & \\
\hline 188 & 0 & 0 & 9 & 48573 & 0 & 0 & 0 & 0 & 0 & 0 & 0 & 16441 & 18142 & 0 & 4 & 0 & $\begin{array}{c}3 \\
11459\end{array}$ & 70707 \\
\hline 189 & 0 & 0 & 0 & 0 & 0 & 0 & 0 & 0 & 0 & 0 & 0 & 0 & 0 & 0 & 0 & 0 & 7 & 0 \\
\hline 190 & 0 & 0 & 0 & 0 & 0 & 0 & 0 & 0 & 0 & 0 & 0 & 0 & 0 & 0 & 0 & 0 & 0 & 0 \\
\hline 191 & 17470 & 32327 & 0 & 0 & 0 & 29768 & 0 & 0 & 0 & 0 & 0 & 0 & 15095 & 0 & 0 & 0 & 0 & 0 \\
\hline 192 & 0 & 0 & 0 & 0 & 0 & 0 & 0 & 0 & 0 & 0 & 0 & 14235 & 0 & 0 & 17918 & 0 & 0 & 0 \\
\hline 193 & 16387 & 17916 & 0 & 0 & 0 & 0 & 0 & 0 & 0 & 0 & 0 & 0 & 0 & 0 & 0 & 21641 & 0 & 0 \\
\hline 194 & 0 & 0 & 0 & 0 & 0 & 0 & 0 & 0 & 0 & 0 & 0 & 0 & 0 & 0 & 0 & 0 & 0 & 0 \\
\hline 197 & 18585 & 16606 & 0 & 0 & 0 & 0 & 0 & 0 & 0 & 0 & 0 & 0 & 0 & 0 & 0 & 0 & 0 & 0 \\
\hline 199 & 0 & 0 & 0 & 0 & 0 & 0 & 0 & 0 & 0 & 0 & 0 & 29261 & 0 & 0 & 0 & 0 & 0 & 0 \\
\hline 200 & 15766 & 0 & 0 & 0 & 0 & 25419 & 0 & 0 & 0 & 0 & 0 & 0 & 0 & 0 & 0 & 0 & 0 & 0 \\
\hline 201 & 0 & 35042 & 0 & 30106 & 25341 & 45234 & 12716 & 0 & $\begin{array}{l}49436 \\
12887\end{array}$ & 29763 & 0 & 0 & 24235 & 0 & 0 & 0 & 0 & 0 \\
\hline 202 & 34224 & 70195 & 0 & 63382 & 46032 & 74150 & 45911 & 47545 & 0 & 66234 & 13650 & 0 & 87246 & 0 & 21057 & 61685 & 0 & 0 \\
\hline 203 & 21420 & 0 & 0 & 0 & 0 & 23760 & 0 & 0 & 0 & 0 & 0 & 0 & 0 & 0 & 0 & 49808 & 0 & 0 \\
\hline 204 & 0 & 21199 & 0 & 0 & 0 & 0 & 0 & 0 & 0 & 0 & 0 & $\begin{array}{c}0 \\
12795\end{array}$ & 0 & 0 & 0 & 0 & 0 & 0 \\
\hline 205 & 0 & 0 & 0 & 0 & 0 & 0 & 0 & 0 & 0 & 0 & 0 & 0 & 0 & 0 & 0 & 0 & 0 & 0 \\
\hline 206 & 0 & 0 & 0 & 0 & 0 & 0 & 0 & 0 & 0 & 0 & 0 & 0 & 0 & 0 & 0 & 0 & 0 & 0 \\
\hline 210 & 0 & 0 & 0 & 0 & 0 & 0 & 0 & 0 & 0 & 0 & 0 & 0 & 0 & 0 & 0 & 0 & 0 & 0 \\
\hline 211 & 30020 & 62539 & 0 & 23555 & 0 & 38375 & 0 & 0 & 20739 & 0 & 0 & 0 & 0 & 0 & 0 & 0 & 0 & 0 \\
\hline 212 & 0 & 19731 & 0 & 0 & 27502 & 0 & 0 & 0 & 23536 & 32842 & 0 & 0 & 0 & 0 & 0 & 0 & 0 & 0 \\
\hline 216 & 0 & 0 & 0 & 0 & 0 & 0 & 0 & 0 & 0 & 0 & 0 & 0 & 0 & 0 & 0 & 0 & 0 & 0 \\
\hline 217 & 0 & 0 & 0 & 0 & 0 & 0 & 0 & 0 & 0 & 0 & 0 & 30801 & 0 & 0 & 0 & 0 & 0 & 0 \\
\hline 219 & 0 & 30496 & 0 & 0 & 0 & 22909 & 0 & 0 & 0 & 0 & 0 & 32674 & 0 & 0 & 0 & 0 & 11698 & 0 \\
\hline 221 & 0 & 0 & 0 & 0 & 0 & 0 & 0 & 0 & 0 & 0 & 0 & 0 & 0 & 0 & 0 & 0 & 0 & 0 \\
\hline 222 & 0 & 0 & 0 & 0 & 0 & 0 & 0 & 0 & 0 & 0 & 0 & 0 & 0 & 0 & 19618 & 0 & 0 & 0 \\
\hline 224 & 0 & 0 & 0 & 0 & 0 & 0 & 0 & 0 & 0 & 0 & 0 & 15442 & 0 & 0 & 0 & 0 & 0 & 0 \\
\hline 225 & 0 & 0 & 0 & 0 & 0 & 0 & 0 & 0 & 0 & 0 & 0 & 0 & 0 & 0 & 0 & 0 & 0 & 0 \\
\hline 226 & 0 & 0 & 0 & 23799 & 0 & 32368 & 0 & 0 & 0 & 0 & 0 & 0 & 0 & 0 & 0 & 0 & 0 & 0 \\
\hline 227 & 0 & 34348 & 0 & 0 & 0 & 0 & 0 & 0 & 0 & 0 & 0 & 0 & 0 & 0 & 0 & 0 & 0 & 0 \\
\hline 228 & 0 & 0 & 0 & 0 & 0 & 0 & 0 & 0 & 0 & 0 & 0 & 14235 & 0 & 46806 & 34789 & 0 & 79204 & 0 \\
\hline 229 & 0 & 0 & 0 & 0 & 0 & 0 & 0 & 0 & 0 & 0 & 0 & 0 & 0 & 0 & 0 & 0 & 0 & 16632 \\
\hline 230 & 0 & 0 & 0 & 0 & 0 & 0 & 0 & 0 & 0 & 0 & 0 & 0 & 0 & 0 & 0 & 0 & 0 & 0 \\
\hline
\end{tabular}




\begin{tabular}{|c|c|c|c|c|c|c|c|c|c|c|c|c|c|c|c|c|c|c|}
\hline 231 & 0 & 0 & 0 & 0 & 0 & 0 & 0 & 0 & 0 & 0 & 0 & 0 & 0 & 0 & 0 & 0 & 0 & 0 \\
\hline 232 & 0 & 0 & 0 & 0 & 0 & 0 & 0 & 0 & 0 & 0 & 0 & 0 & 0 & 0 & 0 & 0 & 0 & 0 \\
\hline 233 & 0 & 0 & 0 & 0 & 0 & 0 & 0 & 0 & 0 & 0 & 0 & 0 & 0 & 0 & 0 & 0 & 0 & 0 \\
\hline 234 & 0 & 0 & 0 & 0 & 0 & 0 & 0 & 0 & 0 & 0 & 0 & 0 & 0 & 0 & 0 & 0 & 0 & 0 \\
\hline 235 & 0 & 0 & 0 & 0 & 0 & 0 & 0 & 0 & 0 & 0 & 0 & 0 & 0 & 0 & 0 & 0 & 0 & 0 \\
\hline 236 & 0 & 0 & 0 & 0 & 0 & 0 & 0 & 0 & 0 & 0 & 0 & 0 & 0 & 0 & 0 & 0 & 0 & 0 \\
\hline 238 & 0 & 0 & 12047 & 0 & 0 & 0 & 0 & 0 & 0 & 0 & 0 & 54984 & 0 & 0 & 0 & 0 & 17063 & 0 \\
\hline 239 & 0 & 0 & 0 & 0 & 0 & 0 & 0 & 0 & 0 & 0 & 0 & 0 & 0 & 0 & 0 & 0 & 0 & 0 \\
\hline 240 & 0 & 0 & 0 & 0 & 0 & 0 & 0 & 0 & 0 & 0 & 0 & 0 & 0 & 0 & 0 & 0 & 0 & 0 \\
\hline 241 & 0 & 0 & $\begin{array}{c}0 \\
10310\end{array}$ & 0 & 0 & 0 & 0 & 0 & 0 & 0 & 0 & 0 & 0 & 34450 & 21841 & 0 & 0 & 0 \\
\hline 242 & 0 & 0 & 6 & 54810 & 28454 & 0 & 0 & 0 & 0 & 0 & 0 & 0 & 17449 & 57133 & 80957 & 37142 & $\begin{array}{c}0 \\
15121\end{array}$ & 58789 \\
\hline 243 & 0 & 0 & 0 & 0 & 0 & 0 & 0 & 0 & 0 & 0 & 0 & 0 & 0 & 0 & 0 & 0 & 8 & 0 \\
\hline 244 & 0 & 0 & 0 & 0 & 0 & 0 & 0 & 0 & 0 & 0 & 0 & 0 & 0 & 0 & 0 & 0 & 0 & 0 \\
\hline 250 & 0 & 0 & 0 & 0 & 0 & 0 & 0 & 0 & 0 & 0 & 0 & 0 & 0 & 0 & 0 & 0 & 0 & 0 \\
\hline 253 & 0 & 0 & 0 & 0 & 0 & 0 & 0 & 0 & 0 & 0 & 0 & 0 & 0 & 0 & 0 & 0 & 0 & 0 \\
\hline 254 & 0 & 0 & 0 & 0 & 0 & 0 & 0 & 0 & 0 & 0 & 0 & 0 & 0 & 0 & 0 & 0 & 0 & 0 \\
\hline 255 & 0 & 0 & 0 & 0 & 0 & 0 & 0 & 0 & 0 & 0 & 0 & 13028 & 0 & 0 & 0 & 0 & 0 & 0 \\
\hline 256 & 0 & 0 & 0 & 0 & 0 & 0 & 0 & 0 & 0 & 0 & 0 & 0 & 0 & 0 & 0 & 0 & 0 & 0 \\
\hline 257 & 0 & 0 & 0 & 0 & 0 & 0 & 0 & 0 & 0 & 0 & 0 & 0 & 0 & 0 & 0 & 0 & 0 & 0 \\
\hline 258 & 0 & 0 & 0 & 31674 & 24682 & 21653 & 0 & 0 & 22186 & 23275 & 0 & 0 & 0 & 0 & 0 & 0 & 0 & 0 \\
\hline 260 & 0 & 0 & 0 & 0 & 0 & 0 & 0 & 0 & 0 & 0 & 0 & 0 & 0 & 0 & 0 & 0 & 0 & 0 \\
\hline 261 & 0 & 0 & 0 & 0 & 0 & 0 & 0 & 19577 & 0 & 0 & 0 & 0 & 0 & 0 & 0 & 0 & 0 & 0 \\
\hline 262 & 0 & 0 & 0 & 0 & 0 & 0 & 0 & 30764 & 0 & 0 & 0 & 0 & 0 & 0 & 0 & 0 & 0 & 0 \\
\hline 264 & 0 & 0 & 0 & 0 & 0 & 0 & 0 & 0 & 0 & 0 & 0 & 0 & 0 & 46676 & 31520 & 0 & 0 & 0 \\
\hline 265 & 0 & 0 & 0 & 0 & 0 & 0 & 0 & 0 & 0 & 0 & 0 & 0 & 0 & 0 & 0 & 0 & 0 & 0 \\
\hline 266 & 0 & 0 & 20966 & 29548 & 40979 & 0 & 0 & 0 & 0 & 0 & 0 & 0 & 0 & $\begin{array}{l}33562 \\
10802\end{array}$ & 0 & 15555 & 21496 & 0 \\
\hline 268 & 0 & 0 & 0 & 44009 & 30615 & 0 & 0 & 0 & 45674 & 0 & 0 & 0 & 0 & 6 & 87758 & 91270 & 44073 & 12668 \\
\hline 270 & 0 & 0 & 0 & 25402 & 42297 & 0 & 0 & 0 & 0 & 0 & 0 & 0 & 0 & 51503 & 29950 & 33089 & 36771 & 11035 \\
\hline 272 & 0 & 0 & 0 & 0 & 0 & 0 & $\begin{array}{l}39620 \\
41654\end{array}$ & $\begin{array}{l}25171 \\
42355\end{array}$ & 0 & 0 & 0 & 0 & 0 & 0 & 0 & 0 & 0 & 0 \\
\hline 273 & 0 & 0 & 0 & 0 & 0 & 0 & 4 & 5 & 0 & 83938 & 0 & 0 & 0 & 19850 & 0 & 48083 & 0 & 0 \\
\hline 274 & 0 & 0 & 0 & 0 & 0 & 0 & 32646 & 34711 & 20884 & 79686 & 45692 & 0 & 0 & 20364 & 0 & 0 & 24957 & 10394 \\
\hline
\end{tabular}




\begin{tabular}{|c|c|c|c|c|c|c|c|c|c|c|c|c|c|c|c|c|c|c|}
\hline & & & & & & & 2 & 0 & & & & & & & & & 2 & 5 \\
\hline 275 & 0 & 0 & 0 & 0 & 0 & 0 & 0 & 0 & 22234 & 0 & 0 & 0 & 0 & 0 & 0 & 0 & 0 & 0 \\
\hline 276 & 0 & 26424 & 0 & 0 & 0 & 0 & 0 & 0 & 0 & 0 & 0 & 0 & 0 & 0 & 0 & 0 & 0 & 0 \\
\hline 277 & 0 & 0 & 0 & 38608 & 0 & 0 & 0 & 0 & 0 & 46368 & 0 & 0 & 17034 & 0 & 0 & 0 & 0 & 0 \\
\hline 278 & 0 & 0 & 0 & 0 & 0 & 0 & 0 & 0 & 0 & 0 & 0 & 0 & 0 & 0 & 0 & 0 & 0 & 0 \\
\hline 280 & 0 & 0 & 0 & 0 & 0 & 0 & 0 & 0 & 0 & 0 & 0 & 15900 & 0 & 0 & 0 & 0 & 0 & 0 \\
\hline 282 & 0 & 0 & 0 & 0 & 0 & 0 & 0 & 0 & 0 & 0 & 0 & 0 & 0 & 0 & 0 & 0 & 0 & 0 \\
\hline 283 & 0 & 0 & 0 & 0 & 0 & 0 & 0 & 0 & 0 & 0 & 0 & 0 & 0 & 0 & 0 & 0 & 0 & 0 \\
\hline 284 & 0 & 0 & 0 & 0 & 0 & 0 & 0 & 0 & 0 & 0 & 0 & 0 & 0 & 25452 & 14910 & 0 & 0 & 0 \\
\hline 285 & 0 & 0 & 0 & 0 & 0 & 0 & 0 & 0 & 0 & 0 & 0 & 0 & 0 & 0 & 0 & 0 & 0 & 0 \\
\hline 286 & 0 & 0 & 0 & 0 & 0 & 0 & 0 & 0 & 0 & 0 & 0 & 0 & 0 & 0 & 0 & 0 & 0 & 0 \\
\hline 289 & 0 & 0 & 0 & 0 & 0 & 0 & 0 & 0 & 0 & 0 & 0 & 0 & 0 & 0 & 0 & 0 & 0 & 0 \\
\hline 290 & 0 & 0 & 0 & 0 & 0 & 0 & 0 & 0 & 0 & 0 & 0 & 0 & 0 & 0 & 0 & 0 & 0 & 0 \\
\hline 291 & 0 & 0 & 0 & 0 & 0 & 0 & 0 & 0 & 0 & 0 & 0 & 0 & 0 & 0 & 0 & 0 & 0 & 0 \\
\hline 292 & 0 & 0 & 0 & 0 & 0 & 0 & 0 & 0 & 0 & 0 & 0 & 0 & 0 & 14167 & 0 & 0 & 0 & 0 \\
\hline 293 & 0 & 0 & 0 & 0 & 0 & 0 & 0 & 0 & 0 & 0 & 0 & 0 & 0 & 0 & 0 & 0 & 0 & 0 \\
\hline 294 & 0 & 0 & 0 & 0 & 0 & 0 & 0 & 0 & 0 & 0 & 0 & 0 & 0 & 0 & 0 & 0 & 0 & 0 \\
\hline 295 & 0 & 0 & 0 & 0 & 0 & 0 & 0 & 0 & 0 & 0 & 0 & 0 & 0 & 0 & 0 & 0 & 13300 & 0 \\
\hline 298 & 0 & 0 & 0 & 0 & 0 & 0 & 0 & 0 & 0 & 0 & 0 & 0 & 0 & 0 & 0 & 0 & 0 & 0 \\
\hline 301 & 0 & 0 & 0 & 0 & 0 & 0 & 0 & 0 & 0 & 0 & 0 & 0 & 0 & 0 & 0 & 0 & 0 & 0 \\
\hline 302 & 0 & 0 & 0 & 0 & 0 & 0 & 0 & 0 & 0 & 0 & 0 & 0 & 0 & 95825 & 87497 & 0 & 0 & 0 \\
\hline 303 & 0 & 0 & 34871 & 0 & 0 & 0 & 0 & 0 & 0 & 0 & 0 & 0 & $\begin{array}{c}0 \\
26229\end{array}$ & 64581 & 52707 & 0 & 0 & 0 \\
\hline 304 & 0 & 0 & 13604 & 0 & 0 & 0 & 0 & 0 & 0 & 0 & 0 & 0 & 1 & 0 & 0 & 0 & 59608 & 18159 \\
\hline 305 & 0 & 0 & 0 & 0 & 23474 & 0 & 0 & 0 & 26478 & 44645 & 0 & 0 & 0 & 19910 & 0 & 0 & 0 & 0 \\
\hline 306 & 0 & 0 & 11872 & 46273 & 30432 & 0 & 0 & 0 & 0 & 44278 & 0 & 0 & 58302 & 0 & 0 & 0 & 0 & 0 \\
\hline 307 & 0 & 0 & 0 & 0 & 0 & 0 & 0 & 0 & 0 & 0 & 0 & 0 & 0 & 0 & 0 & 0 & 0 & 0 \\
\hline 310 & 0 & 0 & 0 & 0 & 0 & 0 & 0 & 0 & 0 & 0 & 0 & 0 & 0 & 0 & 0 & 0 & 0 & 0 \\
\hline 311 & 0 & 0 & 0 & 0 & 0 & 0 & 0 & 0 & 0 & 0 & 0 & 0 & 0 & 0 & 0 & 0 & 0 & 0 \\
\hline 312 & 0 & 0 & 0 & 0 & 0 & 0 & 0 & 0 & 0 & 0 & 0 & 0 & 0 & 0 & 0 & 0 & 0 & 0 \\
\hline 313 & 0 & 0 & 0 & 0 & 0 & 0 & 0 & 0 & 0 & 0 & 0 & 0 & 0 & 0 & 0 & 0 & 0 & 0 \\
\hline 315 & 0 & 0 & 0 & 0 & 0 & 0 & 0 & 0 & 0 & 0 & 0 & 0 & 0 & 0 & 0 & 0 & 0 & 0 \\
\hline 317 & 0 & 0 & 0 & 0 & 0 & 0 & 0 & 0 & 0 & 0 & 0 & 0 & 0 & 0 & 0 & 0 & 0 & 0 \\
\hline 318 & 0 & 0 & 0 & 0 & 0 & 0 & 0 & 0 & 0 & 0 & 0 & 0 & 0 & 0 & 0 & 0 & 0 & 0 \\
\hline
\end{tabular}




\begin{tabular}{|c|c|c|c|c|c|c|c|c|c|c|c|c|c|c|c|c|c|c|}
\hline 320 & 0 & 0 & 0 & 0 & 0 & 0 & 0 & 0 & 0 & 0 & 0 & 0 & 0 & 0 & 0 & 0 & 0 & 0 \\
\hline 322 & 0 & 0 & 0 & 0 & 0 & 0 & 0 & 0 & 0 & 0 & 0 & 0 & 0 & 0 & 14910 & 0 & 0 & 0 \\
\hline 329 & 0 & 0 & 0 & 0 & 0 & 0 & 0 & 0 & 0 & 0 & 0 & 48033 & 0 & 0 & 0 & 0 & 0 & 0 \\
\hline 332 & 0 & 0 & 0 & 0 & 0 & 0 & 0 & 0 & 0 & 0 & 0 & 0 & 0 & 0 & 0 & 0 & 0 & 0 \\
\hline 358 & 0 & 0 & 0 & 0 & 0 & 0 & 0 & 0 & 0 & 0 & 0 & 0 & 0 & 0 & 0 & 0 & 0 & 0 \\
\hline 359 & 0 & 0 & 0 & 0 & 0 & 0 & 0 & 0 & 0 & 0 & 0 & 0 & 0 & 0 & 0 & 0 & 0 & 0 \\
\hline 362 & 0 & 0 & 0 & 0 & 0 & 0 & 0 & 0 & 0 & 0 & 0 & 0 & 0 & 0 & 0 & 0 & 0 & 0 \\
\hline 386 & 0 & 0 & 0 & 0 & 0 & 0 & 0 & 0 & 0 & 0 & 0 & 0 & 0 & 0 & 0 & 0 & 0 & 0 \\
\hline 402 & 0 & 0 & 0 & 0 & 0 & 0 & 0 & 0 & 0 & 0 & 0 & 14610 & 0 & 0 & 0 & 0 & 0 & 0 \\
\hline 410 & 0 & 0 & 0 & 0 & 0 & 0 & 0 & 0 & 0 & 0 & 0 & 0 & 0 & 0 & 0 & 0 & 0 & 0 \\
\hline 430 & 0 & 0 & 0 & 80525 & 49328 & 84282 & 0 & 0 & 50304 & 47797 & 0 & 0 & 69519 & 0 & 0 & 16090 & 0 & 0 \\
\hline 439 & 0 & 0 & 0 & 0 & 0 & 22102 & 0 & 0 & 0 & 0 & 0 & 0 & 0 & 0 & 0 & 0 & 0 & 0 \\
\hline 451 & 0 & 0 & 0 & 0 & 0 & 0 & 0 & 0 & 0 & 0 & 0 & 46826 & 0 & 0 & 0 & 0 & 0 & 0 \\
\hline 459 & 0 & 0 & 0 & 0 & 0 & 0 & 0 & 0 & 0 & 0 & 0 & 22144 & 0 & 0 & 0 & 0 & 0 & 0 \\
\hline 476 & 0 & 0 & 0 & 0 & 0 & 0 & 0 & 0 & 0 & 0 & 0 & 0 & 0 & 0 & 0 & 0 & 15386 & 0 \\
\hline 502 & 0 & 0 & 13746 & 0 & 0 & 0 & 0 & 0 & 0 & 0 & 0 & 0 & 0 & 0 & 13602 & 0 & 0 & 0 \\
\hline 503 & 0 & 0 & 0 & 0 & 0 & 0 & 0 & 0 & 0 & 0 & 0 & 0 & 0 & 0 & 0 & 0 & 0 & 0 \\
\hline 504 & 0 & 0 & 30311 & 0 & 0 & 0 & 0 & 0 & 0 & 19830 & 0 & 0 & 0 & 0 & 0 & 0 & 0 & 0 \\
\hline 506 & 0 & 0 & 0 & 0 & 0 & 0 & 0 & 0 & 0 & 28810 & 0 & 0 & 0 & 0 & 0 & 0 & 0 & 0 \\
\hline 507 & 0 & 0 & 81672 & 0 & 25012 & 0 & 0 & 0 & 0 & 56594 & 0 & 0 & 0 & 0 & 0 & 0 & 0 & 11302 \\
\hline 508 & 0 & 0 & 44233 & 0 & 0 & 0 & 0 & 0 & 0 & 0 & 0 & 0 & 0 & 0 & 0 & 0 & 33567 & 0 \\
\hline 509 & 0 & 0 & 0 & 49305 & 35742 & 0 & 0 & 0 & 34147 & $\begin{array}{l}92552 \\
11043\end{array}$ & 0 & 0 & 0 & 0 & 0 & 0 & 0 & 0 \\
\hline 511 & 0 & 0 & 81044 & 48155 & 35266 & 25867 & 0 & 0 & 54500 & 9 & 0 & 0 & 0 & 15002 & 0 & 0 & 16988 & 0 \\
\hline 512 & 0 & 0 & 0 & 0 & 0 & 0 & 0 & 0 & 0 & 0 & 0 & 0 & 0 & 0 & 0 & 0 & 0 & 0 \\
\hline 513 & 0 & 0 & 19535 & 0 & 0 & 0 & 0 & 0 & 0 & 0 & 0 & 0 & 0 & 0 & 0 & 0 & 0 & 0 \\
\hline 515 & 0 & 0 & 13302 & 0 & 0 & 0 & 0 & 0 & 0 & 0 & 0 & 0 & 0 & 0 & 0 & 0 & 0 & 0 \\
\hline 518 & 0 & 0 & 18824 & 0 & 0 & 0 & 0 & 0 & 0 & 0 & 0 & 0 & 0 & 0 & 0 & 0 & 0 & 0 \\
\hline 526 & 0 & 0 & 0 & 0 & 0 & 0 & 0 & 0 & 0 & 0 & 0 & 0 & 0 & 0 & 0 & 0 & 0 & 0 \\
\hline 528 & 0 & 0 & 0 & 0 & 0 & 0 & 0 & 0 & 0 & 0 & 0 & 13652 & 0 & 0 & 0 & 0 & 0 & 0 \\
\hline 530 & 0 & 0 & 0 & 0 & 0 & 0 & 0 & 0 & 0 & 0 & 0 & 15650 & 0 & 0 & 0 & 0 & 18777 & 0 \\
\hline
\end{tabular}


Appendix 5. HaeIII Macro TRF results used for analysis in peak aera. Noise peaks have been truncated.

\begin{tabular}{|c|c|c|c|c|c|c|c|c|c|c|c|}
\hline $\mathrm{BP}$ & H-7-844 & H-7-255 & H-7-212 & H-7-209 & H-7-205 & H-7-190 & H-7-179 & H-7-111 & H-5-111 & H-5-179 & H-5-190 \\
\hline 56 & 0 & 0 & 29403 & 0 & 0 & 26955 & 0 & 0 & 100704 & 0 & 50029 \\
\hline 57 & 0 & 0 & 0 & 0 & 21103 & 0 & 0 & 0 & 0 & 0 & 33197 \\
\hline 58 & 0 & 0 & 0 & 0 & 0 & 0 & 0 & 0 & 0 & 0 & 0 \\
\hline 59 & 22497 & 0 & 0 & 0 & 21021 & 15895 & 38577 & 94764 & 56170 & 0 & 0 \\
\hline 61 & 0 & 0 & 13455 & 0 & 18361 & 0 & 0 & 0 & 0 & 0 & 30820 \\
\hline 62 & 0 & 0 & 15494 & 0 & 0 & 0 & 0 & 0 & 0 & 0 & 0 \\
\hline 63 & 0 & 0 & 0 & 0 & 16881 & 0 & 0 & 0 & 0 & 15829 & 23300 \\
\hline 64 & 15080 & 0 & 0 & 0 & 0 & 0 & 18912 & 0 & 86796 & 18942 & 28288 \\
\hline 66 & 0 & 0 & 0 & 0 & 31313 & 0 & 0 & 0 & 0 & 0 & 0 \\
\hline 67 & 0 & 0 & 0 & 23853 & 0 & 0 & 16529 & 0 & 0 & 0 & 19832 \\
\hline 68 & 0 & 80319 & 54144 & 170015 & 144941 & 28269 & 126490 & 0 & 74825 & 0 & 67134 \\
\hline 69 & 0 & 0 & 0 & 0 & 0 & 0 & 0 & 0 & 0 & 0 & 0 \\
\hline 70 & 0 & 0 & 0 & 0 & 0 & 0 & 0 & 0 & 0 & 0 & 0 \\
\hline 71 & 15328 & 0 & 0 & 12967 & 25295 & 21965 & 19634 & 0 & 39737 & 17968 & 80382 \\
\hline 73 & 0 & 0 & 0 & 0 & 16755 & 0 & 0 & 0 & 0 & 0 & 0 \\
\hline 74 & 0 & 0 & 0 & 0 & 0 & 0 & 0 & 0 & 0 & 0 & 24664 \\
\hline 75 & 15328 & 0 & 0 & 0 & 18409 & 21229 & 0 & 0 & 42873 & 20820 & 17884 \\
\hline 76 & 0 & 0 & 0 & 0 & 0 & 0 & 0 & 0 & 0 & 0 & 0 \\
\hline 77 & 0 & 0 & 0 & 0 & 0 & 0 & 0 & 0 & 0 & 15971 & 0 \\
\hline 78 & 0 & 0 & 0 & 0 & 0 & 0 & 0 & 0 & 0 & 16019 & 42587 \\
\hline 79 & 0 & 0 & 0 & 0 & 20404 & 27941 & 0 & 0 & 40161 & 25787 & 16014 \\
\hline 81 & 47219 & 0 & 0 & 0 & 23270 & 54599 & 33622 & 43802 & 35742 & 45229 & 28677 \\
\hline 82 & 0 & 0 & 0 & 0 & 0 & 0 & 0 & 0 & 0 & 0 & 0 \\
\hline 83 & 0 & 0 & 0 & 0 & 0 & 0 & 0 & 0 & 0 & 0 & 0 \\
\hline 84 & 0 & 0 & 0 & 0 & 0 & 0 & 0 & 0 & 0 & 0 & 0 \\
\hline 85 & 0 & 0 & 0 & 0 & 0 & 0 & 16936 & 0 & 0 & 0 & 0 \\
\hline 86 & 0 & 0 & 0 & 0 & 20874 & 0 & 0 & 0 & 0 & 0 & 0 \\
\hline 87 & 0 & 0 & 0 & 0 & 0 & 0 & 0 & 0 & 0 & 0 & 24430 \\
\hline 88 & 0 & 0 & 0 & 0 & 0 & 0 & 0 & 0 & 36718 & 0 & 0 \\
\hline 89 & 0 & 0 & 0 & 0 & 0 & 0 & 0 & 0 & 0 & 0 & 0 \\
\hline 92 & 0 & 0 & 0 & 0 & 0 & 0 & 0 & 0 & 0 & 0 & 0 \\
\hline
\end{tabular}




\begin{tabular}{|c|c|c|c|c|c|c|c|c|c|c|}
\hline 93 & 49691 & 0 & 0 & 0 & 0 & 28535 & 29984 & 78101 & 37698 & 29709 \\
\hline 94 & 0 & 0 & 0 & 0 & 0 & 15425 & 0 & 0 & 0 & 0 \\
\hline 95 & 0 & 0 & 0 & 0 & 0 & 0 & 0 & 0 & 0 & 0 \\
\hline 98 & 13844 & 0 & 0 & 16329 & 0 & 15425 & 0 & 0 & 0 & 17160 \\
\hline 99 & 18047 & 0 & 0 & 0 & 0 & 15019 & 0 & 0 & 0 & 21319 \\
\hline 101 & 62052 & 0 & 0 & 0 & 0 & 35904 & 0 & 33677 & 0 & 23197 \\
\hline 102 & 0 & 0 & 0 & 0 & 0 & 0 & 0 & 0 & 0 & 0 \\
\hline 103 & 0 & 0 & 0 & 0 & 0 & 21761 & 0 & 0 & 0 & 0 \\
\hline 104 & 22744 & 0 & 0 & 0 & 0 & 41160 & 0 & 33390 & 0 & 25312 \\
\hline 105 & 0 & 0 & 0 & 0 & 0 & 0 & 0 & 0 & 0 & 0 \\
\hline 106 & 0 & 0 & 0 & 0 & 0 & 24671 & 0 & 0 & 0 & 24955 \\
\hline 107 & 0 & 0 & 0 & 0 & 0 & 0 & 0 & 0 & 0 & 16494 \\
\hline 108 & 0 & 0 & 0 & 0 & 0 & 0 & 0 & 0 & 0 & 0 \\
\hline 109 & 12855 & 0 & 0 & 0 & 0 & 25312 & 0 & 0 & 0 & 0 \\
\hline 114 & 0 & 0 & 0 & 0 & 0 & 0 & 0 & 0 & 0 & 0 \\
\hline 115 & 0 & 0 & 0 & 0 & 0 & 0 & 0 & 0 & 0 & 0 \\
\hline 116 & 0 & 0 & 0 & 21532 & 0 & 17131 & 0 & 0 & 0 & 0 \\
\hline 117 & 0 & 0 & 0 & 0 & 0 & 0 & 0 & 38811 & 0 & 0 \\
\hline 118 & 0 & 0 & 0 & 0 & 0 & 0 & 0 & 0 & 0 & 0 \\
\hline 119 & 0 & 0 & 0 & 0 & 0 & 0 & 0 & 0 & 0 & 0 \\
\hline 120 & 0 & 0 & 0 & 0 & 0 & 15629 & 42153 & 25210 & 0 & 0 \\
\hline 121 & 0 & 0 & 0 & 0 & 0 & 0 & 0 & 0 & 0 & 0 \\
\hline 122 & 0 & 0 & 0 & 0 & 0 & 0 & 24464 & 0 & 0 & 0 \\
\hline 124 & 13103 & 0 & 0 & 0 & 0 & 20369 & 0 & 0 & 0 & 18847 \\
\hline 126 & 0 & 0 & 0 & 0 & 0 & 18961 & 0 & 0 & 0 & 28639 \\
\hline 127 & 0 & 0 & 0 & 0 & 0 & 0 & 0 & 33166 & 0 & 0 \\
\hline 128 & 39061 & 0 & 0 & 0 & 0 & 30678 & 0 & 42383 & 0 & 25835 \\
\hline 129 & 0 & 0 & 0 & 0 & 0 & 0 & 0 & 0 & 0 & 41117 \\
\hline 130 & 0 & 0 & 0 & 0 & 0 & 0 & 0 & 0 & 0 & 0 \\
\hline 132 & 0 & 0 & 0 & 0 & 0 & 0 & 0 & 0 & 0 & 0 \\
\hline 134 & 0 & 0 & 0 & 0 & 0 & 0 & 0 & 24205 & 0 & 0 \\
\hline 135 & 0 & 0 & 0 & 0 & 0 & 0 & 0 & 0 & 0 & 15092 \\
\hline 137 & 0 & 0 & 0 & 0 & 0 & 0 & 0 & 0 & 0 & 0 \\
\hline
\end{tabular}




\begin{tabular}{|c|c|c|c|c|c|c|c|c|c|c|c|}
\hline 138 & 0 & 0 & 0 & 0 & 0 & 0 & 0 & 0 & 0 & 0 & 113852 \\
\hline 139 & 0 & 0 & 0 & 0 & 0 & 0 & 0 & 0 & 0 & 0 & 0 \\
\hline 142 & 0 & 0 & 0 & 0 & 0 & 0 & 0 & 0 & 0 & 0 & 0 \\
\hline 146 & 0 & 0 & 0 & 0 & 0 & 0 & 0 & 0 & 0 & 21557 & 0 \\
\hline 147 & 102101 & 0 & 0 & 0 & 0 & 38751 & 0 & 33485 & 33481 & 27760 & 0 \\
\hline 149 & 0 & 0 & 0 & 0 & 0 & 0 & 0 & 0 & 0 & 0 & 0 \\
\hline 150 & 34858 & 0 & 0 & 0 & 0 & 27174 & 0 & 0 & 28634 & 40618 & 0 \\
\hline 151 & 36341 & 0 & 0 & 0 & 0 & 0 & 0 & 0 & 0 & 0 & 0 \\
\hline 152 & 0 & 0 & 0 & 0 & 0 & 0 & 0 & 0 & 0 & 0 & 0 \\
\hline 153 & 0 & 0 & 0 & 0 & 0 & 0 & 0 & 0 & 0 & 0 & 0 \\
\hline 156 & 0 & 0 & 0 & 0 & 0 & 0 & 0 & 23791 & 24996 & 21414 & 0 \\
\hline 157 & 0 & 0 & 0 & 0 & 0 & 0 & 0 & 0 & 0 & 0 & 0 \\
\hline 158 & 0 & 0 & 0 & 0 & 0 & 0 & 0 & 0 & 0 & 0 & 0 \\
\hline 160 & 0 & 0 & 0 & 0 & 0 & 0 & 0 & 0 & 0 & 0 & 0 \\
\hline 161 & 0 & 0 & 0 & 0 & 0 & 0 & 0 & 0 & 0 & 0 & 0 \\
\hline 162 & 0 & 0 & 0 & 0 & 0 & 0 & 0 & 0 & 0 & 0 & 0 \\
\hline 165 & 0 & 0 & 0 & 0 & 0 & 0 & 0 & 0 & 0 & 0 & 0 \\
\hline 167 & 0 & 0 & 0 & 0 & 0 & 0 & 0 & 0 & 0 & 0 & 0 \\
\hline 170 & 0 & 0 & 0 & 0 & 0 & 0 & 0 & 0 & 0 & 0 & 0 \\
\hline 171 & 0 & 0 & 0 & 0 & 0 & 0 & 0 & 0 & 0 & 0 & 0 \\
\hline 172 & 0 & 0 & 0 & 0 & 0 & 0 & 0 & 0 & 0 & 0 & 0 \\
\hline 175 & 0 & 0 & 0 & 0 & 0 & 0 & 0 & 0 & 0 & 0 & 0 \\
\hline 176 & 0 & 0 & 0 & 0 & 0 & 0 & 0 & 0 & 0 & 0 & 0 \\
\hline 177 & 0 & 0 & 24330 & 0 & 0 & 0 & 0 & 0 & 0 & 17231 & 0 \\
\hline 178 & 0 & 0 & 0 & 0 & 0 & 0 & 0 & 0 & 0 & 0 & 0 \\
\hline 179 & 0 & 0 & 28423 & 0 & 0 & 0 & 0 & 0 & 0 & 0 & 0 \\
\hline 180 & 0 & 0 & 0 & 0 & 0 & 23201 & 0 & 29355 & 0 & 29471 & 0 \\
\hline 182 & 0 & 0 & 0 & 0 & 0 & 0 & 0 & 0 & 0 & 0 & 0 \\
\hline 184 & 0 & 0 & 0 & 0 & 0 & 0 & 0 & 0 & 0 & 0 & 0 \\
\hline 185 & 0 & 0 & 0 & 0 & 0 & 0 & 0 & 0 & 0 & 0 & 0 \\
\hline 187 & 0 & 0 & 0 & 0 & 0 & 0 & 0 & 0 & 0 & 0 & 0 \\
\hline 188 & 28183 & 0 & 0 & 0 & 0 & 0 & 0 & 0 & 0 & 0 & 0 \\
\hline 189 & 0 & 0 & 0 & 0 & 0 & 18132 & 22990 & 62713 & 0 & 21509 & 0 \\
\hline
\end{tabular}




\begin{tabular}{|c|c|c|c|c|c|c|c|c|c|c|c|}
\hline 190 & 62546 & 0 & 12432 & 0 & 0 & 0 & 0 & 0 & 0 & 0 & 15391 \\
\hline 191 & 0 & 0 & 0 & 0 & 0 & 19055 & 0 & 41362 & 38893 & 16138 & 0 \\
\hline 192 & 0 & 0 & 12309 & 0 & 0 & 0 & 0 & 0 & 0 & 0 & 0 \\
\hline 193 & 0 & 0 & 31875 & 13848 & 0 & 19821 & 0 & 26437 & 0 & 17350 & 0 \\
\hline 194 & 0 & 0 & 379454 & 0 & 0 & 0 & 103218 & 0 & 0 & 0 & 24002 \\
\hline 197 & 0 & 0 & 11307 & 0 & 0 & 19602 & 17564 & 0 & 35403 & 31277 & 29534 \\
\hline 199 & 0 & 0 & 0 & 18330 & 0 & 0 & 0 & 0 & 0 & 0 & 0 \\
\hline 200 & 19530 & 0 & 0 & 0 & 0 & 0 & 0 & 33549 & 0 & 32537 & 0 \\
\hline 201 & 80099 & 0 & 0 & 228688 & 0 & 40143 & 0 & 31939 & 35345 & 36554 & 18157 \\
\hline 202 & 227441 & 18439 & 0 & 0 & 0 & 138045 & 35629 & 34346 & 56699 & 81331 & 48743 \\
\hline 203 & 23733 & 0 & 0 & 19691 & 0 & 45415 & 0 & 0 & 39181 & 41046 & 19443 \\
\hline 204 & 0 & 0 & 0 & 0 & 0 & 0 & 0 & 0 & 0 & 0 & 0 \\
\hline 205 & 0 & 0 & 0 & 0 & 0 & 0 & 0 & 0 & 0 & 0 & 0 \\
\hline 206 & 0 & 0 & 0 & 0 & 0 & 0 & 0 & 0 & 0 & 0 & 0 \\
\hline 210 & 0 & 0 & 0 & 0 & 0 & 0 & 0 & 26007 & 0 & 21272 & 0 \\
\hline 211 & 14339 & 17110 & 0 & 0 & 0 & 16833 & 16591 & 72137 & 35053 & 36958 & 0 \\
\hline 212 & 0 & 0 & 0 & 0 & 0 & 0 & 21014 & 25720 & 0 & 0 & 0 \\
\hline 216 & 0 & 0 & 0 & 0 & 0 & 0 & 0 & 0 & 0 & 0 & 0 \\
\hline 217 & 0 & 0 & 0 & 0 & 0 & 0 & 0 & 0 & 0 & 0 & 0 \\
\hline 219 & 0 & 0 & 0 & 0 & 0 & 0 & 0 & 0 & 0 & 0 & 0 \\
\hline 221 & 0 & 0 & 16568 & 0 & 0 & 0 & 0 & 0 & 0 & 0 & 0 \\
\hline 222 & 0 & 0 & 0 & 0 & 0 & 0 & 0 & 0 & 0 & 0 & 0 \\
\hline 224 & 0 & 0 & 0 & 17770 & 21789 & 0 & 22456 & 0 & 29618 & 0 & 29924 \\
\hline 225 & 0 & 0 & 19869 & 22893 & 0 & 0 & 23146 & 0 & 0 & 0 & 0 \\
\hline 226 & 0 & 30239 & 0 & 0 & 41999 & 0 & 0 & 0 & 0 & 0 & 0 \\
\hline 227 & 0 & 20361 & 24258 & 33459 & 0 & 0 & 32995 & 0 & 0 & 0 & 0 \\
\hline 228 & 0 & 0 & 36091 & 54350 & 48876 & 0 & 33120 & 0 & 0 & 0 & 25015 \\
\hline 229 & 0 & 38493 & 111365 & 87729 & 105027 & 16567 & 62947 & 0 & 29665 & 0 & 81785 \\
\hline 230 & 0 & 0 & 0 & 0 & 0 & 0 & 31395 & 0 & 0 & 0 & 34327 \\
\hline 231 & 0 & 0 & 24762 & 0 & 71015 & 0 & 0 & 0 & 0 & 0 & 0 \\
\hline 232 & 0 & 0 & 58799 & 0 & 0 & 0 & 17626 & 24971 & 0 & 0 & 0 \\
\hline 233 & 0 & 18754 & 0 & 0 & 37997 & 0 & 0 & 0 & 26817 & 0 & 45704 \\
\hline 234 & 0 & 0 & 0 & 0 & 0 & 0 & 32681 & 58679 & 0 & 0 & 0 \\
\hline
\end{tabular}




\begin{tabular}{|c|c|c|c|c|c|c|c|c|c|c|}
\hline 235 & 0 & 26178 & 12950 & 17930 & 24203 & 0 & 15556 & 0 & 0 & 0 \\
\hline 236 & 0 & 20240 & 0 & 0 & 0 & 0 & 0 & 0 & 0 & 0 \\
\hline 238 & 0 & 0 & 0 & 26895 & 30018 & 0 & 0 & 0 & 0 & 0 \\
\hline 239 & 0 & 72994 & 11769 & 0 & 50038 & 0 & 34186 & 0 & 0 & 0 \\
\hline 240 & 0 & 0 & 0 & 0 & 0 & 0 & 0 & 0 & 0 & 0 \\
\hline 241 & 0 & 24857 & 0 & 0 & 0 & 0 & 0 & 0 & 0 & 0 \\
\hline 242 & 0 & 0 & 0 & 0 & 0 & 0 & 0 & 0 & 0 & 0 \\
\hline 243 & 0 & 0 & 0 & 0 & 0 & 0 & 0 & 0 & 0 & 16708 \\
\hline 244 & 0 & 0 & 0 & 0 & 0 & 0 & 0 & 0 & 0 & 0 \\
\hline 250 & 0 & 0 & 0 & 0 & 0 & 0 & 0 & 0 & 0 & 0 \\
\hline 253 & 0 & 0 & 0 & 18090 & 0 & 0 & 0 & 0 & 0 & 0 \\
\hline 254 & 0 & 30283 & 0 & 44585 & 0 & 0 & 0 & 0 & 0 & 0 \\
\hline 255 & 0 & 18189 & 0 & 0 & 0 & 0 & 0 & 0 & 0 & 0 \\
\hline 256 & 0 & 0 & 0 & 0 & 0 & 0 & 0 & 0 & 0 & 0 \\
\hline 257 & 0 & 0 & 0 & 20411 & 0 & 0 & 0 & 0 & 0 & 0 \\
\hline 258 & 0 & 48475 & 0 & 0 & 0 & 0 & 0 & 0 & 34792 & 0 \\
\hline 260 & 0 & 0 & 0 & 0 & 0 & 0 & 0 & 0 & 0 & 0 \\
\hline 261 & 0 & 32125 & 0 & 0 & 0 & 0 & 0 & 0 & 0 & 0 \\
\hline 262 & 0 & 0 & 0 & 0 & 0 & 0 & 0 & 0 & 0 & 0 \\
\hline 264 & 0 & 0 & 0 & 0 & 0 & 0 & 0 & 0 & 0 & 0 \\
\hline 265 & 0 & 20908 & 0 & 0 & 20546 & 0 & 0 & 0 & 0 & 0 \\
\hline 266 & 0 & 20014 & 0 & 52990 & 31659 & 0 & 19759 & 0 & 0 & 0 \\
\hline 268 & 0 & 0 & 0 & 0 & 0 & 0 & 0 & 0 & 0 & 0 \\
\hline 270 & 0 & 28489 & 0 & 0 & 0 & 0 & 0 & 0 & 0 & 0 \\
\hline 272 & 0 & 36556 & 0 & 0 & 0 & 0 & 0 & 0 & 0 & 0 \\
\hline 273 & 0 & 40235 & 0 & 0 & 17265 & 0 & 23523 & 0 & 0 & 0 \\
\hline 274 & 0 & 18134 & 0 & 0 & 0 & 0 & 0 & 0 & 0 & 0 \\
\hline 275 & 0 & 32829 & 0 & 0 & 0 & 0 & 0 & 28000 & 0 & 0 \\
\hline 276 & 0 & 0 & 0 & 0 & 0 & 0 & 0 & 0 & 0 & 0 \\
\hline 277 & 0 & 35174 & 0 & 0 & 0 & 0 & 0 & 0 & 0 & 0 \\
\hline 278 & 0 & 0 & 0 & 0 & 0 & 0 & 0 & 0 & 0 & 0 \\
\hline 280 & 0 & 0 & 0 & 0 & 0 & 0 & 0 & 0 & 0 & 0 \\
\hline 282 & 0 & 0 & 0 & 0 & 0 & 0 & 0 & 0 & 0 & 0 \\
\hline
\end{tabular}




\begin{tabular}{|c|c|c|c|c|c|c|c|c|c|c|}
\hline 283 & 0 & 20339 & 0 & 26655 & 36486 & 22246 & 33622 & 0 & 0 & 0 \\
\hline 284 & 0 & 0 & 0 & 0 & 0 & 0 & 0 & 0 & 0 & 0 \\
\hline 285 & 0 & 16844 & 0 & 0 & 0 & 0 & 0 & 0 & 0 & 0 \\
\hline 286 & 0 & 25211 & 0 & 0 & 0 & 0 & 0 & 0 & 0 & 0 \\
\hline 289 & 0 & 0 & 0 & 0 & 0 & 0 & 0 & 0 & 0 & 0 \\
\hline 290 & 0 & 27679 & 0 & 0 & 0 & 0 & 0 & 0 & 0 & 0 \\
\hline 291 & 0 & 0 & 0 & 0 & 16989 & 0 & 0 & 0 & 0 & 0 \\
\hline 292 & 0 & 0 & 0 & 0 & 0 & 0 & 0 & 0 & 0 & 0 \\
\hline 293 & 0 & 0 & 0 & 0 & 0 & 0 & 0 & 0 & 0 & 0 \\
\hline 294 & 0 & 0 & 70943 & 0 & 0 & 0 & 0 & 0 & 0 & 0 \\
\hline 295 & 0 & 0 & 0 & 0 & 0 & 0 & 0 & 0 & 0 & 0 \\
\hline 298 & 0 & 17383 & 0 & 0 & 0 & 0 & 0 & 0 & 0 & 0 \\
\hline 301 & 0 & 16188 & 0 & 0 & 0 & 0 & 0 & 0 & 0 & 0 \\
\hline 302 & 0 & 27280 & 0 & 0 & 28693 & 0 & 0 & 0 & 0 & 0 \\
\hline 303 & 0 & 0 & 0 & 0 & 0 & 0 & 0 & 0 & 0 & 0 \\
\hline 304 & 0 & 0 & 0 & 0 & 0 & 0 & 0 & 0 & 0 & 0 \\
\hline 305 & 0 & 20991 & 0 & 0 & 0 & 0 & 0 & 0 & 0 & 0 \\
\hline 306 & 0 & 0 & 0 & 0 & 0 & 0 & 0 & 0 & 0 & 0 \\
\hline 307 & 0 & 0 & 0 & 0 & 0 & 0 & 0 & 0 & 0 & 0 \\
\hline 310 & 0 & 19373 & 0 & 0 & 0 & 0 & 0 & 0 & 0 & 0 \\
\hline 311 & 0 & 31619 & 0 & 0 & 0 & 16505 & 0 & 0 & 0 & 0 \\
\hline 312 & 0 & 0 & 0 & 14808 & 38774 & 0 & 15964 & 0 & 0 & 0 \\
\hline 313 & 0 & 0 & 0 & 0 & 0 & 0 & 16717 & 0 & 0 & 0 \\
\hline 315 & 0 & 0 & 0 & 0 & 0 & 0 & 0 & 0 & 0 & 0 \\
\hline 317 & 0 & 20390 & 0 & 0 & 0 & 0 & 0 & 0 & 0 & 0 \\
\hline 318 & 0 & 0 & 0 & 0 & 0 & 15676 & 0 & 0 & 0 & 0 \\
\hline 320 & 0 & 27308 & 0 & 16809 & 0 & 0 & 0 & 0 & 0 & 0 \\
\hline 322 & 0 & 0 & 0 & 0 & 0 & 0 & 0 & 0 & 0 & 0 \\
\hline 329 & 0 & 0 & 0 & 0 & 0 & 0 & 0 & 0 & 0 & 0 \\
\hline 332 & 23980 & 0 & 0 & 0 & 0 & 0 & 0 & 0 & 0 & 0 \\
\hline 358 & 0 & 0 & 0 & 0 & 0 & 0 & 0 & 0 & 0 & 0 \\
\hline 359 & 0 & 0 & 0 & 0 & 0 & 0 & 0 & 0 & 0 & 0 \\
\hline 362 & 0 & 0 & 0 & 0 & 0 & 0 & 0 & 0 & 0 & 0 \\
\hline
\end{tabular}




\begin{tabular}{|c|c|c|c|c|c|c|c|c|c|c|c|}
\hline 386 & 0 & 0 & 0 & 0 & 0 & 0 & 0 & 0 & 0 & 0 & 0 \\
\hline 402 & 0 & 0 & 0 & 0 & 0 & 0 & 0 & 0 & 0 & 25027 & 26885 \\
\hline 410 & 0 & 0 & 0 & 0 & 0 & 0 & 0 & 0 & 0 & 0 & 0 \\
\hline 430 & 0 & 0 & 0 & 0 & 0 & 0 & 0 & 0 & 0 & 0 & 0 \\
\hline 439 & 0 & 0 & 0 & 0 & 0 & 0 & 0 & 0 & 0 & 0 & 0 \\
\hline 451 & 0 & 0 & 0 & 0 & 0 & 0 & 0 & 0 & 0 & 0 & 0 \\
\hline 459 & 0 & 0 & 0 & 0 & 0 & 0 & 0 & 0 & 0 & 0 & 0 \\
\hline 476 & 0 & 0 & 0 & 0 & 0 & 0 & 0 & 0 & 0 & 0 & 0 \\
\hline 502 & 0 & 0 & 0 & 0 & 0 & 0 & 0 & 0 & 0 & 0 & 0 \\
\hline 503 & 0 & 0 & 0 & 0 & 0 & 0 & 0 & 0 & 0 & 0 & 0 \\
\hline 504 & 0 & 0 & 0 & 0 & 0 & 0 & 0 & 0 & 0 & 0 & 0 \\
\hline 506 & 0 & 0 & 0 & 0 & 0 & 0 & 0 & 0 & 0 & 0 & 0 \\
\hline 507 & 0 & 0 & 0 & 0 & 0 & 0 & 0 & 0 & 0 & 0 & 0 \\
\hline 508 & 0 & 0 & 0 & 0 & 0 & 0 & 0 & 0 & 0 & 0 & 0 \\
\hline 509 & 0 & 0 & 0 & 0 & 0 & 0 & 0 & 0 & 0 & 0 & 0 \\
\hline 511 & 0 & 0 & 0 & 0 & 0 & 0 & 0 & 0 & 0 & 0 & 0 \\
\hline 512 & 0 & 0 & 0 & 0 & 0 & 0 & 0 & 0 & 0 & 0 & 0 \\
\hline 513 & 0 & 0 & 0 & 0 & 0 & 0 & 0 & 0 & 0 & 0 & 0 \\
\hline 515 & 0 & 0 & 0 & 0 & 0 & 0 & 0 & 0 & 0 & 0 & 0 \\
\hline 518 & 0 & 0 & 0 & 0 & 0 & 0 & 0 & 0 & 0 & 0 & 0 \\
\hline 526 & 0 & 0 & 0 & 19371 & 0 & 0 & 0 & 0 & 0 & 0 & 0 \\
\hline 528 & 0 & 0 & 0 & 0 & 0 & 0 & 0 & 0 & 0 & 0 & 0 \\
\hline 530 & 0 & 0 & 0 & 0 & 0 & 0 & 0 & 0 & 0 & 0 & 0 \\
\hline
\end{tabular}




\begin{tabular}{|c|c|c|c|c|c|c|c|c|c|c|c|}
\hline $\mathrm{BP}$ & H-5-205 & H-5-259 & H-5-209 & H-3-201 & H-3-240 & H-3-225 & H-3-243 & H-3-807 & H-3-844 & H-3-183 & $\mathrm{H}-3-193$ \\
\hline 56 & 0 & 0 & 0 & 27445 & 0 & 0 & 0 & 0 & 0 & 37106 & 0 \\
\hline 57 & 0 & 0 & 0 & 0 & 0 & 0 & 0 & 0 & 0 & 27566 & 0 \\
\hline 58 & 0 & 0 & 0 & 0 & 0 & 0 & 0 & 0 & 0 & 0 & 0 \\
\hline 59 & 0 & 36853 & 0 & 0 & 0 & 0 & 0 & 0 & 0 & 0 & 0 \\
\hline 61 & 0 & 0 & 0 & 0 & 0 & 0 & 0 & 0 & 0 & 0 & 0 \\
\hline 62 & 0 & 0 & 0 & 0 & 0 & 0 & 0 & 0 & 0 & 0 & 0 \\
\hline 63 & 0 & 0 & 0 & 0 & 0 & 0 & 0 & 0 & 0 & 0 & 0 \\
\hline 64 & 0 & 41423 & 0 & 0 & 19595 & 0 & 0 & 0 & 0 & 0 & 0 \\
\hline 66 & 0 & 0 & 0 & 0 & 0 & 0 & 0 & 0 & 18377 & 0 & 0 \\
\hline 67 & 0 & 0 & 0 & 0 & 0 & 0 & 0 & 0 & 0 & 0 & 0 \\
\hline 68 & 0 & 31150 & 0 & 38751 & 63221 & 58228 & 47762 & 0 & 18461 & 0 & 27700 \\
\hline 69 & 0 & 0 & 0 & 0 & 0 & 0 & 0 & 0 & 0 & 0 & 0 \\
\hline 70 & 0 & 0 & 0 & 0 & 0 & 0 & 0 & 0 & 64508 & 111134 & 0 \\
\hline 71 & 0 & 25518 & 0 & 0 & 0 & 0 & 0 & 0 & 0 & 0 & 43493 \\
\hline 73 & 0 & 0 & 0 & 0 & 0 & 0 & 0 & 0 & 0 & 0 & 0 \\
\hline 74 & 0 & 21765 & 0 & 0 & 0 & 28250 & 0 & 55451 & 20690 & 12134 & 18852 \\
\hline 75 & 0 & 23071 & 0 & 0 & 0 & 0 & 0 & 0 & 0 & 0 & 0 \\
\hline 76 & 0 & 0 & 0 & 0 & 0 & 0 & 0 & 0 & 0 & 0 & 0 \\
\hline 77 & 0 & 16865 & 0 & 0 & 0 & 0 & 0 & 0 & 0 & 0 & 0 \\
\hline 78 & 0 & 20798 & 0 & 0 & 0 & 0 & 0 & 0 & 25231 & 40293 & 0 \\
\hline 79 & 19050 & 26320 & 0 & 0 & 0 & 0 & 24014 & 0 & 26619 & 12894 & 34480 \\
\hline 81 & 58215 & 50697 & 0 & 21743 & 0 & 18619 & 32353 & 75379 & 41379 & 29529 & 60939 \\
\hline 82 & 0 & 0 & 0 & 0 & 0 & 0 & 0 & 0 & 0 & 0 & 0 \\
\hline 83 & 0 & 15071 & 0 & 0 & 0 & 0 & 0 & 0 & 20690 & 14728 & 0 \\
\hline 84 & 0 & 0 & 0 & 0 & 0 & 0 & 0 & 0 & 0 & 0 & 0 \\
\hline 85 & 0 & 0 & 0 & 0 & 0 & 0 & 0 & 0 & 0 & 0 & 0 \\
\hline 86 & 0 & 0 & 0 & 0 & 0 & 0 & 0 & 0 & 0 & 16006 & 0 \\
\hline 87 & 0 & 0 & 0 & 19134 & 0 & 0 & 0 & 0 & 0 & 0 & 0 \\
\hline 88 & 0 & 0 & 0 & 0 & 0 & 0 & 0 & 0 & 0 & 0 & 0 \\
\hline 89 & 0 & 0 & 0 & 0 & 0 & 0 & 0 & 0 & 0 & 0 & 0 \\
\hline 92 & 0 & 0 & 0 & 0 & 0 & 0 & 0 & 0 & 0 & 0 & 0 \\
\hline
\end{tabular}




\begin{tabular}{|c|c|c|c|c|c|c|c|c|c|c|c|}
\hline 93 & 0 & 25116 & 0 & 52571 & 23414 & 25830 & 33087 & 24976 & 0 & 0 & 0 \\
\hline 94 & 0 & 15331 & 0 & 24063 & 0 & 0 & 0 & 26784 & 0 & 0 & 0 \\
\hline 95 & 0 & 0 & 0 & 19811 & 0 & 0 & 0 & 0 & 17031 & 16784 & 0 \\
\hline 98 & 0 & 20947 & 0 & 33533 & 0 & 0 & 0 & 33753 & 0 & 0 & 0 \\
\hline 99 & 16902 & 23166 & 0 & 0 & 0 & 0 & 0 & 0 & 0 & 0 & 14057 \\
\hline 101 & 42397 & 28869 & 0 & 0 & 0 & 0 & 0 & 0 & 0 & 0 & 0 \\
\hline 102 & 0 & 0 & 0 & 0 & 0 & 0 & 0 & 0 & 0 & 0 & 0 \\
\hline 103 & 20894 & 14812 & 0 & 0 & 0 & 0 & 0 & 0 & 0 & 0 & 0 \\
\hline 104 & 136640 & 57076 & 0 & 0 & 0 & 0 & 0 & 54660 & 28806 & 29659 & 23317 \\
\hline 105 & 0 & 16802 & 0 & 0 & 0 & 0 & 0 & 0 & 0 & 0 & 0 \\
\hline 106 & 23575 & 21765 & 0 & 0 & 0 & 0 & 0 & 0 & 0 & 0 & 0 \\
\hline 107 & 0 & 0 & 0 & 0 & 0 & 0 & 0 & 0 & 0 & 0 & 0 \\
\hline 108 & 0 & 0 & 0 & 0 & 0 & 0 & 0 & 0 & 0 & 0 & 0 \\
\hline 109 & 17491 & 17534 & 0 & 0 & 0 & 0 & 0 & 0 & 0 & 0 & 0 \\
\hline 114 & 0 & 0 & 0 & 0 & 0 & 0 & 0 & 0 & 0 & 0 & 0 \\
\hline 115 & 0 & 0 & 0 & 0 & 0 & 0 & 0 & 0 & 0 & 0 & 0 \\
\hline 116 & 0 & 0 & 0 & 0 & 0 & 0 & 0 & 0 & 0 & 0 & 0 \\
\hline 117 & 18233 & 0 & 0 & 0 & 0 & 0 & 0 & 0 & 0 & 0 & 0 \\
\hline 118 & 0 & 0 & 0 & 0 & 0 & 0 & 0 & 0 & 0 & 0 & 0 \\
\hline 119 & 0 & 0 & 0 & 0 & 0 & 0 & 0 & 0 & 0 & 0 & 0 \\
\hline 120 & 0 & 0 & 0 & 0 & 0 & 0 & 0 & 0 & 0 & 0 & 0 \\
\hline 121 & 0 & 0 & 0 & 0 & 0 & 0 & 0 & 0 & 0 & 0 & 0 \\
\hline 122 & 0 & 0 & 0 & 0 & 0 & 0 & 0 & 0 & 0 & 0 & 0 \\
\hline 124 & 17206 & 0 & 0 & 0 & 0 & 0 & 0 & 0 & 0 & 0 & 0 \\
\hline 126 & 19563 & 0 & 0 & 0 & 0 & 0 & 0 & 0 & 0 & 0 & 0 \\
\hline 127 & 22739 & 0 & 0 & 0 & 0 & 0 & 0 & 0 & 0 & 0 & 0 \\
\hline 128 & 30438 & 21113 & 0 & 19811 & 0 & 20940 & 22880 & 24938 & 0 & 0 & 0 \\
\hline 129 & 20115 & 18477 & 0 & 0 & 0 & 0 & 0 & 0 & 0 & 0 & 0 \\
\hline 130 & 15533 & 0 & 0 & 0 & 0 & 0 & 0 & 0 & 0 & 0 & 22325 \\
\hline 132 & 0 & 0 & 0 & 0 & 0 & 0 & 0 & 0 & 0 & 0 & 0 \\
\hline 134 & 0 & 0 & 0 & 0 & 0 & 0 & 0 & 0 & 0 & 0 & 0 \\
\hline 135 & 0 & 0 & 0 & 0 & 0 & 0 & 0 & 0 & 0 & 0 & 0 \\
\hline 137 & 0 & 0 & 0 & 0 & 0 & 0 & 0 & 0 & 21825 & 0 & 0 \\
\hline
\end{tabular}




\begin{tabular}{|c|c|c|c|c|c|c|c|c|c|c|c|}
\hline 138 & 0 & 0 & 0 & 0 & 0 & 0 & 0 & 0 & 283221 & 630493 & 232843 \\
\hline 139 & 14563 & 0 & 0 & 0 & 0 & 0 & 0 & 0 & 0 & 0 & 0 \\
\hline 142 & 0 & 0 & 0 & 0 & 0 & 0 & 0 & 0 & 0 & 0 & 0 \\
\hline 146 & 27948 & 23158 & 0 & 0 & 0 & 0 & 0 & 0 & 26535 & 0 & 0 \\
\hline 147 & 35629 & 23299 & 0 & 0 & 0 & 0 & 0 & 0 & 23886 & 0 & 0 \\
\hline 149 & 0 & 0 & 0 & 0 & 0 & 0 & 0 & 0 & 0 & 0 & 0 \\
\hline 150 & 61466 & 32094 & 0 & 0 & 18978 & 0 & 0 & 30023 & 22918 & 0 & 0 \\
\hline 151 & 0 & 0 & 0 & 0 & 0 & 0 & 0 & 0 & 0 & 0 & 0 \\
\hline 152 & 0 & 14143 & 0 & 0 & 0 & 0 & 0 & 0 & 0 & 0 & 0 \\
\hline 153 & 0 & 0 & 0 & 0 & 0 & 0 & 0 & 0 & 0 & 0 & 0 \\
\hline 156 & 23841 & 14639 & 0 & 0 & 0 & 0 & 0 & 0 & 0 & 0 & 0 \\
\hline 157 & 0 & 0 & 0 & 0 & 0 & 0 & 0 & 0 & 0 & 0 & 0 \\
\hline 158 & 0 & 0 & 0 & 0 & 0 & 0 & 0 & 0 & 0 & 0 & 0 \\
\hline 160 & 0 & 0 & 0 & 0 & 0 & 0 & 0 & 0 & 0 & 0 & 0 \\
\hline 161 & 0 & 0 & 0 & 0 & 0 & 0 & 0 & 0 & 0 & 0 & 0 \\
\hline 162 & 0 & 0 & 0 & 0 & 0 & 0 & 0 & 0 & 0 & 0 & 0 \\
\hline 165 & 0 & 0 & 0 & 0 & 0 & 0 & 0 & 0 & 0 & 0 & 0 \\
\hline 167 & 0 & 0 & 0 & 0 & 0 & 0 & 0 & 0 & 0 & 0 & 0 \\
\hline 170 & 0 & 0 & 0 & 0 & 0 & 0 & 0 & 0 & 0 & 0 & 0 \\
\hline 171 & 0 & 0 & 0 & 0 & 0 & 0 & 0 & 0 & 0 & 0 & 0 \\
\hline 172 & 0 & 0 & 0 & 0 & 0 & 0 & 0 & 0 & 0 & 0 & 0 \\
\hline 175 & 0 & 0 & 0 & 0 & 0 & 0 & 0 & 0 & 0 & 0 & 0 \\
\hline 176 & 0 & 0 & 0 & 0 & 0 & 0 & 0 & 0 & 0 & 0 & 0 \\
\hline 177 & 0 & 0 & 12007 & 0 & 0 & 0 & 0 & 24825 & 0 & 0 & 0 \\
\hline 178 & 0 & 0 & 15579 & 0 & 0 & 0 & 0 & 0 & 0 & 0 & 0 \\
\hline 179 & 0 & 0 & 16644 & 0 & 0 & 0 & 0 & 0 & 0 & 0 & 0 \\
\hline 180 & 25039 & 19500 & 0 & 0 & 0 & 0 & 0 & 0 & 0 & 0 & 0 \\
\hline 182 & 0 & 0 & 0 & 0 & 0 & 0 & 0 & 0 & 0 & 0 & 0 \\
\hline 184 & 0 & 0 & 0 & 0 & 0 & 0 & 0 & 0 & 0 & 0 & 0 \\
\hline 185 & 0 & 0 & 0 & 0 & 0 & 0 & 0 & 0 & 0 & 0 & 0 \\
\hline 187 & 0 & 0 & 0 & 0 & 0 & 0 & 0 & 0 & 0 & 0 & 0 \\
\hline 188 & 0 & 0 & 0 & 0 & 0 & 0 & 0 & 0 & 0 & 0 & 0 \\
\hline 189 & 16769 & 21435 & 0 & 19231 & 0 & 0 & 0 & 0 & 0 & 0 & 0 \\
\hline
\end{tabular}




\begin{tabular}{|c|c|c|c|c|c|c|c|c|c|c|c|}
\hline 190 & 0 & 0 & 0 & 0 & 0 & 0 & 0 & 0 & 0 & 0 & 0 \\
\hline 191 & 29203 & 23512 & 0 & 0 & 0 & 27460 & 0 & 52287 & 0 & 0 & 0 \\
\hline 192 & 0 & 0 & 0 & 0 & 0 & 0 & 0 & 0 & 0 & 0 & 0 \\
\hline 193 & 25856 & 15842 & 26452 & 0 & 0 & 0 & 0 & 0 & 0 & 0 & 0 \\
\hline 194 & 0 & 0 & 523413 & 0 & 0 & 62821 & 0 & 0 & 0 & 0 & 0 \\
\hline 197 & 30400 & 20711 & 0 & 0 & 27232 & 25089 & 36755 & 24787 & 0 & 0 & 0 \\
\hline 199 & 0 & 0 & 0 & 0 & 0 & 0 & 0 & 24938 & 0 & 0 & 0 \\
\hline 200 & 23727 & 19893 & 0 & 20874 & 0 & 0 & 0 & 0 & 0 & 0 & 0 \\
\hline 201 & 21237 & 16070 & 0 & 66293 & 25535 & 36794 & 43026 & 52513 & 22288 & 0 & 15876 \\
\hline 202 & 54128 & 35657 & 0 & 154716 & 71167 & 103368 & 84918 & 96625 & 61943 & 21675 & 91202 \\
\hline 203 & 35553 & 25777 & 0 & 47835 & 36721 & 38127 & 45294 & 32133 & 0 & 0 & 0 \\
\hline 204 & 0 & 15268 & 0 & 0 & 0 & 0 & 0 & 0 & 0 & 0 & 0 \\
\hline 205 & 0 & 0 & 0 & 0 & 0 & 0 & 0 & 0 & 0 & 0 & 119894 \\
\hline 206 & 0 & 0 & 0 & 0 & 0 & 0 & 0 & 0 & 103616 & 0 & 89549 \\
\hline 210 & 26750 & 23189 & 0 & 0 & 0 & 0 & 0 & 35410 & 0 & 0 & 0 \\
\hline 211 & 54698 & 50398 & 0 & 28701 & 21794 & 34028 & 36822 & 50516 & 0 & 0 & 0 \\
\hline 212 & 0 & 0 & 0 & 0 & 0 & 0 & 0 & 0 & 0 & 0 & 0 \\
\hline 216 & 0 & 0 & 0 & 0 & 0 & 0 & 0 & 0 & 0 & 0 & 0 \\
\hline 217 & 0 & 0 & 0 & 0 & 0 & 55363 & 51631 & 0 & 0 & 0 & 0 \\
\hline 219 & 0 & 0 & 0 & 0 & 0 & 0 & 0 & 25729 & 0 & 0 & 0 \\
\hline 221 & 0 & 0 & 0 & 0 & 0 & 0 & 0 & 0 & 0 & 0 & 0 \\
\hline 222 & 0 & 0 & 0 & 0 & 0 & 0 & 0 & 0 & 0 & 0 & 0 \\
\hline 224 & 0 & 0 & 11758 & 0 & 25419 & 23113 & 22013 & 0 & 0 & 0 & 0 \\
\hline 225 & 0 & 0 & 0 & 0 & 0 & 0 & 0 & 0 & 0 & 0 & 0 \\
\hline 226 & 0 & 0 & 0 & 23386 & 27734 & 0 & 0 & 24410 & 0 & 0 & 0 \\
\hline 227 & 0 & 0 & 18277 & 0 & 38650 & 18718 & 0 & 0 & 0 & 0 & 0 \\
\hline 228 & 0 & 0 & 60115 & 0 & 0 & 18125 & 0 & 0 & 0 & 0 & 0 \\
\hline 229 & 0 & 0 & 64843 & 42424 & 86287 & 33584 & 45294 & 0 & 0 & 0 & 0 \\
\hline 230 & 0 & 0 & 23050 & 32857 & 85632 & 62031 & 65573 & 0 & 0 & 0 & 0 \\
\hline 231 & 0 & 0 & 24411 & 23869 & 43086 & 21385 & 0 & 0 & 0 & 0 & 0 \\
\hline 232 & 0 & 0 & 0 & 25222 & 0 & 0 & 25549 & 0 & 0 & 0 & 0 \\
\hline 233 & 0 & 0 & 73720 & 101566 & 187580 & 60450 & 119672 & 32472 & 0 & 0 & 0 \\
\hline 234 & 14202 & 0 & 0 & 0 & 0 & 0 & 0 & 0 & 0 & 0 & 0 \\
\hline
\end{tabular}




\begin{tabular}{|c|c|c|c|c|c|c|c|c|c|c|c|}
\hline 235 & 0 & 0 & 33119 & 0 & 0 & 0 & 0 & 0 & 0 & 0 & 0 \\
\hline 236 & 0 & 0 & 0 & 0 & 0 & 0 & 0 & 0 & 0 & 0 & 0 \\
\hline 238 & 0 & 0 & 36826 & 0 & 0 & 0 & 0 & 0 & 0 & 0 & 0 \\
\hline 239 & 0 & 0 & 12744 & 0 & 0 & 0 & 0 & 0 & 0 & 0 & 0 \\
\hline 240 & 0 & 0 & 0 & 0 & 0 & 0 & 0 & 0 & 0 & 0 & 0 \\
\hline 241 & 0 & 0 & 0 & 0 & 0 & 0 & 0 & 0 & 0 & 0 & 0 \\
\hline 242 & 0 & 0 & 0 & 0 & 0 & 0 & 0 & 0 & 0 & 0 & 0 \\
\hline 243 & 0 & 0 & 0 & 0 & 0 & 0 & 0 & 0 & 0 & 0 & 0 \\
\hline 244 & 0 & 0 & 0 & 0 & 0 & 0 & 0 & 0 & 0 & 0 & 16702 \\
\hline 250 & 0 & 0 & 0 & 0 & 0 & 0 & 0 & 0 & 0 & 0 & 0 \\
\hline 253 & 0 & 0 & 0 & 0 & 0 & 0 & 0 & 0 & 0 & 0 & 0 \\
\hline 254 & 0 & 0 & 0 & 0 & 0 & 0 & 0 & 0 & 0 & 0 & 0 \\
\hline 255 & 0 & 0 & 0 & 0 & 0 & 0 & 0 & 0 & 0 & 0 & 0 \\
\hline 256 & 0 & 0 & 11440 & 0 & 0 & 0 & 0 & 0 & 0 & 0 & 0 \\
\hline 257 & 0 & 0 & 0 & 0 & 0 & 0 & 35288 & 0 & 0 & 0 & 0 \\
\hline 258 & 0 & 0 & 0 & 0 & 19518 & 23262 & 28350 & 0 & 0 & 0 & 0 \\
\hline 260 & 0 & 0 & 0 & 0 & 0 & 0 & 0 & 0 & 0 & 0 & 0 \\
\hline 261 & 0 & 0 & 0 & 0 & 0 & 0 & 0 & 0 & 0 & 0 & 0 \\
\hline 262 & 0 & 0 & 0 & 0 & 0 & 0 & 0 & 26369 & 0 & 0 & 0 \\
\hline 264 & 0 & 0 & 0 & 0 & 0 & 0 & 0 & 0 & 0 & 0 & 0 \\
\hline 265 & 0 & 0 & 0 & 0 & 0 & 0 & 0 & 0 & 0 & 0 & 0 \\
\hline 266 & 0 & 0 & 0 & 0 & 0 & 0 & 0 & 0 & 0 & 0 & 0 \\
\hline 268 & 0 & 0 & 0 & 0 & 0 & 0 & 0 & 0 & 0 & 0 & 0 \\
\hline 270 & 0 & 0 & 0 & 0 & 0 & 0 & 0 & 0 & 0 & 0 & 0 \\
\hline 272 & 0 & 0 & 0 & 0 & 0 & 0 & 0 & 0 & 0 & 0 & 0 \\
\hline 273 & 0 & 0 & 0 & 0 & 0 & 0 & 0 & 0 & 53953 & 0 & 57549 \\
\hline 274 & 0 & 0 & 0 & 0 & 0 & 0 & 0 & 0 & 0 & 0 & 45229 \\
\hline 275 & 0 & 14875 & 0 & 33630 & 0 & 0 & 0 & 25277 & 0 & 0 & 0 \\
\hline 276 & 0 & 0 & 0 & 0 & 0 & 0 & 0 & 0 & 0 & 0 & 0 \\
\hline 277 & 0 & 0 & 0 & 0 & 0 & 0 & 0 & 0 & 0 & 0 & 0 \\
\hline 278 & 0 & 0 & 0 & 0 & 0 & 0 & 0 & 0 & 0 & 0 & 0 \\
\hline 280 & 0 & 0 & 0 & 0 & 0 & 0 & 0 & 0 & 0 & 0 & 0 \\
\hline 282 & 0 & 0 & 0 & 0 & 0 & 0 & 0 & 0 & 0 & 0 & 0 \\
\hline
\end{tabular}




\begin{tabular}{|c|c|c|c|c|c|c|c|c|c|c|}
\hline 283 & 0 & 0 & 0 & 0 & 49219 & 39115 & 27483 & 0 & 0 & 0 \\
\hline 284 & 0 & 0 & 0 & 0 & 0 & 0 & 0 & 0 & 0 & 0 \\
\hline 285 & 0 & 0 & 0 & 0 & 0 & 0 & 0 & 0 & 0 & 0 \\
\hline 286 & 0 & 0 & 0 & 0 & 0 & 0 & 24882 & 0 & 0 & 0 \\
\hline 289 & 0 & 0 & 0 & 0 & 0 & 0 & 0 & 0 & 0 & 0 \\
\hline 290 & 0 & 0 & 0 & 0 & 0 & 0 & 0 & 0 & 0 & 0 \\
\hline 291 & 0 & 0 & 0 & 0 & 0 & 0 & 0 & 0 & 0 & 0 \\
\hline 292 & 0 & 0 & 0 & 0 & 0 & 0 & 0 & 0 & 0 & 0 \\
\hline 293 & 0 & 0 & 0 & 0 & 0 & 0 & 0 & 0 & 0 & 0 \\
\hline 294 & 0 & 0 & 24286 & 0 & 0 & 23410 & 0 & 0 & 0 & 0 \\
\hline 295 & 0 & 0 & 0 & 0 & 0 & 0 & 0 & 0 & 0 & 0 \\
\hline 298 & 0 & 0 & 0 & 0 & 0 & 0 & 0 & 0 & 0 & 0 \\
\hline 301 & 0 & 0 & 0 & 0 & 0 & 0 & 0 & 0 & 0 & 0 \\
\hline 302 & 0 & 0 & 0 & 0 & 0 & 0 & 0 & 0 & 0 & 0 \\
\hline 303 & 0 & 0 & 0 & 0 & 0 & 0 & 0 & 0 & 0 & 0 \\
\hline 304 & 0 & 0 & 0 & 0 & 0 & 0 & 0 & 0 & 0 & 0 \\
\hline 305 & 0 & 0 & 0 & 0 & 0 & 0 & 0 & 0 & 0 & 0 \\
\hline 306 & 0 & 0 & 0 & 0 & 0 & 0 & 0 & 0 & 0 & 0 \\
\hline 307 & 0 & 0 & 0 & 0 & 0 & 27015 & 24081 & 41626 & 0 & 0 \\
\hline 310 & 0 & 0 & 0 & 0 & 0 & 0 & 0 & 0 & 0 & 0 \\
\hline 311 & 0 & 0 & 0 & 32470 & 44899 & 0 & 26349 & 0 & 0 & 0 \\
\hline 312 & 0 & 0 & 0 & 0 & 39036 & 39313 & 36422 & 0 & 0 & 0 \\
\hline 313 & 0 & 0 & 11315 & 0 & 0 & 0 & 0 & 0 & 0 & 0 \\
\hline 315 & 0 & 0 & 0 & 0 & 0 & 0 & 0 & 0 & 0 & 0 \\
\hline 317 & 0 & 0 & 0 & 0 & 0 & 0 & 0 & 0 & 0 & 0 \\
\hline 318 & 0 & 0 & 0 & 0 & 0 & 0 & 0 & 0 & 0 & 0 \\
\hline 320 & 0 & 0 & 0 & 0 & 0 & 0 & 0 & 0 & 0 & 0 \\
\hline 322 & 0 & 0 & 0 & 0 & 0 & 0 & 0 & 0 & 0 & 0 \\
\hline 329 & 0 & 0 & 0 & 0 & 0 & 0 & 0 & 0 & 0 & 0 \\
\hline 332 & 0 & 0 & 0 & 0 & 0 & 0 & 0 & 0 & 0 & 0 \\
\hline 358 & 0 & 0 & 0 & 20777 & 25767 & 32843 & 26950 & 33866 & 0 & 0 \\
\hline 359 & 0 & 0 & 0 & 21260 & 0 & 0 & 0 & 0 & 0 & 0 \\
\hline 362 & 0 & 0 & 0 & 17974 & 0 & 0 & 0 & 0 & 0 & 0 \\
\hline
\end{tabular}




\begin{tabular}{|c|c|c|c|c|c|c|c|c|c|c|c|}
\hline 386 & 0 & 0 & 0 & 0 & 0 & 0 & 0 & 33188 & 0 & 0 & 0 \\
\hline 402 & 0 & 0 & 0 & 30054 & 19518 & 42720 & 33553 & 37068 & 0 & 0 & 0 \\
\hline 410 & 0 & 0 & 0 & 0 & 0 & 0 & 0 & 0 & 23129 & 0 & 16620 \\
\hline 430 & 0 & 0 & 0 & 0 & 0 & 0 & 0 & 0 & 0 & 0 & 0 \\
\hline 439 & 0 & 0 & 0 & 0 & 0 & 0 & 0 & 0 & 0 & 0 & 0 \\
\hline 451 & 0 & 0 & 0 & 0 & 0 & 0 & 0 & 0 & 0 & 0 & 0 \\
\hline 459 & 0 & 0 & 0 & 0 & 0 & 0 & 0 & 0 & 0 & 0 & 0 \\
\hline 476 & 0 & 0 & 0 & 0 & 0 & 0 & 0 & 0 & 0 & 0 & 0 \\
\hline 502 & 0 & 0 & 0 & 0 & 0 & 0 & 0 & 0 & 0 & 0 & 0 \\
\hline 503 & 0 & 0 & 0 & 0 & 0 & 0 & 0 & 0 & 0 & 0 & 0 \\
\hline 504 & 0 & 0 & 0 & 0 & 0 & 0 & 0 & 0 & 0 & 0 & 0 \\
\hline 506 & 0 & 0 & 0 & 0 & 0 & 0 & 0 & 0 & 0 & 0 & 0 \\
\hline 507 & 0 & 0 & 0 & 0 & 0 & 0 & 0 & 0 & 0 & 0 & 0 \\
\hline 508 & 0 & 0 & 0 & 0 & 0 & 0 & 0 & 0 & 0 & 0 & 0 \\
\hline 509 & 0 & 0 & 0 & 0 & 0 & 0 & 0 & 0 & 0 & 0 & 0 \\
\hline 511 & 0 & 0 & 0 & 0 & 0 & 0 & 0 & 0 & 0 & 0 & 0 \\
\hline 512 & 0 & 0 & 0 & 0 & 0 & 0 & 0 & 0 & 0 & 0 & 0 \\
\hline 513 & 0 & 0 & 0 & 0 & 0 & 0 & 0 & 0 & 0 & 0 & 0 \\
\hline 515 & 0 & 0 & 0 & 0 & 0 & 0 & 0 & 0 & 0 & 0 & 0 \\
\hline 518 & 0 & 0 & 0 & 0 & 0 & 0 & 0 & 0 & 0 & 0 & 0 \\
\hline 526 & 0 & 0 & 0 & 0 & 0 & 0 & 0 & 0 & 0 & 0 & 0 \\
\hline 528 & 0 & 0 & 0 & 0 & 0 & 0 & 0 & 0 & 0 & 0 & 0 \\
\hline 530 & 0 & 0 & 0 & 0 & 0 & 0 & 0 & 0 & 74895 & 0 & 69373 \\
\hline
\end{tabular}

